\title{
Cache Performance for Multimedia Applications
}

Nathan T. Slingerland and Alan Jay Smith

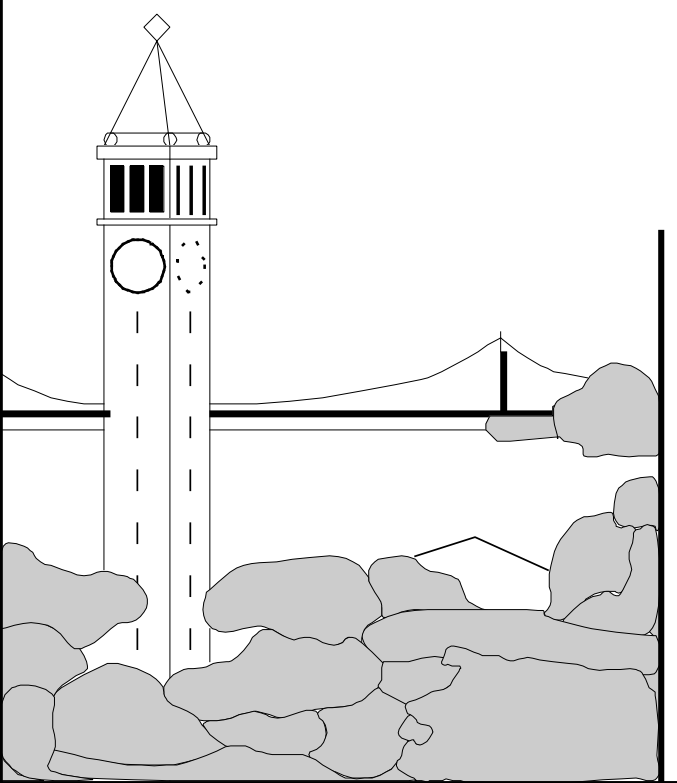

Report No. UCB/CSD-00-1123

December 2000

Computer Science Division (EECS)

University of California

Berkeley, California 94720 


\title{
Cache Performance for Multimedia Applications
}

\author{
Nathan T. Slingerland and Alan Jay Smith \\ \{slingn, smith\}@cs.berkeley.edu \\ Computer Science Division \\ EECS Department \\ University of California, Berkeley
}

December 21, 2000

\begin{abstract}
The caching behavior of multimedia applications has been described as having high instruction reference locality within small loops, very large working sets, and poor data cache performance due to non-locality of data references. Despite this, there is no published research deriving or measuring these qualities. Utilizing the previously developed Berkeley Multimedia Workload, we present the results of execution driven cache simulations with the goal of aiding future media processing architecture design. Our analysis examines the differences between multimedia and traditional applications in cache behavior. We find that multimedia applications actually exhibit lower instruction miss ratios and comparable data miss ratios when contrasted with other widely studied workloads. In addition, we find that longer data cache line sizes than are currently used would benefit multimedia processing.
\end{abstract}

\section{Introduction}

Multimedia is an amalgamation of various data types such as audio, 2D and 3D graphics, animation, images and video within a computing system or within a user application [Bhas97]. Put simply, a multimedia application is one which operates on data to be presented visually or aurally. The purpose of this work is to explore the cache behavior of real world multimedia applications. An important motivation is the widespread belief (seemingly without any actual basis in research) that data caches are not useful for multimedia applications because of the streaming nature of the data upon which they operate [Cont97], [Dief97], [Kuro98], [Lee96], [Rixn98]. The results presented in this paper strongly suggest that contemporary media processing applications perform no worse than traditional integer and floating point workloads.

Funding for this research has been provided by the State of California under the MICRO program, and by Cisco Corporation, Fujitsu Microelectronics, IBM, Intel Corporation, Maxtor Corporation, Microsoft Corporation, Sun Microsystems, Toshiba Corporation and Veritas Software Corporation.

\section{Related Work}

There have been a limited number of multimedia caching studies. [Sode97] studied the data cache behavior of MPEG-2 video decoding with the goal of optimizing playback performance through the cache sensitive handling of the data types used. It was found that although it has been suggested that caches are critically inefficient for video data (several media processor chips dispense with data caches entirely), there was sufficient reuse of values for caching to significantly reduce the raw required memory bandwidth. [Haku97], [Cox98], [Vart98] have studied the usefulness of caching the textures used in 3D rendering. A texture cache with a capacity as small as $16 \mathrm{~KB}$ has been found to reduce the required memory bandwidth three to fifteen times over a non-cached design and exhibit miss ratios around $1 \%$ [Haku97]. The addition of a larger second level of texture cache (2 MB) to a small first level cache (2 KB) can reduce the memory bandwidth from $475 \mathrm{MB} / \mathrm{s}$ to around $92 \mathrm{MB} / \mathrm{s}$ [Cox98].

There have been several studies of prefetching for multimedia. [Zuck96] examined several hardware data prefetching techniques for MPEG-1 (encoding and decoding) and MPEG2 (decoding). Three hardware prefetching techniques were considered, with the most successful found to reduce the miss count by $70 \%$ to $90 \%$. [Stru98] presents a combined hardware/software solution to prefetching for multimedia. Based on cycle accurate simulation of the Trimedia VLIW processor running a highly optimized video de-interlacing application, it was found that such a prefetching scheme was able to eliminate most data cache misses, with the effectiveness dependent on the timing parameters involved. [Cucc99] suggests a two-dimensional prefetching strategy for image data, due to the two separate degrees of spatial locality inherent in image processing (horizontal and vertical). When their 2D prefetching technique was applied to MPEG-2 decoding as well as two imaging applications (convolution and edge tracing), 2D prefetch was found to reduce the miss ratio more than one block look-ahead. Hardware implementation aspects of prefetching are discussed in [Tse98]. 


\section{Multimedia Workload}

\subsection{Description}

For our study of the cache behavior of multimedia applications, we employ the Berkeley Multimedia Workload, which we develop and characterize in [Sling00a]. A description of the component applications and data sets is given in Table 1. The main driving force behind application selection was to strive for completeness in covering as many types of media processing as possible. Open source software was used both for its portability (allowing for cross platform comparisons) as well as the fact that we could directly examine the source code.

The Berkeley workload represents the domains of $3 D$ graphics (Doom, Mesa, POVray), document and image rendering (Ghostscript, DjVu, JPEG), broadband audio (ADPCM, LAME, mpg123, Timidity), speech (Rsynth, GSM, Rasta) and video (MPEG-2). Three MPEG-2 data sets are included to cover Digital Video Disc (DVD) and High Definition Television or HDTV (720P, 1080I) resolutions. The parameters of the DVD, and HDTV data sets are listed in the table below:

\begin{tabular}{|c|c|c|c|c|}
\hline Format & Aspect & Horizontal & Vertical & Frames \\
\hline \hline DVD & $4: 3$ & 720 & 480 & 16 \\
\hline HDTV 720P & $16: 9$ & 1280 & 720 & 16 \\
\hline HDTV 1080I & $16: 9$ & 1920 & 1080 & 16 \\
\hline
\end{tabular}

All of the applications in the Berkeley Multimedia Workload are written in $\mathrm{C}$ with the exception of $\mathrm{Dj} \mathrm{Vu}$ which is coded in $\mathrm{C}++$. Data sets were chosen to be on the order of real workloads, with long enough traces (instruction and data) to exercise very large caches. The trace lengths and other relevant simulation characteristics are listed in Table 2. Total simulation time for our work, not including false starts, machine down time and other simulation problems, was 24.4 days of CPU time for each multimedia run, once with and once without multiprogramming, and 147.2 days of CPU time for SPEC95 simulations, for a grand total of 196 days of CPU time. The machine used was a DEC AlphaStation 255 workstation with a $300 \mathrm{MHz}$ Alpha 21064a).

As it is often necessary to reduce large volues of simulation results into a more easily digestible form, we will use averaging where necessary to compress results. Because the number of applications representing a particular application domain (audio, speech, document, video, 3D) is arbitrary, we will let each of the five application domains comprise a total of $20 \%$ of the averaged workload result, with the component applications of each domain being weighted equally.

\subsection{Motivation}

Motivating our detailed study of the cache behavior of multimedia applications is the large role memory latency plays in limiting performance. The memory hierarchy parameters of bandwidth and latency were measured for several current systems and are shown in Table 3 (the methodology used to obtain these parameters is detailed in the Appendix). Consider Table 4, which compares performance with caching against the same system with all cache levels (L1 and L2) disabled. This was done by setting the appropriate BIOS parameters on our test system at boot time and then measuring the performance on real hardware. From this experiment we can see that modern microprocessor performance is highly dependent on an efficient memory hierarchy. The difference in latency between different levels of contemporary memory hierarchies is substantial, explaining the enormous slowdown we observed when the cache was disabled on our test system. Note that the system time (time spent in the operating system) slowdown is considerably less than that of the user time. This corroborates the generally held belief that the memory locality within operating system code is very poor, as it exhibits less of a performance degradation when caching is disabled.

\begin{tabular}{|c|c|c|}
\hline Name & User Time Ratio & Sys Time Ratio \\
\hline ADPCM Encode & 25.0 & 3.6 \\
\hline ADPCM Decode & 32.9 & 11.3 \\
\hline DJVU Encode & 56.7 & 3.6 \\
\hline DJVU Decode & 61.0 & 16.8 \\
\hline Doom & 53.0 & 1.4 \\
\hline Ghostscript & 63.7 & 34.7 \\
\hline GSM Encode & 61.3 & 6.7 \\
\hline GSM Decode & 77.8 & 16.5 \\
\hline JPEG Encode & 103.4 & 1.3 \\
\hline JPEG Decode & 103.0 & 10.5 \\
\hline LAME & 80.3 & 1.4 \\
\hline Mesa Gears & 44.2 & 11.0 \\
\hline Mesa Morph3D & 35.9 & 35.0 \\
\hline Mesa Reflect & 77.4 & 17.5 \\
\hline MPEG-2 Encode DVD & 86.3 & 2.3 \\
\hline MPEG-2 Encode $720 P$ & 82.9 & 1.4 \\
\hline MPEG-2 Encode 1080I & 86.5 & 1.5 \\
\hline MPEG-2 Decode DVD & 94.1 & 9.3 \\
\hline MPEG-2 Decode $720 P$ & 95.1 & 5.9 \\
\hline MPEG-2 Decode 1080I & 91.9 & 10.4 \\
\hline $\operatorname{mpg} 123$ & 83.7 & 7.5 \\
\hline POVray3 & 74.5 & 16.0 \\
\hline Rasta & 83.8 & 7.0 \\
\hline Rsynth & 86.5 & 27.0 \\
\hline Timidity & 73.9 & 20.3 \\
\hline Arithmetic Mean & 72.6 & 11.2 \\
\hline Geometric Mean & 68.6 & 7.1 \\
\hline
\end{tabular}

Table 4: Uncached Performance Slowdown Factor ( $\left.t_{\text {uncached }} / t_{\text {cached }}\right)$ when L1 and L2 caches were disabled on a $500 \mathrm{MHz}$ AMD Athlon, 64 Kbyte L1 data cache, 64 Kbyte L1 instruction cache, 512 Kbyte L2 unified cache, 64-byte line size.

As another way to see the importance that caches play in modern computer performance, the actual measured total user time for each application was compared with the optimal user run time (a computed result based on the number and type of instructions executed) reported by the DEC ATOM pixie tool, which does not include memory latency and other effects in its measurements. Thus, the pixie results represent 


\begin{tabular}{|c|c|c|}
\hline Name & Description & Data Set \\
\hline $\mathrm{ADPCM}$ & IMA ADPCM audio compression & Excerpt from Shchedrin's Carmen Suite, 28 sec., Mono, 16-bits, $44 \mathrm{kHz}$ \\
\hline $\mathrm{Dj} V u$ & AT\&T IW44 wavelet image compression & $491 \times 726$ color digital photographic image \\
\hline Doom & Classic first person shooter video game & 25.8 sec. recorded game sequence ( 774 frames @ $30 \mathrm{fps}$ ) \\
\hline Ghostscript & Postscript document viewing/rendering & First page of Rosenblum and Ousterhout's LFS paper (24.8 KB) \\
\hline GSM & European GSM 06.10 speech compression & Speech by U.S. Vice President Gore, 24 sec., Mono, 16-bits, 8 kHz \\
\hline JPEG & DCT based lossy image compression & $491 \times 726$ color digital photographic image \\
\hline LAME & MPEG-1 Layer III (MP3) audio encoder & Excerpt from Shchedrin's Carmen Suite, 28 sec., Stereo, 16 -bits, $44 \mathrm{kHz}$ \\
\hline Mesa & OpenGL 3D rendering API clone & Animated gears, morph3d, reflect demos - 30 frames each at $1024 \times 768$ \\
\hline MPEG-2 & MPEG-2 video encoding & 16 frames $(1 \mathrm{GOP})$ at DVD, HDTV $720 \mathrm{P}$, HDTV $1080 \mathrm{I}$ resolutions \\
\hline mpg123 & MPEG-1 Layer III (MP3) audio decoder & Excerpt from Shchedrin's Carmen Suite, 28 sec., Stereo, 16 -bits, $44 \mathrm{kHz}$ \\
\hline POVray & Persistance of Vision ray tracer & 640x480 Ammonite scene by artist Robert A. Mickelson \\
\hline Rasta & Speech recognition & 2.128 sec. SPHERE audio file: "Laurie?...Yeah...Oh." \\
\hline Rsynth & Klatt speech synthesizer & 181 word excerpt of U.S. Declaration of Independence ( 90 sec., 1,062 bytes $)$ \\
\hline Timidity & MIDI music rendering with GUS instruments & X-files theme song, MIDI file ( 49 sec., 13,894 bytes), Goemon patch kit \\
\hline
\end{tabular}

Table 1: Berkeley Multimedia Workload

the best possible execution time for a given sequence of instructions. We assume that memory latency is the dominant factor for why measured performance does not match the theoretically optimal performance. The comparison is shown for DEC AlphaStation 255 workstations with a $300 \mathrm{MHz}$ Alpha 21064a processor and $128 \mathrm{MB}$ of RAM in Table 5.

\section{Methodology}

In order to measure cache miss ratios, we modified the LibCheetah v2.1 implementation [Aust] of the trace driven Cheetah cache simulator [Sugu93] to operate in an execution driven mode. It was also extended to allow for traces longer than $2^{31}$ references long. Cheetah simultaneously evaluates many alternative uniprocessor caches, but restricts the design options that can be varied. For each pass through an address trace, all of the caches evaluated must have the same block size, do no prefetching, and use the LRU or MIN replacement algorithms. Other cache simulators were also considered for this study (TychoII [WARTS], Dinero IV [Edler]), but were found to be considerably slower than Cheetah or otherwise unsuitable for use in execution driven simulation due to dynamic memory allocation issues. (See [Uhli97] and [Smit94] for overviews of trace driven simulation in general, and [Roth99b] for a comparison of the performance of a variety of execution and trace driven solutions.) DEC's ATOM [DEC] was used to instrument target applications with the modified Cheetah simulator, allowing for execution driven cache simulation.

\subsection{Trace Length}

Many cache studies utilize trace lengths that are a fraction of an application's total run time due the enormous simulation times required to account for every instruction and data cache reference. Unfortunately, short trace lengths are problematic because programs exhibit phase behavior; an effect which is easily seen in Figure 1.

\begin{tabular}{|c|c|c|c|}
\hline Name & $\begin{array}{r}\text { Measured } \\
(\mathrm{sec})\end{array}$ & $\begin{array}{r}\text { Optimal } \\
(\mathrm{sec})\end{array}$ & $\begin{array}{r}\text { Percent } \\
\text { Difference }\end{array}$ \\
\hline ADPCM Encode & 0.324 & 0.260 & $19.85 \%$ \\
\hline ADPCM Decode & 0.225 & 0.162 & $27.82 \%$ \\
\hline DJVU Encode & 2.482 & 1.592 & $35.86 \%$ \\
\hline DJVU Decode & 1.848 & 1.164 & $37.01 \%$ \\
\hline Doom & 9.150 & 7.605 & $16.89 \%$ \\
\hline Ghostscript & 6.370 & 3.454 & $45.78 \%$ \\
\hline GSM Encode & 2.511 & 2.375 & $5.42 \%$ \\
\hline GSM Decode & 0.739 & 0.717 & $2.98 \%$ \\
\hline JPEG Encode & 1.046 & 0.873 & $16.56 \%$ \\
\hline JPEG Decode & 0.537 & 0.433 & $19.33 \%$ \\
\hline LAME & 71.300 & 40.680 & $42.95 \%$ \\
\hline Mesa Gears & 3.450 & 1.152 & $66.61 \%$ \\
\hline Mesa Morph3D & 2.950 & 0.998 & $66.18 \%$ \\
\hline Mesa Reflect & 17.660 & 13.970 & $20.89 \%$ \\
\hline MPEG-2 Encode DVD & 74.428 & 64.630 & $13.16 \%$ \\
\hline MPEG-2 Encode $720 \mathrm{P}$ & 201.663 & 172.500 & $14.46 \%$ \\
\hline MPEG-2 Encode 1080I & 460.429 & 397.700 & $13.62 \%$ \\
\hline MPEG-2 Decode DVD & 5.760 & 4.971 & $13.70 \%$ \\
\hline MPEG-2 Decode 720P & 17.980 & 15.280 & $15.02 \%$ \\
\hline MPEG-2 Decode 1080I & 36.230 & 30.360 & $16.20 \%$ \\
\hline mpg123 & 3.830 & 2.620 & $31.59 \%$ \\
\hline POVray3 & 66.442 & 32.780 & $50.66 \%$ \\
\hline Rasta & 0.183 & 0.112 & $39.02 \%$ \\
\hline Rsynth & 2.282 & 2.005 & $12.14 \%$ \\
\hline Timidity & 35.223 & 32.150 & $8.72 \%$ \\
\hline \multicolumn{3}{|c|}{ Arithmetic Mean } & $26.10 \%$ \\
\hline \multicolumn{3}{|c|}{ Geometric Mean } & $20.55 \%$ \\
\hline
\end{tabular}

Table 5: Effect of Memory Latency on Execution Time - DEC AlphaStation 255 with $300 \mathrm{MHz}$ Alpha 21064a processor, 16 Kbyte L1 instruction cache, 16 Kbyte L1 data cache, 1 Mbyte L2 unified cache

The graphs depict the number of cache misses per 1,000,000 instructions executed for two sample applications. A second 


\begin{tabular}{|c|c|c|c|c|c|c|c|c|}
\hline Name & $\begin{array}{r}\text { Instruction } \\
\text { References }\end{array}$ & $\begin{array}{r}\text { Load } \\
\text { References }\end{array}$ & $\begin{array}{r}\text { Store } \\
\text { References }\end{array}$ & $\begin{array}{r}\text { Cache Purge } \\
\text { Interval }\end{array}$ & $\begin{array}{l}\text { Data } \\
\text { Time }\end{array}$ & $\begin{array}{l}\text { User } \\
\text { Time }\end{array}$ & $\begin{array}{r}\text { System } \\
\text { Time }\end{array}$ & $\begin{array}{r}\text { Max Resident } \\
\text { Set Size (kB) }\end{array}$ \\
\hline ADPCM Enc. & $64,020,339$ & $4,302,782$ & 616,116 & 708,037 & 27.818 & 0.102 & 0.036 & 1,472 \\
\hline ADPCM Dec. & $49,687,192$ & $4,302,782$ & $1,229,491$ & 708,037 & 27.818 & 0.054 & 0.067 & 1,472 \\
\hline DJVU Enc. & $394,242,073$ & $68,204,647$ & $27,458,767$ & $4,754,521$ & - & 0.700 & 0.033 & 41,664 \\
\hline DJVU Dec. & $328,761,829$ & $59,700,283$ & $31,845,270$ & $4,754,521$ & - & 0.484 & 0.037 & 20,992 \\
\hline Doom & $1,889,897,116$ & $500,225,773$ & $109,222,846$ & $4,284,671$ & 25.800 & 2.216 & 0.939 & 26,432 \\
\hline Ghostscript* & $970,395,449$ & $188,116,952$ & $96,837,718$ & $1,227,194$ & - & 1.190 & 0.164 & 32,192 \\
\hline GSM Enc. & $375,971,389$ & $55,009,077$ & $14,010,892$ & 297,641 & 24.341 & 0.468 & 0.016 & 1,024 \\
\hline GSM Dec. & $126,489,950$ & $10,711,683$ & $3,812,483$ & 297,641 & 24.341 & 0.209 & 0.014 & 1,024 \\
\hline JPEG Enc. & $177,977,854$ & $41,182,069$ & $14,156,413$ & $3,821,284$ & - & 0.223 & 0.006 & 10,880 \\
\hline JPEG Dec. & $80,176,365$ & $16,419,065$ & $4,585,079$ & $3,821,284$ & - & 0.093 & 0.024 & 10,880 \\
\hline LAME* & $7,989,818,554$ & $1,688,230,256$ & $720,826,607$ & $3,358,692$ & 27.818 & 18.543 & 0.075 & 7,104 \\
\hline Mesa Gears* & $296,287,705$ & $36,839,087$ & $38,449,257$ & $2,173,610$ & 1.000 & 0.484 & 0.039 & 50,240 \\
\hline Mesa Morph3D* & $239,456,087$ & $28,181,931$ & $42,865,365$ & $2,173,610$ & 1.000 & 0.467 & 0.050 & 50,432 \\
\hline Mesa Reflect* & $2,752,665,912$ & $431,196,702$ & $221,523,544$ & $2,173,610$ & 1.000 & 3.672 & 0.051 & 59,968 \\
\hline MPEG2 Enc. DVD & $17,986,999,069$ & $3,257,725,765$ & $554,222,287$ & $5,339,432$ & 0.533 & 17.896 & 0.199 & 48,128 \\
\hline MPEG2 Enc. 720P & $47,606,551,352$ & $8,581,717,942$ & $1,563,082,541$ & $5,339,432$ & 0.533 & 48.263 & 0.505 & 124,032 \\
\hline MPEG2 Enc. 1080I & $111,041,463,652$ & $20,148,301,625$ & $3,349,482,784$ & $5,339,432$ & 0.533 & 113.050 & 0.521 & 277,952 \\
\hline MPEG2 Dec. DVD & $1,307,000,398$ & $219,595,775$ & $76,688,056$ & $1,055,372$ & 0.533 & 1.911 & 0.051 & 16,512 \\
\hline MPEG2 Dec. $720 \mathrm{P}$ & $3,992,213,571$ & $673,343,544$ & $243,881,680$ & $1,055,372$ & 0.533 & 5.796 & 0.141 & 41,472 \\
\hline MPEG2 Dec. 1080I & $8,038,214,930$ & $1,341,912,185$ & $464,649,094$ & $1,055,372$ & 0.533 & 12.098 & 0.182 & 91,648 \\
\hline $\operatorname{mpg} 123^{*}$ & $574,034,774$ & $166,675,525$ & $45,334,678$ & $1,554,505$ & 27.818 & 0.735 & 0.015 & 3,328 \\
\hline POVray3 & $6,017,197,975$ & $1,562,189,592$ & $683,690,648$ & $5,928,433$ & 0.033 & 11.296 & 0.121 & 16,000 \\
\hline Rasta* & $25,120,492$ & $5,925,648$ & $1,989,604$ & $2,560,537$ & 2.128 & 0.039 & 0.014 & 5,632 \\
\hline Rsynth & $402,500,964$ & $102,351,142$ & $39,223,906$ & 594,438 & 99.680 & 0.780 & 0.004 & 7,808 \\
\hline Timidity & $4,588,632,916$ & $1,340,471,112$ & $594,047,710$ & $3,675,086$ & 47.440 & 2.036 & 0.104 & 25,664 \\
\hline Total & $217,315,777,907$ & $40,532,832,944$ & $8,943,732,836$ & - & - & 242.805 & 3.406 & - \\
\hline Arithmetic Mean & $8,692,631,116$ & $1,621,313,318$ & $357,749,313$ & - & - & 9.712 & 0.136 & - \\
\hline
\end{tabular}

Table 2: Berkeley Multimedia Workload Simulation Characteristics - data time (inherent time represented in data set - machine independent), user time (time spent processing in user space - machine dependent), and system time (time spent processing in system space on behalf of an application - machine dependent) in seconds. The cache purge interval is the number of instructions executed in each context interval before flushing the simulated cache. Resident Set is the maximum number of kilobytes in memory active at any one time, as determined by the getrusage() system call. All measurements were done on a DEC Alpha DS20 workstation with dual $500 \mathrm{MHz}$ Alpha 21264 processors and $2048 \mathrm{MB}$ of RAM running Compaq Tru64 Unix v5.0A (Rev. 1094). All applications were compiled with GCC v2.8.1 except $\left(^{*}\right)$ compiled with DEC C v5.6-075.

\begin{tabular}{|c|c|c|c|c|c|c|}
\hline System & L1 $t_{\text {latency }}$ & L1 $r_{x f e r}$ & L2 $t_{\text {latency }}$ & L2 $r_{x f e r}$ & Main Memt $t_{\text {latency }}$ & Main Mem $r_{x f e r}$ \\
\hline \hline Microstar* AMD Athlon $(500 \mathrm{MHz})$ & $4.0 \mathrm{~ns}$ & $2657.18 \mathrm{MB} / \mathrm{s}$ & $109.7 \mathrm{~ns}$ & $1182.90 \mathrm{MB} / \mathrm{s}$ & $242.5 \mathrm{~ns}$ & $305.76 \mathrm{MB} / \mathrm{s}$ \\
\hline DEC DS10 Alpha $21264(466 \mathrm{MHz})$ & $4.3 \mathrm{~ns}$ & $1939.14 \mathrm{MB} / \mathrm{s}$ & $30.4 \mathrm{~ns}$ & $825.27 \mathrm{MB} / \mathrm{s}$ & $197.2 \mathrm{~ns}$ & $336.92 \mathrm{MB} / \mathrm{s}$ \\
\hline BX** Intel Pentium III $(450 \mathrm{MHz})$ & $4.4 \mathrm{~ns}$ & $1695.97 \mathrm{MB} / \mathrm{s}$ & $46.6 \mathrm{~ns}$ & $806.94 \mathrm{MB} / \mathrm{s}$ & $149.8 \mathrm{~ns}$ & $308.33 \mathrm{MB} / \mathrm{s}$ \\
\hline HP N-Class PA-8500 $(3 \times 450 \mathrm{MHz})$ & $4.6 \mathrm{~ns}$ & $2190.42 \mathrm{MB} / \mathrm{s}$ & - & - & $293.3 \mathrm{~ns}$ & $338.50 \mathrm{MB} / \mathrm{s}$ \\
\hline
\end{tabular}

Table 3: Memory Latency and Bandwidth - where $t_{\text {latency }}$ is the time delay for any memory transaction, consisting primarily of memory latency and address transmission time and $r_{x f e r}$ is the bus transfer rate or bandwidth in bytes transferred per unit time. (*)Microstar - Microstar 6167 motherboard utilizing AMD's AMD-750 chipset, Mandrake Linux v7.0, 256 MB RAM $\left.{ }^{* *}\right) \mathrm{BX}$ - unknown motherboard employing the Intel 440BX chipset, RedHat Linux v6.0, 128 MB RAM

difficulty with short trace lengths specific to cache simulations is the cold start problem. Cache simulation programs typically start with an empty cache which becomes filled as the simulation progresses. All initial memory accesses will miss the cache (compulsory misses), so cold start effects can potentially dominate if traces are too short to mitigate these effects.
Traces of a billion or more references may be needed to fully initialize multi-megabyte cache configurations [Kess91].

In order to be able to simulate the effects of a program's behavior, it is necessary to have a trace which captures all of its behavior. We found that although there are some applications (notably many of the SPEC92/95 benchmarks) that exhibit 

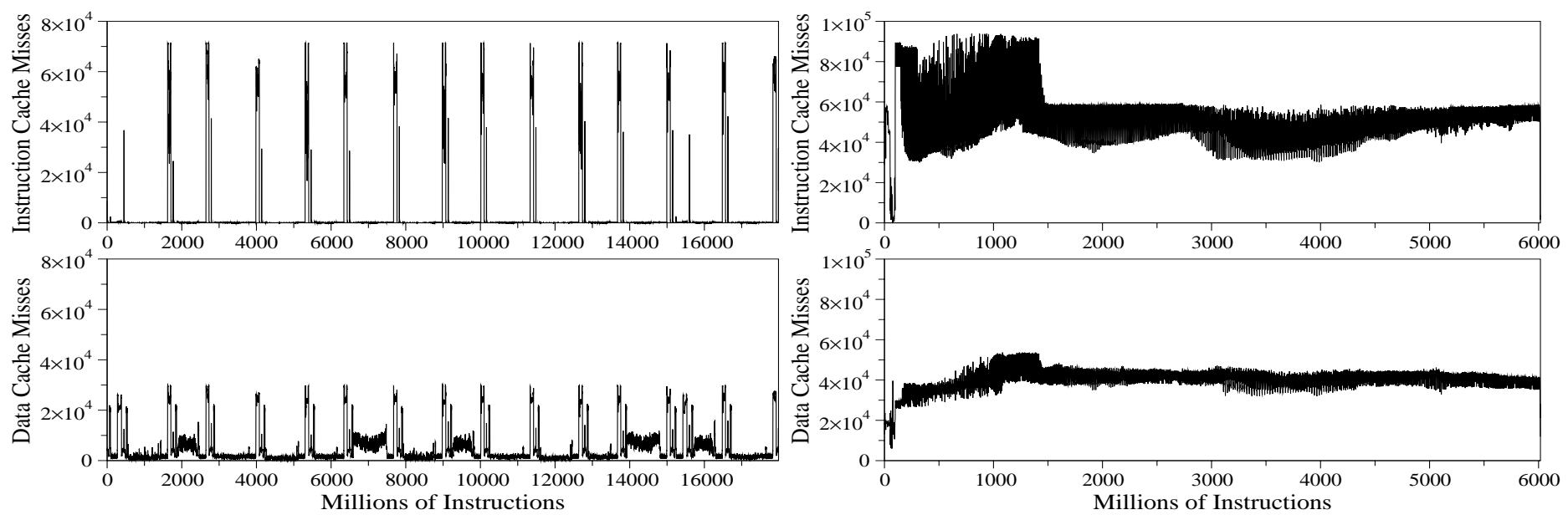

(a) MPEG-2 Encode

(b) POVray

Figure 1: Example Cache Miss Profiles - the number of data cache misses per million instructions executed (direct mapped, 8 Kbyte cache with 32 byte line size)

uniform cache behavior over their entire run times, our multimedia workload applications did not share this property. The result of this is that full applications traces are the only way to completely characterize cache behavior. Our work traces application programs with realistic data sets for full execution runs. The trace lengths for the component Berkeley Multimedia Workload applications are given in Table 2.

\subsection{Operating System Behavior}

In general, studies including operating system behavior are rare because of the difficulty involved in obtaining this information. User space is freely manipulated, but tracing system space usually requires that modifications be made to the operating system. Table 2 lists the amount of time the CPU spends either in user space (user time) doing actual work for the application, or in system space (system time) serving I/O requests and dealing with other overhead on behalf of the application. Both user time and system time are machine dependent, and vary based on the instruction set, clock cycle length and other architectural parameters. Data time is machine independent, and is the implicit time length of the data set. For example, 24 frames of DVD movie represent one second of data time, even though it may take only 0.5 seconds of actual computation time (the sum of system and user time) to process the data. Although our traces only include user state references, this represents almost all of the memory behavior of the programs under study; less than $1 \%$ of our multimedia workload was system time. To some degree this may be an artifact of the nature of the Berkeley Multimedia Workload, which involves only file I/O. In an actual multimedia application where data must be transferred to and from $\mathrm{I} / \mathrm{O}$ devices such as network, disk, or sound and video controller cards, a larger amount of OS activity could be present.

\subsection{Multiprogramming}

Despite the fact that the Berkeley Multimedia Workload is dominated by user time computation, because of multiprogramming we cannot ignore operating system behavior. Although a quantum length that depends on clock time or external events remains constant with architectural change (10 to $100 \mathrm{~ms}$ ), the number of cycles $Q$ in each quantum has increased over time for various reasons, including less efficient software and a speedup of the processor relative to the speed of real time events. The number of cycles in a quantum affects cache miss ratio. When a context switch occurs, the instructions and data of the newly scheduled process may no longer be in the cache from the last time it was run due to the memory use of programs scheduled in the interim.

The effect of multiprogramming can be roughly approximated by periodically flushing (clearing) a simulated cache. In order to correctly simulate the effect of multiprogramming for our multimedia workload, the average context switching interval for commercial (closed source) Microsoft Windows applications was measured on real hardware. The applications were chosen to correspond as closely as possible to those comprising the Berkeley Multimedia Workload, such that, for example, the context interval measured for actual DVD video playback was used in our simulations of MPEG-2 video decoding at DVD resolutions. The context switch intervals of the actual applications from the Berkeley Multimedia Workload were not measured because they are primarily file based applications, typically converting between compressed and uncompressed format without presenting the resulting data to the user. So, although the algorithms they employ (and therefore their memory access patterns) should for the most part be realistically similar to their commercial counterparts, their scheduling behavior is vastly different. Microsoft Windows NT and Windows 2000 both maintain a large amount of performance information for a large number of system objects 
including context switch count, user time and system time per thread. By dividing the sum of system time and user time by the measured context switch count it was possible to compute the average context switch interval for each type of application. The details of these measurements are given in the Appendix, with the simulation quanta (cache flush intervals) applied to each application listed in Table 2.

For comparison purposes, the SPEC95 benchmark suite was also simulated. SPEC95 was simulated without multiprogramming (cache flushing) for several reasons. First, it is normally run in a uniprogrammed mode in order to extract the highest benchmark performance [Gee93]. More importantly, when we measured the actual context switch intervals for SPEC95 on a modern DEC Alpha workstation (DS20 with dual $500 \mathrm{MHz}$ Alpha 21264 processors), the context interval, $Q$, was measured to be sufficiently large such (2.2 million instructions, on average) that multiprogramming has very little effect on miss ratios (unlike multimedia applications which typically have several tightly cooperating threads, the SPEC applications are single threaded and entirely compute bound).

Many UNIX-type operating systems maintain context switch counts on a per process basis which is accessible through the getrusage() system call. The average context switch interval was computed in the same manner as for the Windows multimedia applications. The measured context switch intervals for SPEC95 are presented in the Appendix.

The component applications for both the multimedia workload and SPEC95 were compiled for the Alpha AXP architecture running Digital UNIX v4.0E with the default optimization levels in the case of the multimedia workload, and the base optimization level for SPEC95 (the same compiler optimization flags on all applications: -fast -05 -non_shared). The resulting binaries were then instrumented with the Cheetah cache simulator using ATOM and run on $300 \mathrm{MHz}$ DEC Alpha AXP machines with $128 \mathrm{MB}$ of RAM.

\section{Simulation Results}

The two major determinants of cache performance are $a c$ cess time (the latency from the beginning of an access until the time the requested data is retrieved) and miss ratio (the fraction of cache references which are not found in the cache) [Smit82]. Based on the latencies of a particular cache memory candidate design, in combination with the simulated or measured miss ratio, it is possible to select the design with the highest overall performance (lowest average memory access time) at some level of implementation cost.

Complete tables of the results from all of our simulations are available on the world wide web at http://www.cs.berkeley.edu/〜slingn/research/, from which the cache performance of any application set of interest can be computed. For the sake of brevity, only overall average miss ratios are included in the Appendix.

\subsection{Capacity}

Capacity, or total cache size, has the greatest effect on miss ratio, and so it is one of the most important cache design parameters. Capacity, especially for L1 caches which are typically on the same die as the CPU, is limited by physical die size and implementation cost. In addition, the larger the capacity of a cache, the slower it is due to increased loading of critical address and data lines, thus requiring additional buffering [Przy88]. In order to study the effect of cache capacity on miss ratio, caches were simulated ranging in size from $1 \mathrm{~K}$ to $2 \mathrm{M}$ bytes.

\subsubsection{Other Workloads}

The results of other studies, with a variety of workloads, on the effect of cache size on the miss ratio are presented alongside our simulation results for the Berkeley Multimedia Workload. All of the miss ratios presented in Figures 2, 3, and 4 are for caches with a line size of 32 bytes and two-way associativity, which represent common values for these parameters in current cache systems. Because the results shown have been gathered from a motley assortment of studies of disparate ages and architectures, many of which did not analyze configurations precisely identical to ours in terms of line size and associativity, we use adjusted results from [Gee93]. These adjustments modify the original results of the studies according to the ratios of miss ratios found in [Hill89] for differences in associativity, and [Smit87] for variations in line size. Extensions to larger cache sizes were made for the DTMR results using the $\sqrt{2}$ rule from [Smit82]. Each of the workloads is described in detail in the Appendix. It is important to note that many of the other studies included for comparison purposes also measured or simulated multiprogramming behavior, but because they are based on older machine architectures, their $Q$ (quantum) lengths and therefore their context switch intervals are significantly shorter than those used in our simulations.

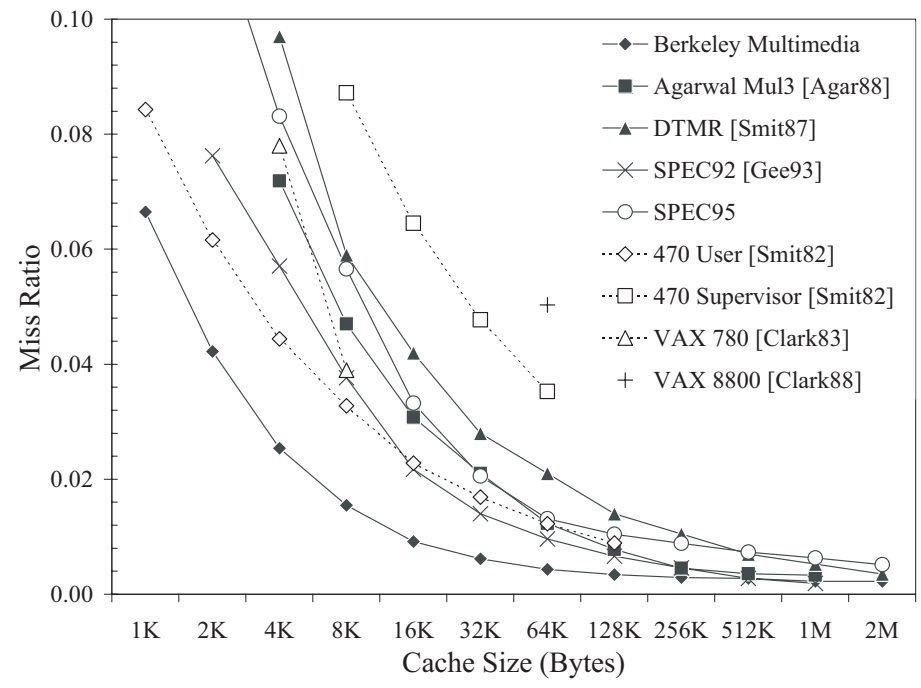

Figure 2: Unified Cache Miss Ratio - 32 byte line size 


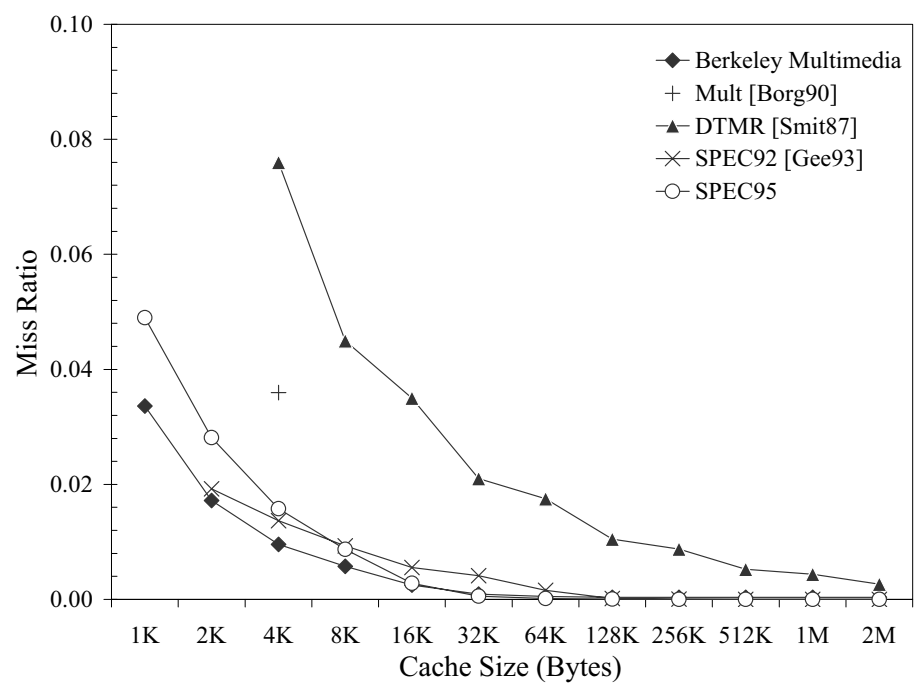

Figure 3: Instruction Cache Miss Ratio - 32 byte line size

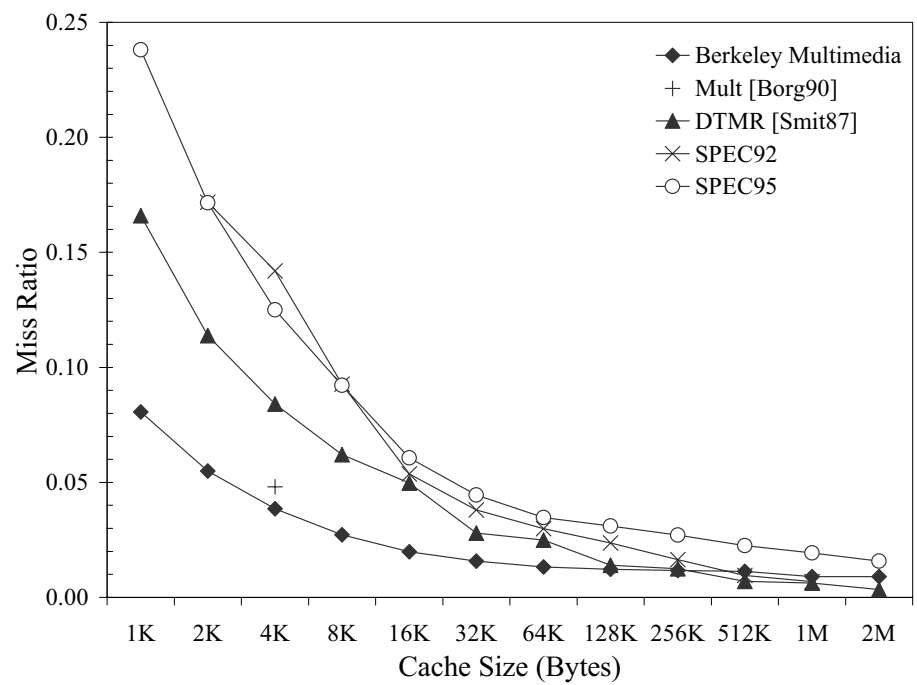

Figure 4: Data Cache Miss Ratio - 32 byte line size

The most significant result of Figures 2, 3, and 4 is that far from multimedia applications exhibiting degenerate cache behavior in comparison to more traditional workloads, our results demonstrate that they actually perform better for nearly all examined cache configurations. We believe that this is attributable to several factors. First, most of the comparison workloads are for timeshared machines on which task switching between users occurred very frequently. Further, the comparison studies are of architectures with much lower clock speeds than modern processors, and so exhibit higher miss ratios due to shorter context switch intervals based on real time periods. Even so, the uniprogrammed SPEC92 and SPEC95 benchmarks still demonstrate higher miss ratios than our multimedia workload. We believe that this is because many multimedia algorithm building blocks (such as the discrete cosine transform and fast Fourier transform) internally reference the same data locations repeatedly. In the case of streaming multimedia applications, data is typically copied into a fixed region of memory (buffer) from the source file of network interface device. Even algorithms which simply traverse enormous arrays of data without re-referencing (color space conversion, subsampling) typically do so in linear memory order, and so benefit greatly from the "prefetching" effect of long cache lines. In addition, multimedia data types are typically small (8-bits for video and speech, 16-bits for audio, single precision (32-bit) floating point for 3D geometry calculations). This means that in comparison to the other workloads which utilize full 32-bit integers or 64-bit (double precision) floating point, more multimedia data elements fit in a single cache line, thus improving the relative hit ratio. Finally, it is also possible that the memory behavior of the Berkeley Multimedia Workload component applications have had their memory behavior tuned in order to avoid cache problems.

\subsubsection{Multimedia Domains}

When broken down into the five application domains (audio, speech, document, video and 3D graphics), some important trends become apparent (Figure 5). Instruction cache miss ratios are quite similar across the various application domains, with a $16 \mathrm{~KB}$ or $32 \mathrm{~KB}$ cache being sufficient. This supports the idea that multimedia applications are dominated by small kernel loops, rather than large code sizes. Data cache miss ratios show significant variation between domains. Speech, video, and audio domains exhibit similar (low miss ratio) cache performance, while the document and $3 \mathrm{D}$ applications have higher miss ratios. This is attributable to the non-linear way in which data sets are traversed during processing for these applications.

\subsubsection{SIMD Effects}

The motivation behind SIMD within a register approach taken by multimedia extensions such as Intel's MMX or Motorola's AltiVec is the fact that on general purpose microprocessors data paths are typically 32 or 64 -bits wide, while multimedia applications typically deal with narrower width data. By packing multiple narrow operations into the wider native processor data path, it is possible to improve multimedia performance.

Although it might be expected that current scalar compilers would place multiple short values into a register and then extract them with register to register operations in order to minimize memory access overhead, we found that this was not the case for the compilers available on our DEC Alpha test platform. Instead, multiple independent short loads are issued. Because of this, the use of SIMD instruction set extensions for multimedia will result in higher cache miss ratios, although the total number of memory references would decrease, due to the folding of several scalar load operations into a single parallel operation for sub-word data types which are adjacent in memory. Note that programs employing multimedia (SIMD) instruction sets are likely to be handcoded, as no currently available commercial compilers are able to gen- 


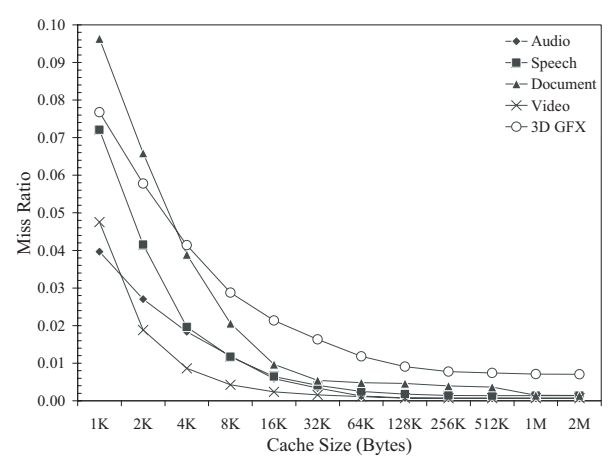

(a) Unified Cache

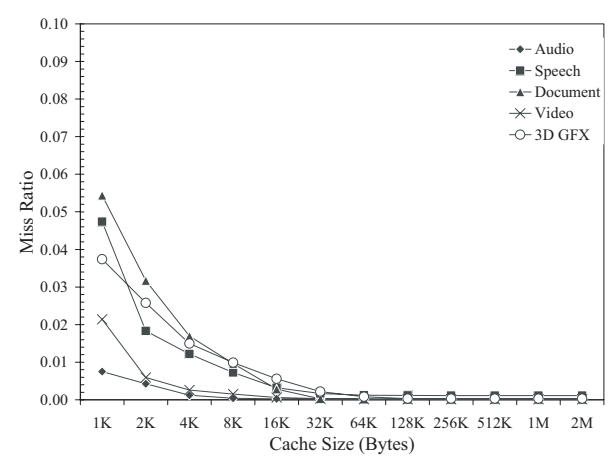

(b) Instruction Cache

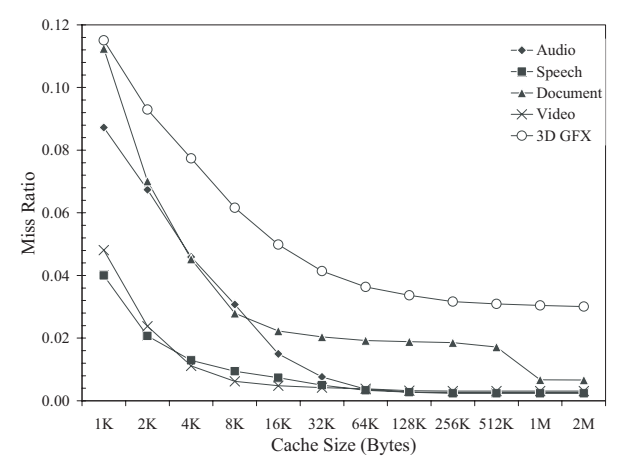

(c) Data Cache

Figure 5: Multimedia Domains - Line size is 32 bytes. The domains consist of $3 D$ graphics (Doom, Mesa, POVray), document (Ghostscript, DjVu, JPEG), audio (ADPCM, LAME, mpg123, Timidity), speech (Rsynth, GSM, Rasta) and video (MPEG-2).

erate them automatically; this will also effect their memory behavior.

\subsection{Line Size}

The block or line size of a cache memory is another cache design parameter that strongly affects cache performance [Smit87]. Generally, increasing the line size decreases the miss ratio, since each fetch from memory retrieves more data, thus fewer accesses outside the cache are required. When the line size is made too large, memory pollution can adversely affect cache performance, causing material to be loaded that is either never referenced or evicting information that would have been referenced before being replaced. Large lines also decrease the likelihood of "line crossers" - multibyte memory accesses across the boundary between two cache lines, such as occur with many CISC architectures. This type of access incurs a performance penalty since it usually requires two cache accesses; string operations can induce multiple cache data misses Additionally, small line sizes require a greater number of bits be dedicated to tag space than for larger lines, although a sector or sub-block cache is one way to avoid this problem. (See [Roth99a] for an investigation into sub-sector cache design issues.)

In addition to affecting the performance metric of miss ratio, large line sizes can have long transfer times and create excessively high levels of memory traffic [Smit87]. It is possible to model the time to fetch a cache line, $t_{\text {line }}$, assuming no prefetching and that all loads load a full cache line:

$$
t_{\text {line }}=t_{\text {latency }}+\frac{\left(\frac{L}{d}\right)}{r_{x f e r}}
$$

where,

$L$ - line size (bytes)

$d$ - data path width to memory (bytes)

$t_{\text {latency }}$ - delay for any memory transaction, consisting primarily of memory latency and address transmission time (seconds) $r_{x f e r}$ - bus transfer rate or bandwidth (bytes per second)

In order to select an optimal line size, it is necessary to minimize $t_{\text {line }} \cdot m(L)$, where $m(L)$ is the miss ratio as a function of line size. To investigate the effect of line size choice on miss ratio, instruction and data caches were simulated with line sizes ranging from 16 bytes to 256 bytes and total capacities ranging from $1 \mathrm{~KB}$ to $2 \mathrm{MB}$. As an example, we used the parameters measured for the memory hierarchy on a $500 \mathrm{MHz}$ AMD Athlon system, as listed in Table 3 . Because we are only considering one level caches in this work, we use the measured L2 parameters for the memory miss latency and bandwidth. For every cache capacity there is an optimal line size that minimizes the average memory reference delay. In the case of the largest caches simulated (1M and $2 \mathrm{M}$ capacity), the largest line size of 256 bytes produced minimal average delay for instruction caches. Table 6 summarize the mean memory reference delay for the multimedia workload for SPEC92, and SPEC95, in addition to the Berkeley Multimedia Workload. The best values are highlighted in bold text. Some of the instruction cache results exhibit anomalies for extremely small miss ratios due to the limited precision of our results in these instances (only a few misses for many millions of instruction references).

Our results indicate that for the Berkeley Multimedia Workload (as well as SPEC95), instruction cache line sizes should be as large as possible, due to the extremely low miss ratios exhibited for even moderate capacities. Instructions are likely to be accessed sequentially, so the fetching of large line sizes pays off. Data caches, on the other hand, have clearly optimal line sizes, depending on the total cache capacity. In the selection of an optimal line size, it should be kept in mind that large line sizes can be problematic in multiprocessor systems where system bus bandwidth must be shared. Very long line sizes may also cause real-time problems, as when I/O operations cause buffer overruns due to an inability to get on the memory bus. With many desktop computer manufacturers already offering 2 and even 4 -way multiprocessor support, 
this may have a limiting effect on the usefulness of long cache lines.

\subsection{Associativity}

Determining optimal associativity is important because changing associativity has a significant impact on cache performance (latency) and cost. Increasing set associativity may require additional multiplexing in the data path as well as increasing the complexity of timing and control [Przy88]. [Hill89] developed a rule of thumb for how associativity affects miss ratio: reducing associativity from eight-way to fourway, from four-way to two-way, and from two-way to direct mapped was found to cause relative miss ratio increases of approximately 5, 10, and 30 percent, respectively. In order to see how associativity affects miss ratios for our multimedia workload, miss ratio spreads were calculated for unified, data and instruction caches for our suite of multimedia applications. Miss ratio spread computes the benefit of increasing associativity, and is defined in [Hill89]:

$$
\text { Miss Ratio Spread }=\frac{m(A=n)-m(A=2 n)}{m(A=2 n)}
$$

Where $m(A=n)$ is the miss ratio for $n$-way set associativity, $A$. As in [Hill89], a block size of 32 bytes was chosen, with all simulated caches utilizing the LRU replacement algorithm. The miss ratio spreads of the Berkeley Multimedia Workload as well as SPEC92 and SPEC95 are shown in Figure 6. Please note that in order to preserve visual detail across the wide range of workload behaviors observed, the subfigures use different vertical scales. Unlike the original [Hill89] study, our curves are not smoothed or averaged.

From the miss ratio spread results in Figure 6, we can see that instruction caches for multimedia applications (and generally for SPEC92 and SPEC95) benefit from 2- or 4-way associativity for moderate size caches $(16 \mathrm{~KB}$ to $256 \mathrm{~KB})$. (Note that the scale is different for each portion of the figure.) For the multimedia workload, most of the benefit from associativity seems to be obtained with two-way set associativity; additional associativity doesn't seem to improve performance significantly, except for small cache sizes. Note that increasing associativity can also be a useful way to increase overall cache capacity when limited by virtual memory constraints (a limited number of page offset bits to index the cache). This was the approach taken both by the designers of Motorola's G4 processor (which includes 8-way associative L1 caches) as well as the IBM 3033 which has a 16-way associative $64 \mathrm{k}$ cache.

\section{Future Directions}

The final determination we would like to make is what cache designers should plan for in order to support future multimedia applications. We look at this in terms of the potential for data set expansion within each multimedia application domain. We expect that audio and speech application data sets will not change significantly in size, as current data sets are already at the limit of human audio fidelity. Document processing should also not change as current documents are sufficient for either printing or previewing at laser printer resolutions.

Video resolutions are not yet close to the limits of the human eye. This can be seen in the upcoming high resolution digital formats currently in the pipeline for consumer level products: DVD (720x480), HDTV $720 \mathrm{P}(1280 \times 720)$, and HDTV 1080I (1920x1080). In order to determine if the working set size of video applications is increasing, and therefore larger cache capacities are necessary to support these new resolutions, we compared the effect of cache capacity on miss ratios for them in Figures 7 and 8 utilizing the ratio of miss ratios for increasing resolution. Our results were obtained by running the same MPEG-2 decoding and encoding applications with three 30 frame data sets at DVD, HDTV 720P and HDTV 1080I resolutions. As an example of how to interpret the figures, DVD $\Rightarrow 720$ P refers to the ratio of miss ratios of $720 \mathrm{P} / \mathrm{DVD}$ resolutions. This metric shows the relative change in miss ratio for the higher resolution compared to the preceding lower resolution.

From Figure 7, we can see that instruction miss ratios are hardly affected at all by changes in resolution and although there are some minor fluctuations, the ratios are generally quite close to 1.0. Data miss ratios (Figure 8) show a stronger influence for small caches (capacities less than $32 \mathrm{~K}$ ), but level off for larger caches. This shows that the type of data locality being exploited by data caches for digital video is at the MPEG video block or macroblock level (which are the same size in all formats) rather than the frame level since caches are equally effective on all resolutions above a certain minimum working set size.

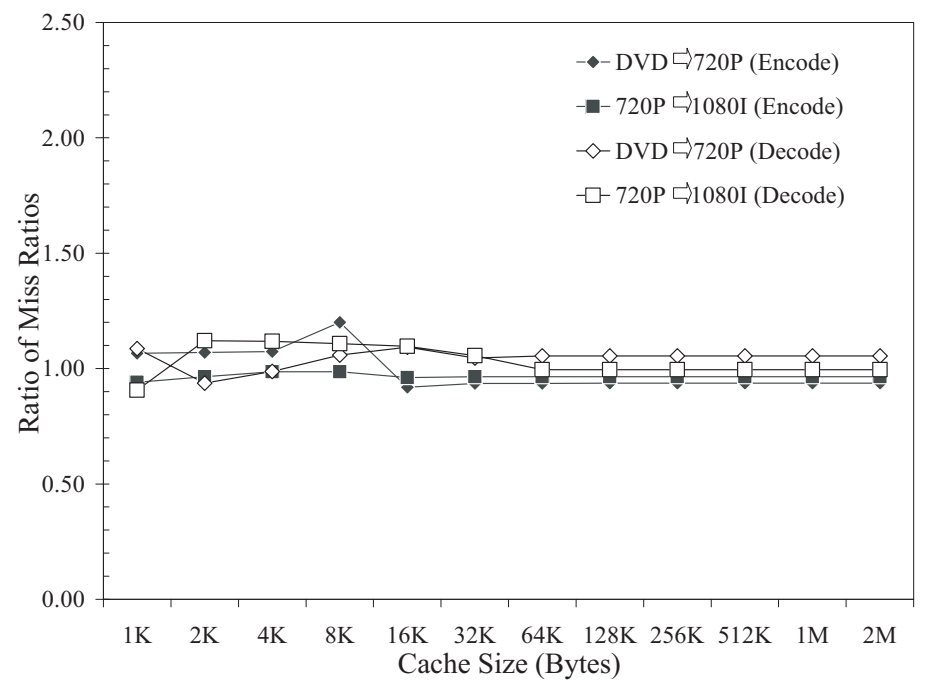

Figure 7: Digital Video Trends: Instruction Cache ratio of miss ratios for increasing resolution

Previous research ([Haku97], [Cox98], [Vart98]) has found that even a small texture cache located on a $3 \mathrm{D}$ accelerator board reduces the required bandwidth to main memory significantly. Past architectural trends suggest that all 3D rendering functionality will eventually be folded into the main 

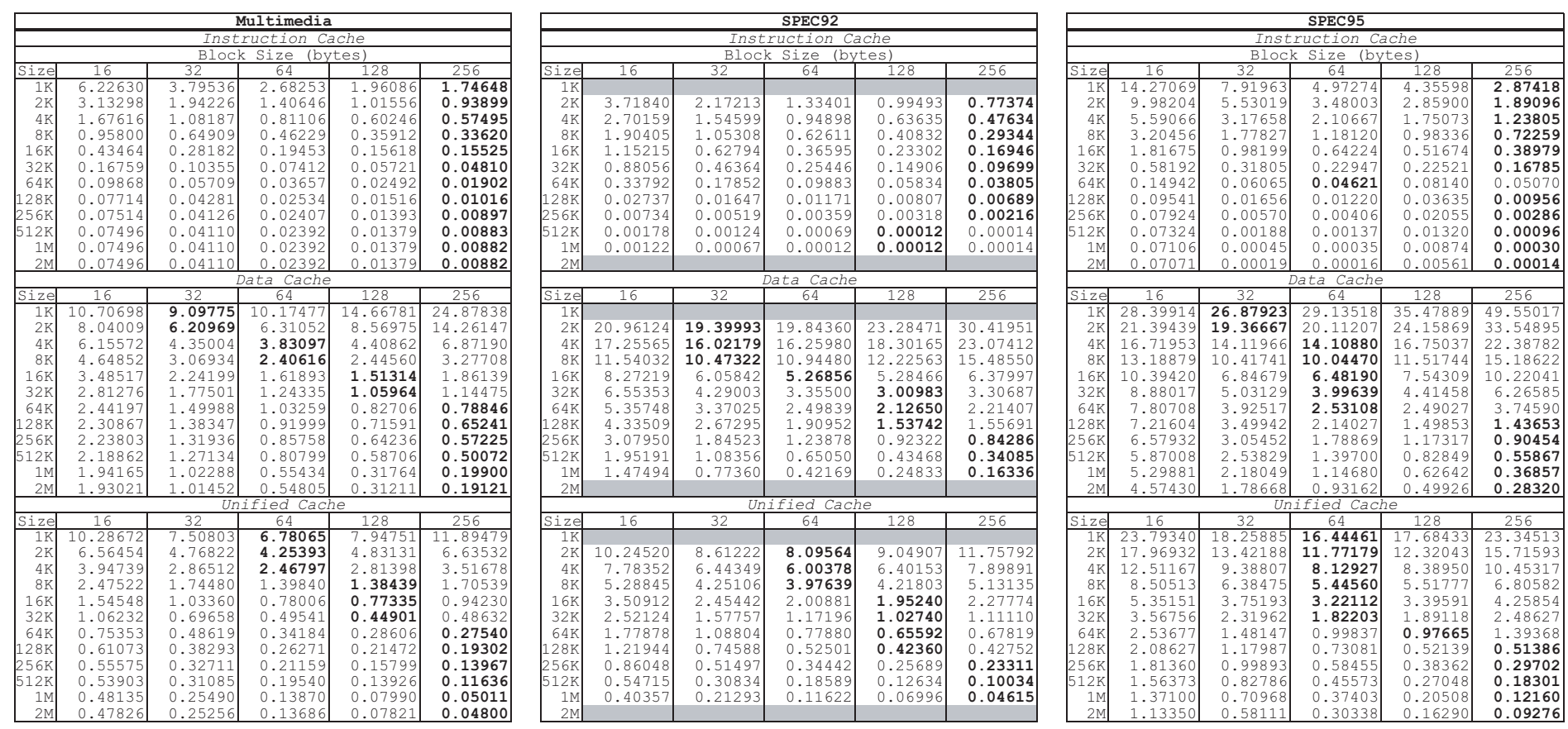

Table 6: Average Delay per Memory Reference (ns)

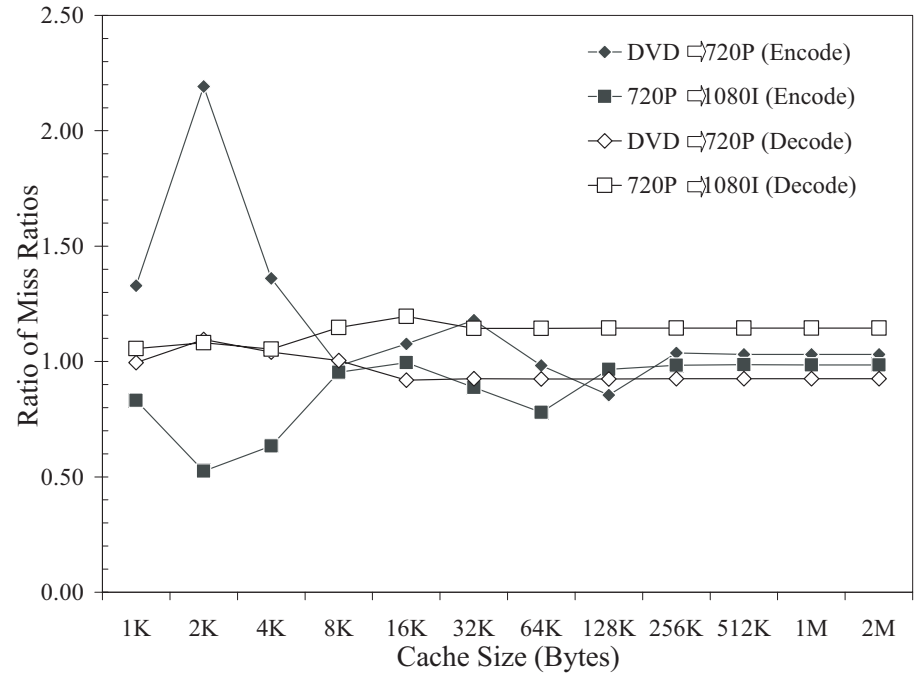

Figure 8: Digital Video Trends: Data Cache - ratio of miss ratios for increasing resolution

processor, at such time as there is adequate silicon to devote to it. We found that $3 \mathrm{D}$ applications exhibited the poorest locality of all the multimedia application types examined. Moving 3D functionality entirely into the CPU (and therefore sharing the cache with other applications) may require the reorganization of program structures to render vertices in an order amenable to LRU caching ([Haku97] examines several approaches for doing this) or larger caches to hold the substantial working sets of such applications. Texture size is dependent more upon the quality of rendered output rather than on display resolution, and is therefore subject to great pressure for growth [Intel].

\section{Summary}

In this paper we have provided a thorough analysis of three important cache parameters in order to support multimedia applications: cache capacity, line size and set associativity. Using execution driven simulation, a large design space was simulated incorporating multiprogramming effects.

\subsection{Capacity}

A moderate instruction cache capacity of $16 \mathrm{~KB}$ or $32 \mathrm{~KB}$ was found to be sufficient for all of the applications in our multimedia workload. Despite the widespread misconception that multimedia applications exhibit poor data cache performance, the Berkeley Multimedia Workload was found to exhibit quite low miss ratios. Optimal data cache size depends on the type of multimedia applications that are of interest. For the most common audio, speech and video multimedia applications, a data cache of $32 \mathrm{~KB}$ in capacity is large enough to exhibit extremely low $(<1 \%)$ miss ratios. Document and 3D processing exhibit less locality, and in fact even the largest cache sizes simulated (2 MB) still suffered significant misses for $3 \mathrm{D}$ graphics. As mentioned, this is due in large part to the fact that 3D graphics primitives (vertices) are processed in object order rather than memory order, leading to poor memory referencing behavior.

\subsection{Line Size}

We found benefit in instruction cache block sizes as large as the largest in our study ( 256 bytes) for the memory technol- 


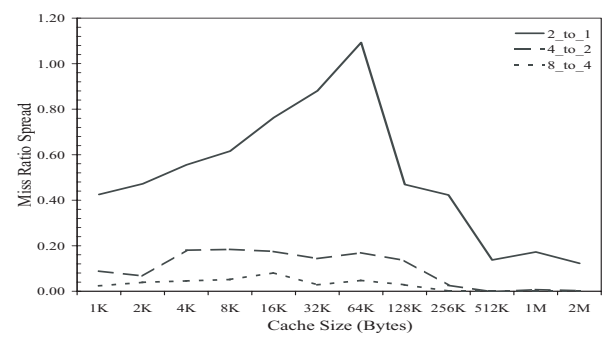

(a) Multimedia - Unified Cache

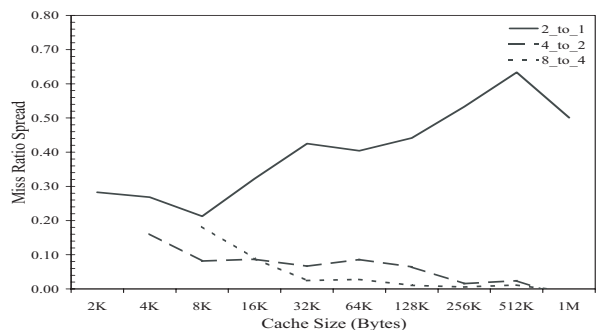

(d) SPEC92 - Unified Cache

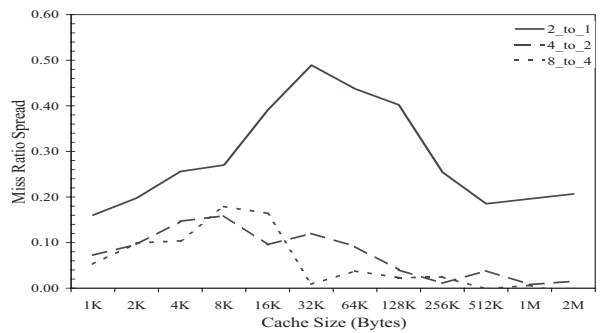

(g) SPEC95 - Unified Cache

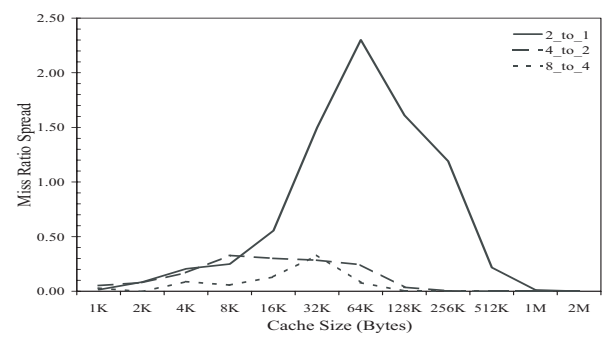

(b) Multimedia - Instruction Cache

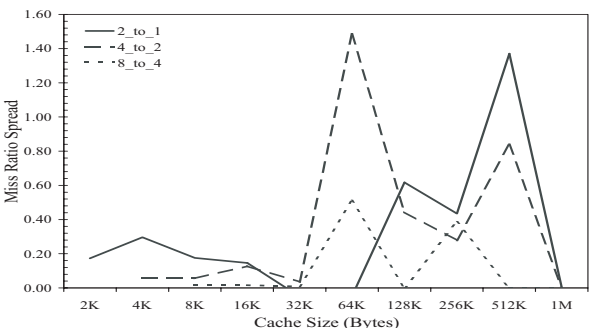

(e) SPEC92 - Instruction Cache

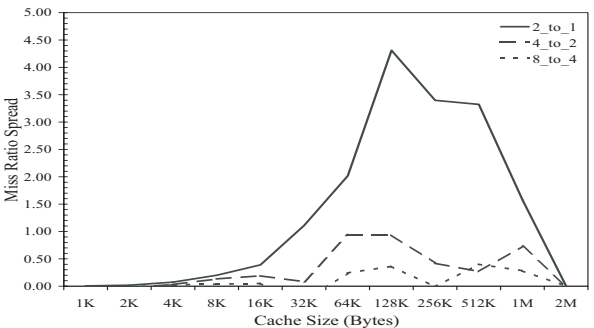

(h) SPEC95 - Instruction Cache

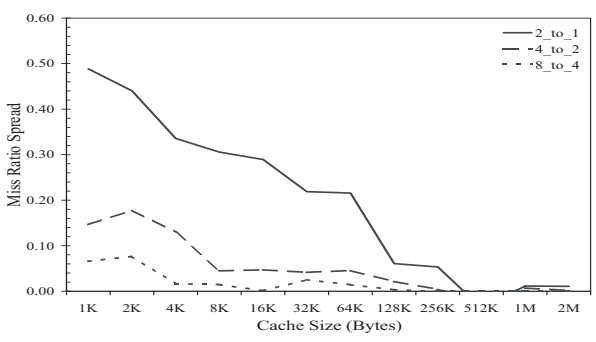

(c) Multimedia - Data Cache

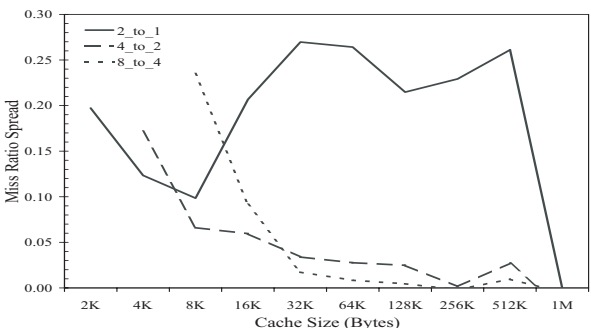

(f) SPEC92 - Data Cache

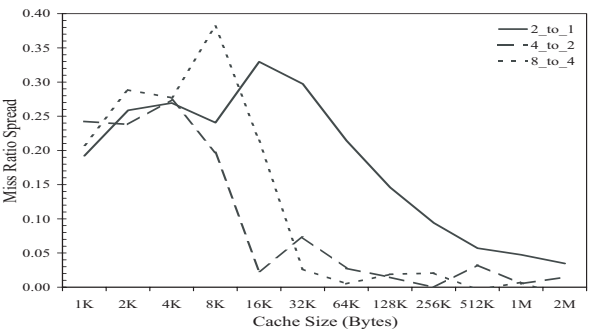

(i) SPEC95 - Data Cache

Figure 6: Berkeley Multimedia, SPEC92 and SPEC95 Miss Ratio Spreads - Each line, labeled $N$ to $M$, indicates the fraction increase in miss ratio when reducing associativity from $N$-way to $M$-way. To preserve detail across the wide range of workload behaviours observed, different vertical scales are used in each graph.

ogy examined at any cache capacity. Data cache block size selection is more dependent on the capacity of the cache. It is important to note that our block size choices considered only average memory access time, and did not consider issues such as total memory traffic or bus busy periods, which are important considerations for multiprocessor machines.

\subsection{Associativity}

Based on the results of the miss ratio spread analysis, instruction caches can optimally benefit from 2- or 4-way associativity for most moderate cache sizes (16 KB to $256 \mathrm{~KB})$. Data cache benefits from varying degrees of associativity are more difficult to generalize and appear to be highly dependent on the specific workload, but in general, 2-4 way associativity is also a good choice.

\section{References}

[Aust] Todd M. Austin, Doug Burger, Manoj Franklin, Scott Breach, Kevin Skadron, "The SimpleScalar Architectural Research Tool Set," http://www.cs.wisc.edu/ ${ }^{\sim}$ sscalar/simplescalar.html, retrieved April 24, 2000

[Bhas97] Vasudev Bhaskaran, Konstantinos Konstantinides, and Balas Natarajan, "Multimedia architectures: from desktop systems to portable appliances," Proc. Multimedia Hardware Architectures, San Jose, California, February 2-14, 1997, SPIE Vol. 3021, pp. $14-25$

[Cont97] Thomas M. Conte, Pradeep K. Dubey, Matthew D. Jennings, Ruby B. Lee, Alex Peleg, Salliah Rathnam, Mike Schlansker, Peter Song, Andrew Wolfe, "Challenges to Combining GeneralPurpose and Multimedia Processors," IEEE Computer, Vol. 30, No. 12, December 1997, pp. 33-37

[Cox98] Michael Cox, Narendra Bhandari, Michael Shantz, "Multi-Level Texture Caching for 3D Graphics Hardware," Proc. 25th Int'l Symp. on Computer Architecture, Barcelona, Spain, June 27July 1, 1998, pp. 86-97

[Cucc99] Rita Cucchiara, Massimo Piccardi, Andrea Prati, "Exploiting Cache in Multimedia," Proc. of IEEE Multimedia Systems '99 Vol. 1, Florennce, Italy, July 7-11 1999, pp. 345-350 
$[\mathrm{DEC}]$

Digital Equipment

ence Manual, Corporation, Referswdev/files/DECOSF1/Docs/Other/ATOM/ref.ps retrieved April 24, 2000

[Dief97] Keith Diefendorff, Pradeep K. Dubey, "How Multimedia Workloads Will Change Processor Design," IEEE Computer, Vol. 30, No. 9, September 1997 , pp. 43-45

[Edler] Jan Edler, Mark D. Hill, "Dinero IV Trace-Driven Uniprocessor Cache Simulator," http://www.neci.nj.nec.com/homepages/edler/d4/, retrieved Apil 24, 2000

[Gee93] Jeffrey D. Gee, Mark D. Hill, Dionisios N. Pnevmatikatos, Alan Jay Smith, "Cache Performance of the SPEC92 Benchmark Suite," IEEE Micro, Vol. 13, No. 4, August 1993, pp. 17-27

[Haku97] Ziyad S. Hakura, Annop Gupta, "The Design and Analysis of a Cache Architecture for Texture Mapping," Proc. 24th Int'l Symp. on Computer Architecture, Denver, Colorado, June 2-4, 1997, pp. $108-120$

[Hill89] Mark D. Hill, Alan Jay Smith, "Evaluating Associativity in CPU Caches," IEEE Trans. on Computers, Vol. 38, No. 12, December 1989 , pp. $1612-1630$

[Intel] Intel Corporation, "Accelerated Graphics Port Interface Specification v2.0," May 4, 1998 , http://developer.intel.com/technology/agp/, retrieved April 24, 2000

[Kess91] Richard Eugene Kessler, "Analysis of Multi-Megabyte Secondary CPU Cache Memories," University of Wisconsin-Madison Computer Sciences Technical Report \#1032, July 1991

[Kuro98] Ichiro Kuroda, Takao Nishitani, "Multimedia Processors," Proc. IEEE, Vol. 86, No. 6, June 1998, pp. 1203-1221

[Lee96] Ruby B. Lee, Michael D. Smith, "Media Processing: A New Design Target," IEEE Micro, Vol. 16, No. 4, August 1996, pp. 6-9

[Przy88] Steven Przybylski, Mark Horowitz, John Hennessy, "Performance Tradeoffs in Cache Design," Proc. 15th Annual Int'l Symp. on Computer Architecture, Honolulu Hawaii, May 30-June 2, 1988 pp. $290-298$

[Rixn98] Scott Rixner, William J. Dally, Ujval J. Kapasi, Brucek Khailany, Abelardo Lopez-Lagunas, Peter R. Mattson, John D. Owens, "A Bandwidth-Efficient Architecture for Media Processing," Proc. 31st Int'l Symp. on Microarchitecture, Dallas, Texas, November 30-December 2, 1998, pp. 3-13

[Roth99a] Jeffrey B. Rothman, Alan Jay Smith, "The Pool of Subsectors Cache Design," Proc. Int'l Conference on Supercomputing, Rhodes, Greece, June 20-25 1999, pp. 31-42

[Roth99b] Jeffrey B. Rothman, Alan Jay Smith, "Multiprocessor Memory Reference Generation Using Cerberus," Proc. 7th Int'l Symp. on Modeling, Analysis and Simulation of Computer and Telecommunication Systems (MASCOTS'99), College Park, Maryland, October 24-28, 1999, pp. 278-287

[Sling00a] Nathan T. Slingerland, Alan Jay Smith, "Design and Characterization of the Berkeley Multimedia Workload," University of California at Berkeley Technical Report CSD-00-1122, December 2000

[Smit82] Alan Jay Smith, "Cache Memories," ACM Computing Surveys, Vol. 14, No. 3, September 1982, pp. 473-530

[Smit87] Alan Jay Smith, "Line (Block) Size Choice for CPU Cache Memories," IEEE Trans. on Computers, Vol. C-36, No. 9, September 1987, pp. $1063-1075$

[Smit94] Alan Jay Smith, "Trace Driven Simulation in Research on Computer Architecture and Operating Systems," Proc. Conference on New Directions in Simulation for Manufacturing and Communications, Tokyo, Japan, August 1994, pp. 43-49

[Sode97] Peter Soderquist, Miriam Leeser, "Optimizing the Data Cache Performance of a Software MPEG-2 Video Decoder," Proc. ACM Multimedia '97, Seattle, Washington, November 9-13, 1997, pp. 291-301

[Stru98] Pieter Struik, Pieter van der Wolf, Andy D. Pimentel, "A Combined Hardware/Software Solution for Stream Prefetching in Multimedia Applications." Proc. 10th Annual Symp. on Electronic Imaging, San Jose, California, January 1998, pp. 120-130

[Sugu93] Rabin A. Sugumar, Santosh G. Abraham, "Efficient Simulation of Caches under Optimal Replacement with Applications to Miss Characterization," Proc. 1993 ACM Sigmetrics Conference on Measurements and Modeling of Computer Systems, May 1993, pp. 24-35

[Tse98] J. Tse, A. J. Smith, "CPU cache prefetching: Timing evaluation of hardware implementations," IEEE Trans. on Computers, Vol. 47, No.5, May 1998. pp. 509-26

[Uhli97] Richard Uhlig, Trevor Mudge, "Trace-Driven Memory Simulation: A Survey", ACM Computing Surveys, Vol. 29, No. 2, June 1997, pp. $128-170$
[Vart98] Alexis Vartanian, Jean-Luc Bechennec, Natalie Drach-Temam, "Evaluation of High Performance Multicache Parallel Texture Mapping," Proc. 1998 Int'l Conference on Supercomputing, Melbourne, Australia, July 13-17, 1998, pp. 289-296

[WARTS] Glenn Ammons, Tom Ball, Mark Hill, Babak Falsafi, Steve HussLederman, James Larus, Alvin Lebeck, Mike Litzkow, Shubhendu Mukherjee, Steven Reinhardt, Madhusudhan Talluri, and David Wood, "WARTS: Wisconsin Architectural Research Tool Set," http://www.cs.wisc.edu/ larus/warts.html, retrieved April 24, 2000

[Zuck96] Daniel F. Zucker, Michael J. Flynn, and Ruby B. Lee, "A Comparison of Hardware Prefetching Techniques for Multimedia Benchmarks," Proc. 3rd IEEE Int'l Conference on Multimedia Computing and Systems, Hiroshima, Japan, June 17-23, 1996 pp. 236-244 


\section{Appendix}

\subsection{Context Switch Intervals}

[Agar88] found that actual multiprogramming traces showed a wide variation in time slice duration and execution order of processes. Although the level of multiprogramming on a desktop workstation is typically low, multimedia applications are often multi-threaded, such that in the example of DVD playback, one thread handles bit stream parsing, another video decoding, and still another audio decoding. Multimedia applications are time dependent; their output or input of processed multimedia data must be synchronized with production or consumption of that data. For this reason, the length of the context interval (utilized portion of the quantum) depends on $\mathrm{I} / \mathrm{O}$ device interaction, number of component threads, and amount of computation time necessary to operate on a given unit of data time.

\subsubsection{Windows 2000 Multimedia}

In order to estimate typical context switch intervals for real multimedia applications, a set of commercial multimedia applications corresponding to those in the Berkeley Multimedia Workload were measured. All runs were with a single active process running. The context switch intervals of the actual applications from the Berkeley Multimedia Workload were not measured because they are primarily file based applications, typically converting between compressed and uncompressed format without presenting the resulting data to the user. So, although the algorithms they employ (and therefore their memory access patterns) should for the most part be realistically similar to their commercial counterparts, their scheduling behavior is vastly different. For example, in the case of on screen DVD movie play back, there are typically several concurrent threads of execution, each dealing with a particular aspect of MPEG-2 decoding (e.g. audio, video, bitstream parsing/demuxing). Acceptable playback requires that for each unit of data set time each decoding component must be computed:

1. fast enough to leave time for computing the other components in that unit of time (otherwise video frames may need to be dropped)

2. with sufficient precision to not introduce noticable artifacts

3. within a given time frame to prevent latency effects from disrupting the perceived synchronization between audio and video.

These requirement affect scheduling, and are not taken into account in an application which operates in a batch or offline mode.

Context switch intervals were measured on a $500 \mathrm{MHz}$ AMD Athlon with $256 \mathrm{MB}$ of PC100 DRAM and an MSI MS-6167 motherboard running Windows 2000 Professional v5.00.2195. Both a sound card (Sound Blaster Live Value) and 3D accelerator card (AGP Nvidia Riva TNT) were installed. Table 2 lists these intervals as measured by the Windows 2000 performance counters, which return results in terms of time (CPU cycles).

\begin{tabular}{|c|c|}
\hline CINT95 & Context Interval \\
\hline 099.go & $21,134,208$ \\
\hline $124 . \mathrm{m} 88 \mathrm{ksim}$ & $5,122,455$ \\
\hline 126.gcc & $3,845,678$ \\
\hline 129.compress & $22,719,364$ \\
\hline 130.li & $21,754,551$ \\
\hline 132.ijpeg & $16,093,926$ \\
\hline 134.perl & $16,308,625$ \\
\hline 147.vortex & $13,193,123$ \\
\hline CFP95 & Context Interval \\
\hline 101.tomcatv & $10,185,364$ \\
\hline 102.swim & $13,753,700$ \\
\hline 103.su2cor & $9,595,431$ \\
\hline 104.hydro2d & $18,624,108$ \\
\hline 107.mgrid & $17,791,106$ \\
\hline 110.applu & $4,644,660$ \\
\hline 125.turb3d & $22,366,853$ \\
\hline 141.apsi & $11,743,787$ \\
\hline 145.fpppp & $19,004,011$ \\
\hline 146. wave5 & $19,015,575$ \\
\hline Arithmetic Mean & $2,243,433$ \\
\hline Geometric Mean & $2,238,412$ \\
\hline
\end{tabular}

Table 1: Average SPEC95 Context Switch Intervals measurements are given in $500 \mathrm{MHz}$ clock cycles

In our cache simulations, we simulate normal task switching by flushing the cache every $Q_{\text {context }}$ instructions. Because our cache simulation is instruction, rather than cycle based, we require cache purge intervals measured in terms of instructions executed between cache flushes. In order to convert our context switch interval data from cycles to instructions we need to know the corresponding cycles per instruction (CPI) ratio. However, we can not simply treat x86 CISC instructions as being equivalent to RISC Alpha instructions, due to the inherently different amounts of work done by each class of instructions. Interestingly, although the $\mathrm{x} 86$ instruction set architecture that the AMD Athlon executes is CISC in nature, the full CISC instructions are internally decoded by the Athlon processor into RISC-like instructions referred to as micro-ops or simply ops [AMD]. Thus, in order to approximate the equivalent number of Alpha RISC-like instructions in each context switch interval, we divide the number of cycles by the number of cycles per micro-op $(\mathrm{CP} \mu \mathrm{Op})$.

In order to measure a representative value of cycles per micro-op, the same AMD Athlon system as was used to run the Windows workload was measured running the Berkeley Multimedia Workload on Mandrake Linux v7.0. This system was then modified to use kernel version 2.2.15 with Mikael Petterson's perfect counters patch v1.2 [Pett]. The AMD Athlon (as well as many other processors) implement hardware performance counters which can keep track of statistics 


\begin{tabular}{|c|c|c|}
\hline Application Name & Data Set & Context Interval \\
\hline 3D Flowerbox OpenGL Screen Saver (Windows 2000) & $1280 \times 1024 \times 32 \mathrm{bpp},(1: 00)$ & 23,653 \\
\hline RealPlayer v7.0 RealAudio Player & KAMU $64 \mathrm{Kbps}$, stereo, G2 stream, (5:00) & 40,396 \\
\hline Real Jukebox v1.0.0.488 MP3 Player & Santana - Smooth, $160 \mathrm{Kbps}$, stereo (4:54) & 58,399 \\
\hline MediaPlayer GSM 06.10 (Windows 2000) & Speech by Al Gore, $8 \mathrm{kHz}$ Mono, 16-bits $(0: 24)$ & 297,641 \\
\hline K-Jofol 2000 MP3 Player v1.0 & Santana - Smooth, $160 \mathrm{Kbps}$, stereo (4:54) & 360,336 \\
\hline 3D Pipes OpenGL Screen Saver (Windows 2000) & $1280 \times 1024 \times 32 b p p,(1: 00)$ & 567,080 \\
\hline Narrator Text to Speech (Windows 2000) & U.S. Declaration of Independence & 594,438 \\
\hline MediaPlayer IMA ADPCM (Windows 2000) & Santana - Smooth, $160 \mathrm{Kbps}$, stereo $(4: 54)$ & 708,037 \\
\hline WinDVD v2.0 DVD Player & (5:00) clip from Amadeus & 921,510 \\
\hline PowerDVD v2.55 DVD Player & (5:00) clip from Amadeus & $1,189,234$ \\
\hline Ghostscript/Ghostview PostScript Previewer & Rosenblum and Ousterhaut's LFS paper (15 pages) & $1,227,194$ \\
\hline Dragon Naturally Speaking Preferred Speech Recognition & U.S. Declaration of Independence & $2,560,537$ \\
\hline Audio Catalyst v2.1 MP3 Encoder & Santana - Smooth, $44 \mathrm{kHz}$, stereo (4:54) & $3,358,692$ \\
\hline Audio Compositor MIDI Renderer & X-files theme song, Personal Copy v4.2 Sound Fonts & $3,675,086$ \\
\hline Irfanview v3.15 Image Viewer & Kodak's Iowa Corn jpeg image (2048x3072x24bpp) & $3,821,284$ \\
\hline Quake III Arena (Demo) & Internal demo $\# 1$, demo $\# 2(640 \times 480)$ & $4,284,671$ \\
\hline DjVushop Document Compression & Scanned cover of March 2000 IEEE Computer journal & $4,754,521$ \\
\hline Avi2Mpg2 MPEG-2 Encoder & 160 frames, $720 \times 480$ from Amadeus & $5,339,432$ \\
\hline POVray v3.1g Raytracer & Torus (internal demo scene), 800x600 Anti-Aliased & $5,928,433$ \\
\hline 3D Maze OpenGL Screen Saver (Windows 2000) & $1280 \times 1024 \times 32 b p p,(1: 00)$ & $5,930,096$ \\
\hline \multirow{2}{*}{\multicolumn{2}{|c|}{$\begin{array}{rr}\text { Arithmetic Mean } \\
\text { Geometric Mean }\end{array}$}} & $2,247,389$ \\
\hline & & $1,015,426$ \\
\hline
\end{tabular}

Table 2: Average Multimedia Context Switch Intervals - Measurements are given in $500 \mathrm{MHz}$ clock cycles. Applications annotated with "(Windows 2000)" are packaged with the Windows 2000 operating system.

such as instructions executed, micro-ops executed, as well as many others [AMD]. (These hardware performance counters are not readily accessible under Windows.) The results of these measurements are shown in Table 3, which lists data both in terms of instructions as well as micro-ops.

In order to determine $Q_{\text {context }}$ for the RISC Alpha architecture, we divide the context switch intervals in Table 2 by a $\mathrm{CPI}$ equal to the average $1.06 \mathrm{CP} \mu \mathrm{Op}$ measurement from Table 3. As stated earlier, we use this parameter rather than x86 CPI because micro-ops are much more similar to RISC Alpha instructions than CISC x86 instructions. Note also that in a real system the interval between task switches is variable, not fixed; since we don't have the distribution of inter-interrupt times, we chose to use a constant interval. Alternately, we could have chosen some other distribution, such as exponential, normal or uniform.

\subsubsection{Compaq Unix SPEC95}

Table 1 lists context switch intervals for SPEC95 measured for Compaq Tru64 Unix v5.0 running on a DEC DS20 workstation (dual 21264 processors, each running at $500 \mathrm{MHz}$ ), with 2 GB of RAM, again running in a system with a single active task. For comparison, we review below the multiprogramming parameters of a variety of other workloads and systems previously studied in the literature. [Agar88] found that task switch times varied between 10,000 and 50,000 instruction references for both DEC Ultrix and VMS operating systems on a VAX 8200 processor $(5 \mathrm{MHz})$. [Borg90] lists parameters for Titan, an experimental RISC workstation with a clock speed of $22.2 \mathrm{MHz}$, for which $Q=25,000$ clock cycles, with CPI typically being greater than one. [Clark83] measured context switch intervals between 11,000 and 23,000 instruction references on the DEC VAX $11 / 780(5 \mathrm{MHz})$. Later measurements by the same author in [Clark88] on a VAX 8800 processor $(22.2 \mathrm{MHz})$ found an average of 19,353 instructions between context switches. The traces used in [Smit87] were from a variety of architectures (Zilog Z8000, DEC VAX 11/780, CDC 6400, IBM 370, and Motorola 68000), and were simulated with a task switch interval of $15,000-20,000$ memory references. [Koba86] examines the task switching behavior of IBM's MVS operating system (OS/VS2 MVS) on an Amdahl $470 \mathrm{~V} / 8$ system. Measurements obtained by a hardware monitor for a production time sharing option (TSO) workload had task intervals of approximately 7,600 instructions and 3,600 instructions for supervisor (system) state and problem (user) state respectively. A batch workload exhibited 8,700 and 14,000 for supervisor and problem state. Note that all of these other workloads were heavily multiprogrammed (and timeshared), and thus are not fully comparable. Of course, much computation is currently done on single user PCs and workstations, so a uniprogrammed system is probably the most representative of current technology.

\subsection{Simulation Methodologies}

Even though our study utilizes execution driven simulation, tracing full applications is still costly in terms of simulation 


\begin{tabular}{|c|c|c|c|c|c|}
\hline Name & User Time (cycles) & Instructions & Micro-Ops & Cycles/Instruction & Cycles/Micro-Op \\
\hline ADPCM Encode & $76,904,958$ & $61,876,844$ & $62,158,059$ & 1.24 & 1.24 \\
\hline ADPCM Decode & $64,344,819$ & $53,313,197$ & $53,594,784$ & 1.21 & 1.20 \\
\hline DJVU Encode & $445,708,199$ & $287,174,703$ & $322,253,494$ & 1.55 & 1.38 \\
\hline DJVU Decode & $356,305,000$ & $244,918,278$ & $266,984,846$ & 1.45 & 1.33 \\
\hline Doom & $1,472,798,700$ & $793,162,073$ & $1,181,637,518$ & 1.86 & 1.25 \\
\hline Ghostscript & $670,630,530$ & $748,440,530$ & $827,890,835$ & 0.90 & 1.81 \\
\hline GSM Encode & $188,190,001$ & $225,158,845$ & $248,092,796$ & 0.84 & 0.76 \\
\hline GSM Decode & $60,417,117$ & $83,563,455$ & $87,869,424$ & 0.72 & 0.69 \\
\hline JPEG Encode & $153,366,654$ & $155,941,646$ & $172,197,756$ & 0.98 & 0.89 \\
\hline JPEG Decode & $78,532,637$ & $69,994,886$ & $71,219,757$ & 1.12 & 1.10 \\
\hline LAME & $10,695,164,749$ & $8,758,744,400$ & $9,792,965,536$ & 1.22 & 1.09 \\
\hline Mesa Gears & $607,508,141$ & $238,630,536$ & $246,478,641$ & 2.55 & 2.46 \\
\hline Mesa Morph3D & $430,137,133$ & $200,480,866$ & $247,112,920$ & 2.15 & 1.74 \\
\hline Mesa Reflect & $1,940,806,991$ & $1,692,447,264$ & $2,090,552,059$ & 1.15 & 0.93 \\
\hline MPEG-2 Encode DVD & $14,037,454,521$ & $14,005,949,265$ & $16,702,192,536$ & 1.00 & 0.84 \\
\hline MPEG-2 Encode $720 \mathrm{P}$ & $37,759,708,362$ & $37,445,342,666$ & $44,698,434,032$ & 1.01 & 0.84 \\
\hline MPEG-2 Encode 1080I & $85,720,663,990$ & $87,173,068,231$ & $103,429,470,721$ & 0.98 & 0.83 \\
\hline MPEG-2 Decode DVD & $778,728,056$ & $930,444,099$ & $974,763,847$ & 0.84 & 0.80 \\
\hline MPEG-2 Decode $720 \mathrm{P}$ & $2,462,187,286$ & $2,937,962,420$ & $3,110,807,374$ & 0.84 & 0.79 \\
\hline MPEG-2 Decode $1080 \mathrm{I}$ & $5,043,716,605$ & $5,759,111,626$ & $6,014,852,989$ & 0.88 & 0.84 \\
\hline $\operatorname{mpg} 123$ & $446,758,849$ & $409,577,365$ & $486,650,398$ & 1.09 & 0.92 \\
\hline POVray3 & $72,427,881,123$ & $5,137,007,093$ & $6,809,522,382$ & 1.45 & 1.09 \\
\hline Rasta & $27,180,004$ & $21,569,326$ & $26,527,625$ & 1.26 & 1.02 \\
\hline Rsynth & $526,040,697$ & $421,248,076$ & $534,184,372$ & 1.25 & 0.98 \\
\hline Timidity & $1,132,954,552$ & $1,363,791,599$ & $1,522,840,735$ & 0.83 & 0.74 \\
\hline \multicolumn{4}{|c|}{$\begin{array}{ll} & \\
& \text { Average } \\
\end{array}$} & 1.21 & 1.06 \\
\hline
\end{tabular}

Table 3: Measured CPI and $\mathbf{C P} \mu \mathrm{Op}$ for Berkeley Multimedia Workload - $500 \mathrm{MHz}$ AMD Athlon system, $128 \mathrm{MB}$ RAM, running Mandrake Linux v7.0 with a modified kernel v2.2.15

time. Our cache simulations were run on a lab of twenty Alpha AXP 300 workstations at Berkeley, each with a single $300 \mathrm{MHz}$ Alpha 21064a processor and $128 \mathrm{MB}$ of RAM. Simulation time per memory reference is dependent on the type of cache being simulated, requiring $0.418 \mu \mathrm{s}$ (126 cycles), $1.770 \mu \mathrm{s}$ (531 cycles), and $1.262 \mu \mathrm{s}$ (379 cycles) on average per reference for instruction, data and unified cache simulations respectively. Instruction cache simulation is especially fast because references can be simulated at the basic block level of granularity rather than per instruction as is necessary for data references. Average simulation time per reference was between $0.114 \mu \mathrm{s}$ (35 cycles) and $2.112 \mu \mathrm{s}$ (634 cycles) (for comparison, [Gee93]'s simulation of SPEC92 on $40 \mathrm{MHz}$ DECstation 5000/240 machines required $200 \mu \mathrm{s}$ (8000 cycles) to $400 \mu$ s $(16,000$ cycles $)$ per reference simulated for simulations). Total simulation time for our work, not including false starts, machine down time and other simulation problems was 24.4 days of $\mathrm{CPU}$ time for each multimedia run (once with and once without multiprogramming), and 147.2 days of CPU time for SPEC95 simulations, for a grand total of 196 days of CPU time.

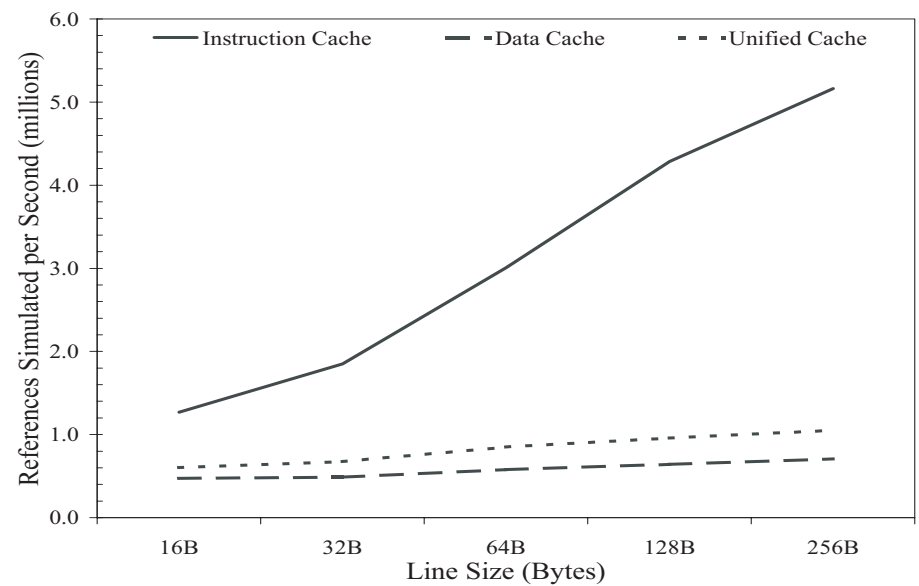

Figure 1: Average ATOM Cheetah Simulation Speeds

\subsection{Current Cache Configurations}

As can be seen from Table 4, currently available processors are very similar in their cache design choices. 


\begin{tabular}{|l|c|c|c|c|c|c|c|}
\hline & $\begin{array}{c}\text { AMD } \\
\text { Athlon }\end{array}$ & $\begin{array}{c}\text { DEC } \\
\mathbf{2 1 2 6 4 A}\end{array}$ & $\begin{array}{c}\text { HP } \\
\text { PA-8500 }\end{array}$ & $\begin{array}{c}\text { Intel } \\
\text { Pentium III }\end{array}$ & $\begin{array}{c}\text { MIPS } \\
\text { R12000 }\end{array}$ & $\begin{array}{c}\text { Motorola } \\
\text { 7400 (G4) }\end{array}$ & $\begin{array}{c}\text { Sun } \\
\text { UltraSPARC IIi }\end{array}$ \\
\hline \hline Transistors $\left(x 10^{6}\right)$ & 22 & 15.2 & 140 & 9.5 & 6.9 & 6.5 & 5.4 \\
\hline Process $(\mu \mathrm{m})$ & 0.25 & 0.25 & 0.25 & 0.18 & 0.25 & 0.20 & 0.25 \\
\hline Die Size $\left(\mathrm{mm}^{2}\right)$ & 184 & 205 & 468.6 & 104.6 & 204 & 83 & 126 \\
\hline Clock $(\mathrm{MHz})$ & 800 & 750 & 440 & 733 & 300 & 500 & 480 \\
\hline L1 \$I Size $(\mathrm{KB})$ & 64 & 64 & 512 & 16 & 32 & 32 & 16 \\
\hline L1 \$I Associativity & 2 & 2 & 4 & 4 & 2 & 8 & 2 \\
\hline L1 \$I Line Size (bytes) & 64 & 16 & $32 / 64$ & 32 & 32 & 32 & 32 \\
\hline L1 \$D Size (KB) & 64 & 64 & 1024 & 16 & 32 & 32 & 16 \\
\hline L1 \$D Associativity & 2 & 2 & 4 & 4 & 2 & 8 & 1 \\
\hline L1 \$D Line Size (bytes) & 64 & 64 & $32 / 64$ & 32 & 32 & 32 & 32 \\
\hline
\end{tabular}

Table 4: Current Microprocessor Cache Configurations [Burd][Noer]

\subsection{Measuring Cache Parameters}

In order to choose the optimal line size for a given cache size, it is first necessary to know the parameters of the memory system to be used. In order to illustrate this, we employed HBench-OS v1.0 [Brow97], an extended version of the lmbench micro-benchmarking suite detailed in [McVoy96] (this work was apparently developed independently from the TLB and cache measurement techniques presented in [Saav95]). Each micro-benchmark measures the average time per iteration required to read or write a subset of elements belonging to an array of known size. The number of cache misses (and therefore the latency) is a function of the size of the array and the stride between consecutive addresses referenced. This effect can be seen in Figure 2 which graphs latency for various stride sizes for the $500 \mathrm{MHz}$ AMD Athlon system utilized in our study. Each data set curve represents a stride size with the array size varying from 512 bytes up to 8 Mbytes, with a latency of 1 clock cycle taken to be "zero" - the time reported is only memory latency time, and does not include the instruction execution time. This is why all of the curves in Figure 2 are zero left of the L1 data cache capacity (64 Kbytes). Each horizontal plateau represents another level of the memory hierarchy (L1 at 64 Kbytes, L2 at 512 Kbytes for the AMD Athlon). Different strides are measured by the benchmark in order to determine the cache line size. The smallest stride that has a latency equal to that of main memory is the cache line size because the strides that are faster than memory must necessarily hit more than once per cache line.

\subsection{Comparison with [Smit87]}

In order to compare our line size analysis results with other workloads, we examined the metric of ratio of miss ratios [Smit87], which is the ratio of the miss ratio at a given cache size and line size to that for the same cache size but half the line size. The analysis of line size in [Smit87] employs a set of 27 traces from five different machine architectures. Table 5 compares the ratio of miss ratios metric for that workload against those of our multimedia workload. Graphs of the ratios of miss ratios for the Berkeley Multimedia Workload

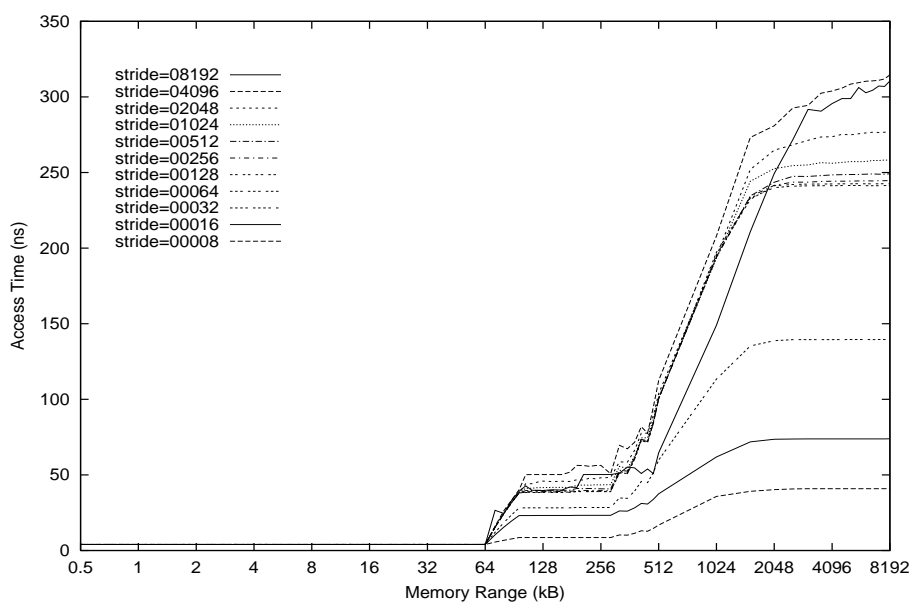

Figure 2: $500 \mathrm{MHz}$ Athlon System Memory Latency Profile - memory latency for various stride length memory accesses

are shown in Figures 3, 4, and 5. Cache behavior was quite similar between the two workloads except in the case of data caches. The multimedia workload benefitted more than the one studied in [Smit87] when going from 16B to 32B data cache line lengths, but the opposite held true for the other data cache line length comparisons (64B:32B and 128B:64B).

\subsection{Model}

It is useful to examine the miss ratio spreads for the multimedia, SPEC92 and SPEC95 workloads in light of the three C's model of cache miss behavior introduced in [Hill87] which categorizes misses into three classes: conflict misses, which are due to two different elements of the working set mapping to the same physical cache blocks (none for a fully associative cache), capacity misses which result from the cache capacity being to small to hold the working set (none for an infinitely large cache) and compulsory misses which are necessary when initially loading the cache (present for any cache configuration). It is only where conflict misses exist that associativity makes a difference, and there is a great variation among different applications in the contribution conflict misses make 


\begin{tabular}{|c|c|c|c|c|c|c|}
\hline \multicolumn{7}{|c|}{$32 \mathrm{~B}: 16 \mathrm{~B}$} \\
\hline & \multicolumn{2}{|c|}{ Unified } & \multicolumn{2}{|c|}{ Instruction } & \multicolumn{2}{|c|}{ Data } \\
\hline Size & Media & [Smit87] & Media & [Smit87] & Media & [Smit87] \\
\hline $1 \mathrm{~K}$ & 0.676 & 0.831 & 0.597 & 0.963 & 0.789 & 0.836 \\
\hline $2 \mathrm{~K}$ & 0.679 & 0.731 & 0.607 & 0.737 & 0.670 & 0.787 \\
\hline $4 \mathrm{~K}$ & 0.727 & 0.719 & 0.673 & 0.651 & 0.657 & 0.727 \\
\hline $8 \mathrm{~K}$ & 0.654 & 0.645 & 0.622 & 0.598 & 0.627 & 0.667 \\
\hline $16 \mathrm{~K}$ & 0.627 & 0.616 & 0.609 & 0.581 & 0.633 & 0.646 \\
\hline $32 \mathrm{~K}$ & 0.632 & 0.601 & 0.559 & 0.581 & 0.615 & 0.646 \\
\hline \multicolumn{7}{|c|}{ 64B:32B } \\
\hline & \multicolumn{2}{|c|}{ Unified } & \multicolumn{2}{|c|}{ Instruction } & \multicolumn{2}{|c|}{ Data } \\
\hline Size & Media & [Smit87] & Media & [Smit87] & Media & [Smit87] \\
\hline $2 \mathrm{~K}$ & 0.819 & 0.787 & 0.698 & 0.701 & 0.889 & 0.853 \\
\hline $4 \mathrm{~K}$ & 0.785 & 0.746 & 0.831 & 0.660 & 0.740 & 0.770 \\
\hline $8 \mathrm{~K}$ & 0.756 & 0.661 & 0.717 & 0.667 & 0.706 & 0.723 \\
\hline $16 \mathrm{~K}$ & 0.676 & 0.633 & 0.633 & 0.624 & 0.662 & 0.672 \\
\hline $32 \mathrm{~K}$ & 0.656 & 0.601 & 0.696 & 0.624 & 0.686 & 0.662 \\
\hline \multicolumn{7}{|c|}{ 128B:64B } \\
\hline & \multicolumn{2}{|c|}{ Unified } & \multicolumn{2}{|c|}{ Instruction } & \multicolumn{2}{|c|}{ Data } \\
\hline Size & Media & [Smit87] & Media & [Smit87] & Media & [Smit87] \\
\hline $4 \mathrm{~K}$ & 0.938 & 1.809 & 0.733 & 0.831 & 0.925 & 0.835 \\
\hline $8 \mathrm{~K}$ & 0.937 & 0.753 & 0.776 & 0.690 & 0.865 & 0.817 \\
\hline $16 \mathrm{~K}$ & 0.868 & 0.685 & 0.655 & 0.657 & 0.841 & 0.747 \\
\hline $32 \mathrm{~K}$ & 0.739 & 0.660 & 0.854 & 0.634 & 0.750 & 0.697 \\
\hline
\end{tabular}

Table 5: Comparison to [Smit87] - Ratio of miss ratio to that of line half as long. Results are shown only for those cache configurations that are common to both [Smit87] and this study.

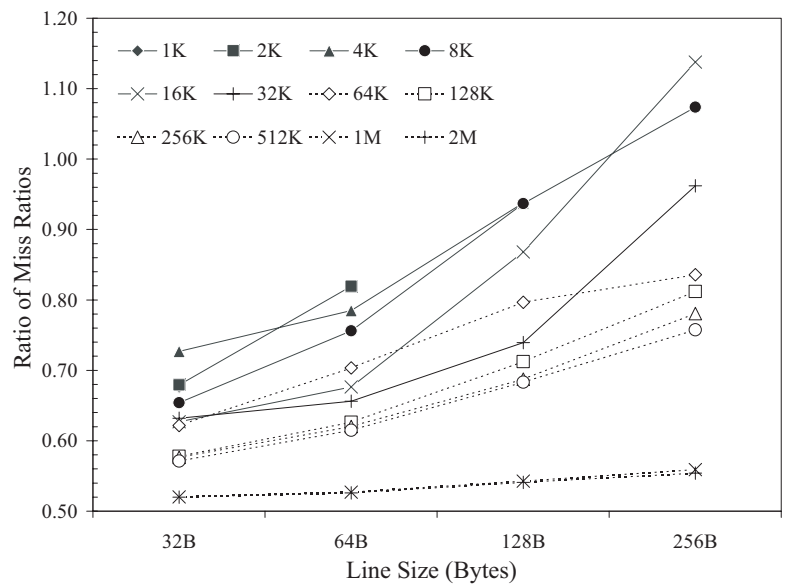

Figure 3: Ratio of Miss Ratios: Unified Cache - Berkeley Multimedia Workload

to overall miss ratio. Figure 6 graphs the variation of the three types of misses for MPEG decoding at DVD resolution, and the SPECint95 147.vortex application. What is clear is that these two applications have widely different proportions of conflict, capacity and compulsory miss ratios for any given cache capacity. Although individual traces exhibit widely differing contributions from the three miss ratio factors, traces

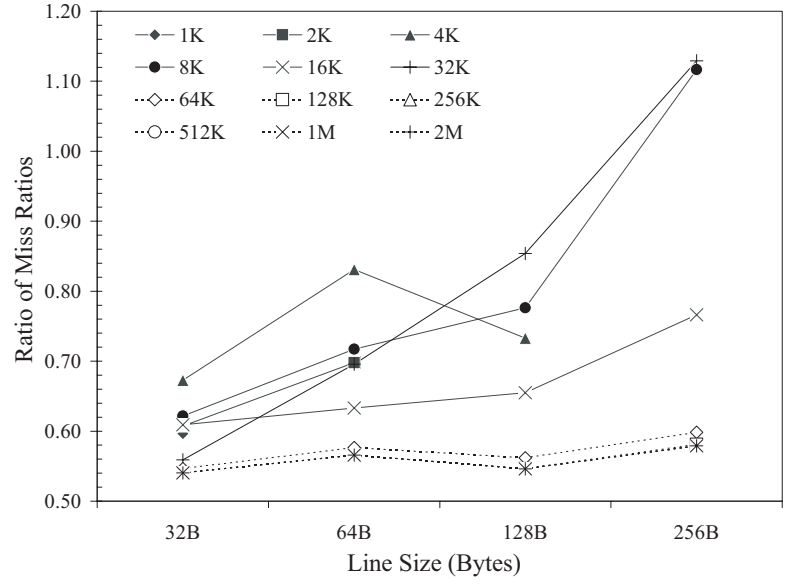

(a) Instruction Cache

Figure 4: Ratio of Miss Ratios: Instruction Cache Berkeley Multimedia Workload

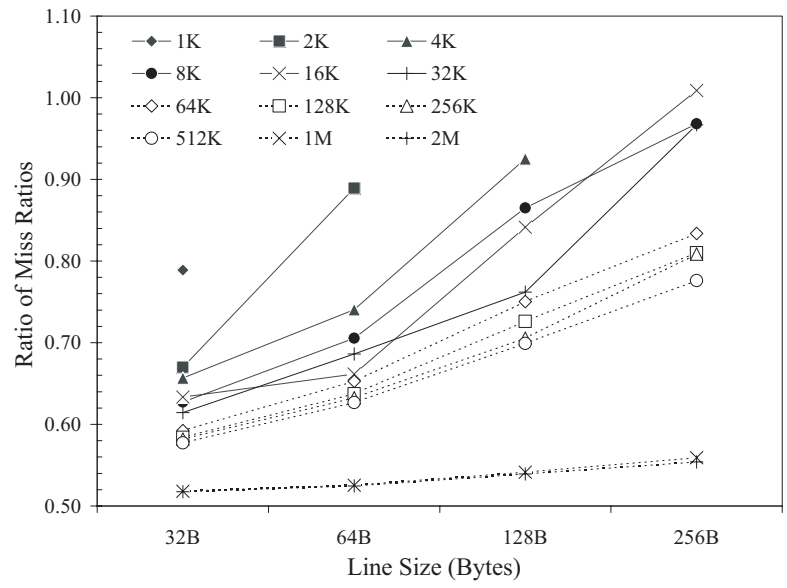

(a) Data Cache

Figure 5: Ratio of Miss Ratios: Data Cache - Berkeley Multimedia Workload

on average follow the heuristics of [Hill89] (see Figure 7).

\subsection{Workloads}

\subsubsection{SPEC92 [Gee93]}

In the past decade, SPEC (Standard Performance Evaluation Corporation) benchmarks have played an extremely important role in both hardware and compiler design. SPEC CPU benchmarks are taken to be generally representative of traditional workstation applications, with the integer component reflecting system or commercial applications, and the floating point component representing numeric and scientific applications. The SPEC92 benchmark consists of six integer- 


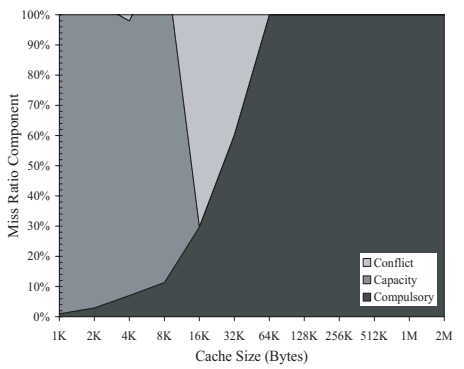

(a) DVD - Instruction Cache

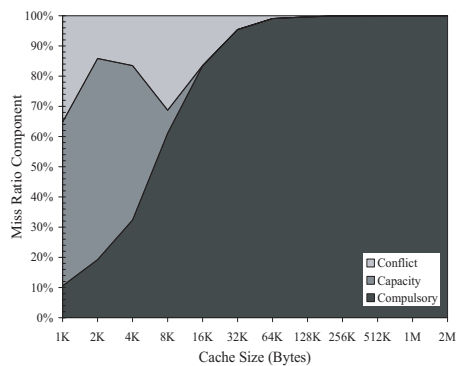

(b) DVD - Data Cache

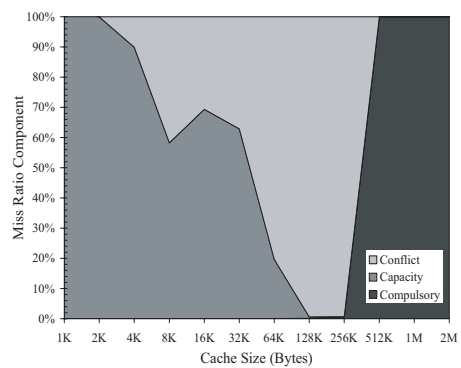

(c) Vortex - Instruction Cache

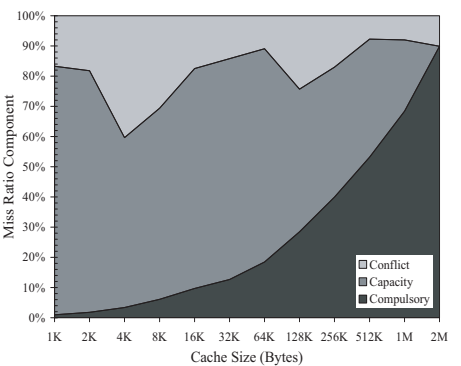

(d) Vortex - Data Cache

Figure 6: Miss Ratio Factors

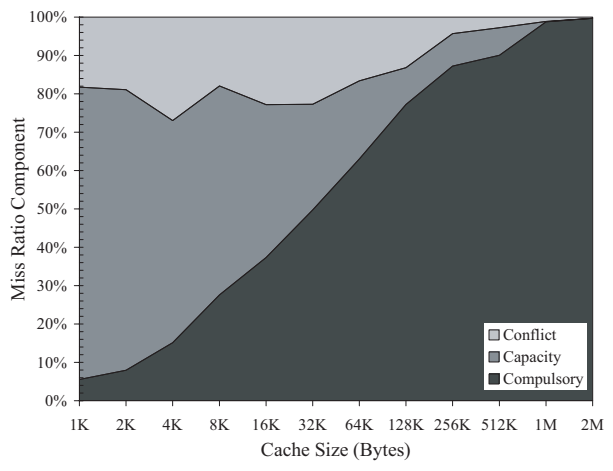

(a) Multimedia - Unified Cache

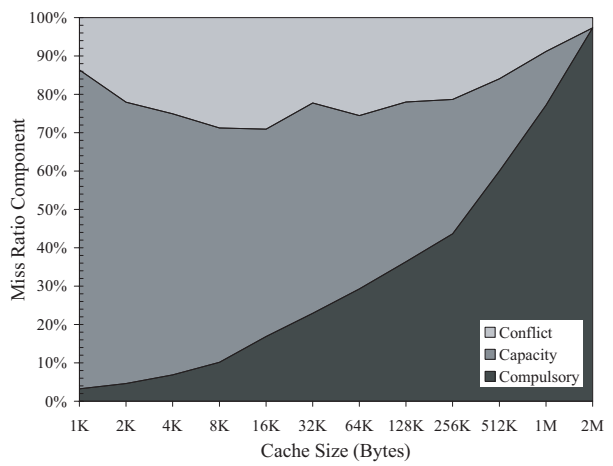

(d) SPEC95 - Unified Cache

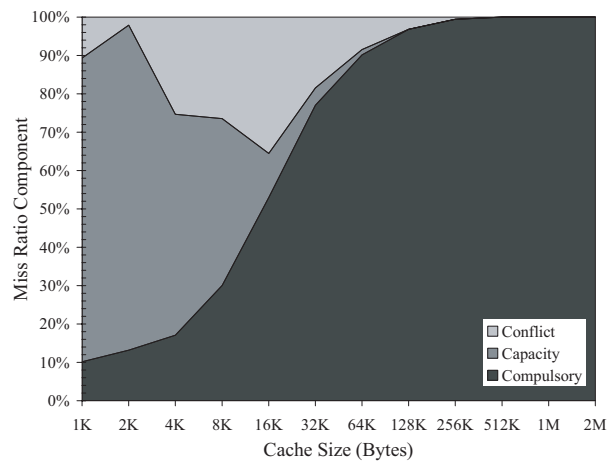

(b) Multimedia - Instruction Cache

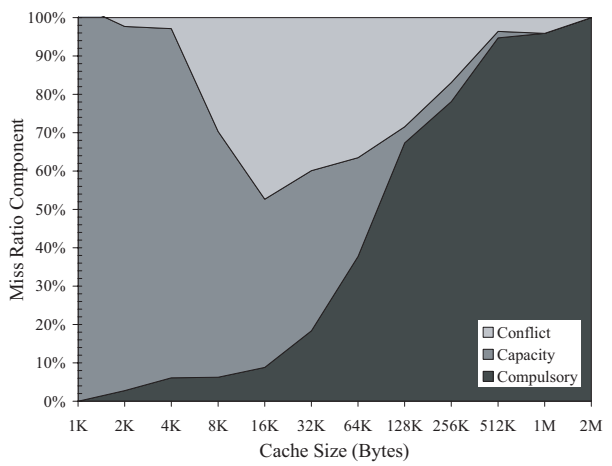

(e) SPEC95 - Instruction Cache

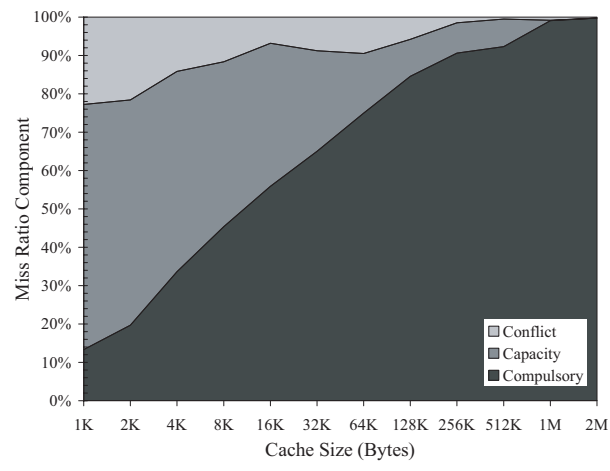

(c) Multimedia - Data Cache

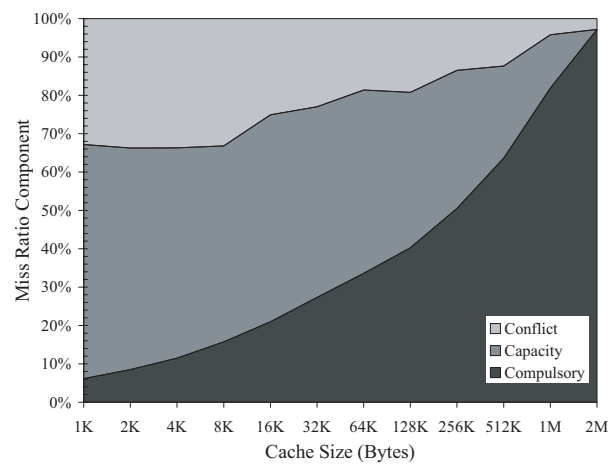

(f) SPEC95 - Data Cache

Figure 7: Average Miss Ratio Factors

intensive, and fourteen floating-point-intensive programs (see Table 6). All of the component benchmarks are written in either C (integer) or Fortran (floating point).

It is important to note that although the SPEC component applications are based on real world counterparts, many of them were modified by SPEC from their original form to reduce the influence of $\mathrm{I} / \mathrm{O}$ and the operating system on performance. In some instances, this means that test data is read from and written to memory, rather than disk (as would be the case in the original form of the application). Clearly this could potentially affect memory behavior compared to real world applications.

[Gee93] analyzed the cache behavior of the SPEC92 benchmark suite running on DECstations with MIPS R2000 or R3000 processors and version 4.1 of the DEC Ultrix operating system. Version 2.0 of the $\mathrm{C}$ compiler and version 2.1 of the FORTRAN compiler were used with the optimization level according to the SPEC Makefiles. Because the SPEC benchmarks are typically run in a uniprogrammed environment, no cache flushing or other method was used to simulate 
multiprogramming. [Gee93] also found that for the SPEC92 benchmark suite, system time is insignificant compared to user time, and so operating system memory behavior is largely unimportant for this suite of applications.

\begin{tabular}{|c|c|}
\hline Integer & Description \\
\hline 008.espresso & Circuit theory \\
\hline $022.1 \mathrm{i}$ & Lisp interpreter \\
\hline 023.eqnitott & Logic design \\
\hline 026.compress & Lempel-Ziv coding \\
\hline $072 . s c$ & Spread sheet \\
\hline $085 . \mathrm{gcc}$ & GNU C Compiler \\
\hline Floating Point & Description \\
\hline 013.spice2g6 & Circuit design \\
\hline 015.doduc & Monte-Carlo simulation \\
\hline 034.mdljdp2 & Quantum chemistry \\
\hline 039.wave5 & Maxwell's equations \\
\hline 047.tomcatv & Coordinate translation \\
\hline 048.ora & Optics ray-tracing \\
\hline 052.alvinn & Robotics \\
\hline 056. ear & Model of the human ear \\
\hline 077.mdljsp2 & Quantum chemistry \\
\hline 078. swm256 & Shallow water model \\
\hline 089.su2cor & Quantum physics \\
\hline 090.hyrdo2d & Astrophysics \\
\hline 093.nasa7 & NASA kernels \\
\hline 094.fpppp & Quantum chemistry \\
\hline
\end{tabular}

Table 6: SPEC92 Benchmark Suite

\subsubsection{SPEC95}

SPEC95 is an upgraded version of the SPEC92 benchmark suite. It consists of eight integer intensive and ten floatingpoint intensive applications. Several of the programs from SPEC92 are found in SPEC95. 126. gcc is the same as 085.gcc except that a newer version of gcc is used. 129.compress is the same as 026.compress. $130.1 \mathrm{i}$ is the same as 022.li. 101.tomcatv is the same as 047.tomcatv. 102.swim is the same as 078. swm 256 except that it uses a $1024 \times 1024$ grid. 103 .su2cor is the same as 089.su2cor. 104.hydro2d is the same as 090.hydro2d. 145.fpppp is the same as 094.fpppp. 146.wave5 is the same as 039.wave5. In general, the applications were designed to have larger code size and greater memory activity than those of SPEC92.

\subsubsection{Multiprogramming Workload (Mult) [Borg90]}

[Borg90] generated miss ratios for very long address traces (up to 12 billion memory references in length) on the Titan RISC architecture in order to evaluate the performance of a variety of cache designs. Three individual traces were used in addition to another which was a multiprogrammed workload consisting of several jobs. Our comparison includes their miss ratio results for their 7.6 billion reference $(68.5 \%$ instruction, $30.6 \%$ load, $15.4 \%$ store) multiprogramming workload (referred to as "Mult" by the authors of [Borg90]). It consists of:

- make run compiling (from C) portions of the magic source code

- grr routing the DECstation 3100 printed circuit board

- magic (VLSI editor) design rule checking the MultiTitan CPU chip

- tree (compiled Scheme) builds tree data structure and searches for largest element

- make run that calls xld (linker/loader) on the magic code

- tcsh script of infinite loop of interactive commands (cp, cat, ex, rm, ps, -aux, ls $-1 / *$ )

\subsubsection{Design Target Miss Ratios (DTMR) [Smit85]}

[Smit85] introduced the concept of design target miss ratios (DTMRs), intended to represent typical levels of performance across a wide class of workloads and machines, to be used for hardware design. The DTMRs were synthesized from real (hardware monitor) measurements that existed in the literature and from trace driven simulations using a large number of traces taken from several architectures, originally coded in several different languages.

\subsubsection{VAX 11/780 [Clark83], VAX 8800 [Clark88]}

Two studies done at Digital Equipment Corporation (DEC) supply miss ratios for a time-shared engineering workload taken with a hardware monitor on VAX $11 / 780$ and VAX 8800 machines [Clark83], [Clark88]. The 11/780 has an 8-KB, write through, unified cache with an 8-byte block size and associativity of two. The 8800 has a $64-\mathrm{KB}$, write-through, direct mapped, unified cache with a 64 -byte block size. On the VAX $11 / 780$ it is possible to disable half of the two-way associative cache through special control bits; a technique which allowed for measurement of a $4-\mathrm{KB}$, direct mapped, unified cache configuration as well.

\subsubsection{Agarwall Mul3 [Agar88]}

In [Agar88] an analysis of the effect of operating system references and multiprogramming was presented for a workload of eleven application programs (30 traces in all). The platform used to gather the traces was a VAX 11/780 running either the Ultrix or VMS operating system. All of the traces were gathered through the ATUM scheme of microcode modification, and were roughly 400,000 references long (approximately half a second of execution time). A technique termed trace sampling was used to concatenate smaller traces to better simulate the full trace length of a running program. We utilize their three way multiprogrammed workload for comparison. 


\begin{tabular}{|c|c|c|c|c|}
\hline Integer & Description & Instruction & Load & Store \\
\hline 099.go & AI plays "Go" & $32,465,547,697$ & $8,168,233,239$ & $2,409,111,305$ \\
\hline $124 . \mathrm{m} 88 \mathrm{ksim}$ & Motorola $88 \mathrm{~K}$ simulator & $73,820,020,885$ & $13,680,509,709$ & $7,402,458,586$ \\
\hline 126.gcc & GCC, builds SPARC code & $209,948,942$ & $49,135,874$ & $24,970,849$ \\
\hline 129.compress & Lempel-Ziv coding & $60,850,564,695$ & $11,722,190,689$ & $4,458,902,946$ \\
\hline $130.1 \mathrm{i}$ & Lisp interpreter & $65,730,724,167$ & $16,730,950,984$ & $9,385,736,837$ \\
\hline 132.ijpeg & DCT image compression & $34,116,845,423$ & $6,023,880,310$ & $1,870,937,674$ \\
\hline 134.perl & Anagrams/primes in Perl & $29,378,239,506$ & $6,868,627,321$ & $4,173,646,937$ \\
\hline 147.vortex & Database & $87,529,422,776$ & $20,621,987,100$ & $11,989,029,595$ \\
\hline Floating Point & Description & & & \\
\hline 101.tomcatv & Mesh generation & $31,932,871,503$ & $6,481,320,753$ & $3,324,212,603$ \\
\hline 102.swim & Shallow water model & $32,575,361,567$ & $5,702,422,493$ & $2,773,604,570$ \\
\hline 103.su2cor & Monte Carlo simulation & $39,599,980,228$ & $7,061,086,677$ & $3,214,956,672$ \\
\hline 104.hydro2d & Hydrodynamical Eqns & $48,836,577,610$ & $10,074,625,107$ & $3,468,630,651$ \\
\hline 107.mgrid & Multigrid solver in 3D field & $72,429,596,913$ & $18,187,911,190$ & $3,911,599,079$ \\
\hline 110.applu & Differential equations & $33,716,422,315$ & $7,722,618,047$ & $3,267,428,916$ \\
\hline 125.turb3d & Turbulence in cube & $104,351,000,000$ & $15,919,035,949$ & $13,253,927,515$ \\
\hline 141.apsi & Temp, Wind, Potential & $33,155,694,301$ & $6,254,642,763$ & $3,445,287,831$ \\
\hline 145.fpppp & Quantum chemistry & $147,327,000,000$ & $43,911,029,435$ & $11,283,084,727$ \\
\hline 146. wave5 & Plasma physics & $34,209,423,864$ & $6,831,165,556$ & $4,455,048,459$ \\
\hline Total & & $962,235,242,392$ & $212,011,373,196$ & $94,112,575,752$ \\
\hline Arithmetic Mean & & $53,457,513,466$ & $11,778,409,622$ & $5,228,476,431$ \\
\hline
\end{tabular}

Table 7: SPEC95 Benchmark Suite Characteristics

\subsubsection{Amdahl 470 [Smit82]}

In [Smit82], hardware monitor measurements taken at Amdahl Corporation on Amdahl 470V machines are presented. A standard internal benchmark was run containing supervisor, commercial and scientific code. Supervisor state miss ratios were found to be much higher than problem state miss ratios.

\section{References}

[Agar88] Anant Agarwal, John Hennessy, Mark Horowitz, "Cache Performance of Operating System and Multiprogramming Workloads," ACM Trans. on Computer Systems, Vol. 6, No. 4, November 1988, pp. 393-431

[AMD] Advanced Micro Devices, Inc., "AMD Athlon Processor x86 Code Optimization Guide," Publication \#22007G/0, April 2000, http://www.amd.com/products/cpg/athlon/techdocs/pdf/ 2200\%.pdf, retrieved April 24, 2000

[Borg90] Anita Borg, R. E. Kessler, David W. Wall, "Generation and Analysis of Very Long Address Traces," Proc. 17th Int'l Symp. on Computer Architecture, Seattle, Washington, May 28-31, 1990, pp. $270-279$

[Brow97] A. Brown, and M. Seltzer. "Operating System Benchmarking in the Wake of Lmbench: A Case Study of the Performance of NetBSD on the Intel x86 Architecture." Proc. 1997 ACM SIGMETRICS Conference on Measurement and Modeling of Computer Systems, Seattle, Washington, June 15-18, 1997, pp. 214-224

[Burd] Tom Burd, "CPU Info Center: General Processor Info," http://bwrc.eecs.berkeley.edu/CIC/summary/local/summary.pdf, retrieved April 24, 2000

[Clark83] D. W. Clark, "Cache Performance in the VAX-11/780," ACM Trans. on Computing Systems, Vol. 1, No. 1, February 1983 pp. $24-37$

[Clark88] D. W. Clark, P. J. Bannon, J. B. Keller, "Measuring VAX 8800 Performance with a Histogram Hardware Monitor," Proc. 15th Int'l Symp. on Computer Architecture, Honolulu, Hawii, May 30-June 2, 1988, pp. 176-185
[Gee93] Jeffrey D. Gee, Mark D. Hill, Dionisios N. Pnevmatikatos, Alan Jay Smith, "Cache Performance of the SPEC92 Benchmark Suite," IEEE Micro, Vol. 13, No. 4, August 1993, pp. 17-27

[Hill87] Mark D. Hill, "Aspects of Cache Memory and Instruction Buffer Performance," Ph.D. Thesis, University of California at Berkeley, Computer Science Division, Technical Report UCB/CSD $87 / 381$ (November, 1987)

[Hill89] Mark D. Hill, Alan Jay Smith, "Evaluating Associativity in CPU Caches," IEEE Trans. on Computers, Vol. 38, No. 12, December 1989 , pp. $1612-1630$

[Koba86] Makoto Kobayashi, "An Empirical Study of Task Switching Locality in MVS," IEEE Transactions on Computers, Vol. C-35, No. 8, August 1986 , pp. $720-731$

[McVoy96] Larry McVoy, Carl Staelin, "lmbench: Portable tools for performance analysis," Proc. of Winter 1996 USENIX Conference, San Diego, California, January 22-26, 1996, pg 279-294

[Noer] Kim Noer, "Heat Dissipation Per Square Millimeter Die Size Specifications," http://home.worldonline.dk/ ${ }^{\sim}$ noer/, retrieved April 24, 2000

[Pett] Mikael Pettersson, "Perfect Counters Home Page," http://www.csd.uu.se/ mikpe/linux/perfctr, retrieved April 24,2000

[Saav95] Rafael H. Saavedra, Alan Jay Smith, "Measuring Cache and TLB Performance and Their Effect on Benchmark Runtimes," IEEE Trans. on Computers, Vol. 44 No. 10, October 1995, pp. 12231235

[Smit82] Alan Jay Smith, "Cache Memories," ACM Computing Surveys, Vol. 14, No. 3, September 1982, pp. 473-530

[Smit85] Alan Jay Smith, "Cache Evaluation and the Impact of Workload Choice," Proc. 12th Int'l Symp. on Computer Architecture, Boston, Massachusetts, June 17-19, 1985, pp. 64-73

[Smit87] Alan Jay Smith, "Line (Block) Size Choice for CPU Cache Memories," IEEE Trans. on Computers, Vol. C-36, No. 9, September 1987, pp. 1063-1075 
Berkeley Multimedia Workload

\begin{tabular}{|c|c|c|c|c|c|}
\hline & & & ultimedi & & \\
\hline & & $\frac{\omega}{\text { Instr }}$ & unction & ache & \\
\hline & & & ociativi & & \\
\hline & & & & 8-way & $6-w a y$ \\
\hline & & $\begin{array}{l}.05594 \\
02815 \\
2815\end{array}$ & 0.05259 & 0.05103 & \\
\hline & & $\begin{array}{l}.02815 \\
0.01506\end{array}$ & 0.0 & $\begin{array}{l}.02622 \\
0.01140\end{array}$ & $\begin{array}{l}64 \\
46\end{array}$ \\
\hline $8 \mathrm{~F}$ & & & & & \\
\hline & & & & & \\
\hline $64 \mathrm{~K}$ & 0.0 & & & 33 & \\
\hline & & & & & \\
\hline & & & & 0 & \\
\hline & & & & & \\
\hline & & 0.0 & & 0.0 & \\
\hline & & 0.00067 & 0.00067 & 0.00067 & .00067 \\
\hline & & & & & \\
\hline & 0.12894 & 19] & & 325 & \\
\hline $\begin{array}{l}2 \mathrm{~K} \\
4 \mathrm{~K}\end{array}$ & & & & 60 & \\
\hline & & & & & \\
\hline & & & & & \\
\hline & & & & & \\
\hline & & & & & \\
\hline & & & & & \\
\hline 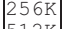 & & & & & \\
\hline 121 & & & 0.0 & 0.0 & \\
\hline & & & & 0.0 & \\
\hline & & & & & \\
\hline & & & & 09 & \\
\hline & & & & & \\
\hline & & & & & \\
\hline & & & & & \\
\hline & $\begin{array}{l}0.0 \\
0.0\end{array}$ & & $\begin{array}{l}0.0 \\
0.0\end{array}$ & & \\
\hline & & & & & \\
\hline & & & & & \\
\hline & & & & & \\
\hline & & & & & \\
\hline & & & & & \\
\hline
\end{tabular}

\begin{tabular}{|c|c|c|c|c|c|}
\hline & & & Document & & \\
\hline & & & uction & & \\
\hline$\overline{z e}$ & Direct & & 4-way & & 16-way \\
\hline $1 \mathrm{~K}$ & 0.08120 & 0.08365 & \begin{tabular}{|l|}
0.08882 \\
\end{tabular} & 0.08198 & 0.08455 \\
\hline & $\begin{array}{l}0.05397 \\
0.03315\end{array}$ & $\begin{array}{l}0.04785 \\
0.02371\end{array}$ & $\left|\begin{array}{l}0.04111 \\
0.01935\end{array}\right|$ & $\begin{array}{l}0.04335 \\
0.01118\end{array}$ & $\begin{array}{l}0.04540 \\
0.005880\end{array}$ \\
\hline & 0.01968 & 0.01183 & 0.00607 & 0.00372 & 0.00083 \\
\hline & 0.00818 & 0.00361 & 0.00151 & 0.00049 & 0.00047 \\
\hline & 0.00592 & 0.00044 & 0.00038 & 0.00033 & 0.00034 \\
\hline & 0.00572 & 0.00028 & 0.00023 & 0.00021 & 0.00020 \\
\hline $\begin{array}{l}128 \mathrm{~K} \\
256 \mathrm{~K}\end{array}$ & $\begin{array}{l}0.00215 \\
0.00193\end{array}$ & $\begin{array}{l}0.00019 \\
0.00018\end{array}$ & $\begin{array}{l}0.00018 \\
0.00018\end{array}$ & $\begin{array}{l}0.00018 \\
0.00018\end{array}$ & $\begin{array}{l}0.00018 \\
0.00018\end{array}$ \\
\hline & 0.00018 & 0.00018 & 0.00018 & 0.00018 & 0.00018 \\
\hline & 0.00018 & 0.00018 & 0.00018 & 0.00018 & 0.00018 \\
\hline & 0.00018 & 0.00018 & 0.00018 & 0.00018 & 0.00018 \\
\hline & & & ta & & \\
\hline & 0.16031 & $\frac{\text { z-way }}{0.11569}$ & \begin{tabular}{|c|} 
4-way \\
0.09283 \\
\end{tabular} & 0.08694 & $\frac{10 \text { wady }}{0.08255}$ \\
\hline $\begin{array}{l}2 \mathrm{~K} \\
4 \mathrm{~K}\end{array}$ & 0.10863 & $\begin{array}{l}0.07608 \\
0.05406\end{array}$ & 0.05455 & $\begin{array}{r}0.04709 \\
0.03988\end{array}$ & $\begin{array}{l}0.04573 \\
0.03948\end{array}$ \\
\hline & 0.04902 & 0.03662 & 0.03549 & 0.03413 & 0.03393 \\
\hline & & & 0.02937 & 0.02936 & \\
\hline & 0.03260 & 0.02730 & 0.02625 & 0.02609 & 0.02608 \\
\hline & $\begin{array}{l}0.03016 \\
0.02514\end{array}$ & $\left.\begin{array}{l}0.02526 \\
0.02471\end{array}\right]$ & $\begin{array}{r}0.02485 \\
0.02453\end{array}$ & $\begin{array}{r}0.02475 \\
0.02452\end{array}$ & 0.02472 \\
\hline $56 \mathrm{~K}$ & 0.02464 & 0.02435 & 0.02434 & 0.02434 & 0.02434 \\
\hline $12 \mathrm{~K}$ & 0.01 & 0.02297 & 0.02428 & 0.02429 & 0.02 \\
\hline $1 \mathrm{M}$ & 0.01278 & 0.01266 & 0.01265 & 0.01265 & 0.01265 \\
\hline & 0.01264 & & & 0.01263 & 0.01263 \\
\hline & & & & & \\
\hline & & & $\begin{array}{l}\frac{4-w a y}{0.12367} \\
\end{array}$ & 0.12248 & 521 \\
\hline & $\begin{array}{r}.10053 \\
.06529\end{array}$ & $\left.\begin{array}{l}0.08617 \\
0.04708\end{array}\right]$ & $\begin{array}{l}0.07801 \\
0.03836\end{array}$ & $\begin{array}{l}0.07543 \\
0.03235\end{array}$ & $\begin{array}{l}0.07352 \\
0.03137\end{array}$ \\
\hline & 091 & 0002 & 0.01541 & 0.01244 & \\
\hline & 2 & 0. & 0.008 & (200714 & \\
\hline & & & 0.05050 & 0.00504 & \\
\hline & .01324 & $\begin{array}{l}0.00 \\
0.00\end{array}$ & $\begin{array}{l}0.00550 \\
0.00525\end{array}$ & $\begin{array}{l}0.00544 \\
0.00522\end{array}$ & $\begin{array}{l}0.06 \\
0.08\end{array}$ \\
\hline & & & 0.00516 & 0.0 & \\
\hline & 04 & 0.00 & $\begin{array}{l}0.00515 \\
0.00273\end{array}$ & $\begin{array}{l}0.00515 \\
0.00273\end{array}$ & \\
\hline & & & & & \\
\hline
\end{tabular}

Audio
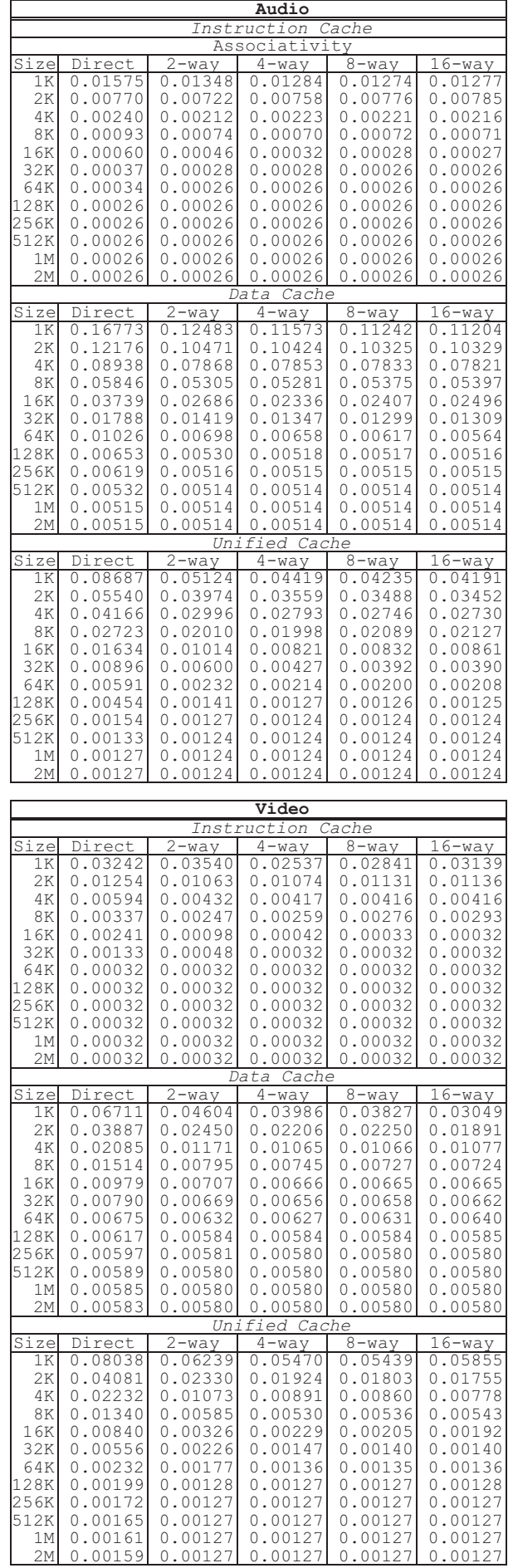

Block Size: 16 bytes Speech

\begin{tabular}{|c|c|c|c|c|c|}
\hline \\
\hline \multirow{2}{*}{\multicolumn{6}{|c|}{$\begin{array}{c}\text { Instruction Cache } \\
\text { Associativity }\end{array}$}} \\
\hline & & & & & \\
\hline$i z e$ & Direct & 2-way & 4-way & 8-way & 16-way \\
\hline $1 \mathrm{~K}$ & 0.08537 & 0.08410 & 0.07580 & 0.06868 & 0.06710 \\
\hline $2 \mathrm{~K}$ & 0.03110 & 0.03111 & 0.02533 & 0.02497 & 0.02509 \\
\hline $4 \mathrm{~K}$ & 0.02261 & 0.01934 & 0.01925 & 0.02011 & 0.02117 \\
\hline $8 \mathrm{~K}$ & 0.01311 & 0.01093 & 0.00856 & 0.00726 & 0.00698 \\
\hline $16 \mathrm{~K}$ & 0.00773 & 0.00534 & 0.00434 & 0.00460 & 0.00469 \\
\hline $32 \mathrm{~K}$ & 0.00387 & 0.00292 & 0.00256 & 0.00222 & 0.00213 \\
\hline $64 \mathrm{~K}$ & 0.00312 & 0.00222 & 0.00215 & 0.00210 & 0.00210 \\
\hline $128 \mathrm{~K}$ & 0.00236 & 0.00215 & 0.00210 & 0.00210 & 0.00210 \\
\hline $256 \mathrm{~K}$ & 0.00225 & 0.00210 & 0.00210 & 0.00210 & 0.00210 \\
\hline $512 \mathrm{~K}$ & 0.00210 & 0.0 & 0.00210 & 0.00210 & 0.00210 \\
\hline $1 \mathrm{M}$ & 0.00210 & 0.00210 & 0.00210 & 0.00210 & 0.00210 \\
\hline $2 \mathrm{M}$ & 0.00210 & 0.00210 & 0.00210 & 0.00210 & 0.00210 \\
\hline \multicolumn{6}{|c|}{ Data Cache } \\
\hline$z e$ & Direct & 2-way & 4-way & 8-way & 6-way \\
\hline $1 \mathrm{~K}$ & 0.07101 & 0.03968 & 0.03498 & 0.03365 & 0.03282 \\
\hline $2 \mathrm{~K}$ & 0.04576 & 0.02355 & 0.02034 & 0.01958 & 0.01915 \\
\hline $4 \mathrm{~K}$ & 0.02472 & 0.01564 & 0.01433 & 0.01336 & 0.01323 \\
\hline $8 \mathrm{~K}$ & 0.01810 & 0.01306 & 0.01203 & 0.01196 & 0.01194 \\
\hline $16 \mathrm{~K}$ & 0.01261 & 0.01059 & 0.01052 & 0.01107 & 0.01139 \\
\hline $32 \mathrm{~K}$ & 0.00831 & 0.00670 & 0.00645 & 0.00565 & 0.00534 \\
\hline $64 \mathrm{~K}$ & 0.00646 & 0.0 & 0.00444 & 0.00432 & 0.00428 \\
\hline $8 \mathrm{~K}$ & 0.00502 & 0.00460 & 0.00425 & 0.00423 & 0.00423 \\
\hline $56 \mathrm{~K}$ & 0.00434 & 0.00423 & 0.00422 & 0.00422 & 0.00422 \\
\hline $12 \mathrm{~K}$ & 0.00424 & 0.0 & 0.00422 & 0.004 & 0.00422 \\
\hline $1 \mathrm{M}$ & 0.00422 & 0.00422 & 0.00422 & 0.00422 & 0.00422 \\
\hline $2 \mathrm{M}$ & 0.00422 & 0.00422 & 0.00422 & 0.00422 & 0.00422 \\
\hline \multicolumn{6}{|c|}{ fied $\mathrm{Cac}$} \\
\hline $\mathrm{ze}$ & Dir & & 4-way & $8-w a$ & \\
\hline $1 \mathrm{~K}$ & 0.13689 & 0.10809 & 0.10562 & 0.10710 & \\
\hline $2 \mathrm{~K}$ & 0.08654 & 0.06040 & 0.06637 & 0.06477 & 0.06461 \\
\hline $4 \mathrm{~K}$ & 0.05718 & 0.02865 & 0.02468 & 0.02439 & 0.02443 \\
\hline $8 \mathrm{~K}$ & 0.03182 & 0.01647 & 0.01357 & 0.01323 & 0.01254 \\
\hline $16 \mathrm{~K}$ & 0.02403 & 0.00965 & 0.00753 & 0.00674 & 0.00668 \\
\hline $32 \mathrm{~K}$ & 0.01900 & 0.0 & 0.00579 & 0.00586 & 0.00613 \\
\hline $64 \mathrm{~K}$ & 0.01583 & 0.00390 & 0.00329 & 0.00323 & 0.00280 \\
\hline $128 \mathrm{~K}$ & 0.00421 & 0.00306 & 0.00263 & 0.00255 & 0.00255 \\
\hline $256 \mathrm{~K}$ & 0.00379 & 0.00257 & 0.00255 & 0.00255 & 0.00255 \\
\hline $512 \mathrm{~K}$ & 0.00286 & 0.00255 & 0.00255 & 0.00255 & 0.00255 \\
\hline $1 \mathrm{M}$ & 0.00286 & 0.00255 & 0.00255 & 0.00255 & 0.00255 \\
\hline $2 \mathrm{M}$ & 0.00286 & 0.00255 & 0.00255 & 0.00255 & 0.00255 \\
\hline
\end{tabular}

3D

\begin{tabular}{|c|c|c|c|c|c|}
\hline & & & & & \\
\hline & & Instr & tion $C$ & ache & \\
\hline 12 & irec & 2-way & $4-w a y$ & 8-way & 16-way \\
\hline $1 \mathrm{~K}$ & 0.06637 & 0.06305 & 0.06410 & 0.06333 & 0.06319 \\
\hline $2 \mathrm{~K}$ & 0.04316 & 0.04392 & 0.04329 & 0.04372 & 0.04351 \\
\hline $4 \mathrm{~K}$ & 0.02842 & 0.02580 & 0.02079 & 0.01933 & 0.01901 \\
\hline $8 \mathrm{~K}$ & 0.01929 & 0.01706 & 0.01548 & 0.01544 & 0.01572 \\
\hline $16 \mathrm{~K}$ & 0.01294 & 0.00914 & 0.00834 & 0.00830 & 0.00849 \\
\hline $32 \mathrm{~K}$ & 0.00734 & 0.00341 & 0.00242 & 0.00149 & 0.00069 \\
\hline $64 K$ & 0.00419 & 0.00136 & 0.00075 & 0.00055 & 0.00052 \\
\hline $128 \mathrm{~K}$ & 0.00305 & 0.00054 & 0.00051 & 0.00051 & 0.00051 \\
\hline $256 \mathrm{~K}$ & 0.00165 & 0.00051 & 0.00051 & 0.00051 & 0.00051 \\
\hline $512 \mathrm{~K}$ & 0.00097 & 0.00051 & 0.00051 & 0.00051 & 0.00051 \\
\hline $1 \mathrm{M}$ & 0.0 & 0.00051 & 0.00051 & 0.00051 & 0.00051 \\
\hline $2 \mathrm{M}$ & 0. & 0.00 & 0.00051 & 0.00051 & 0.00051 \\
\hline & & & ta & & \\
\hline $\mathrm{ze}$ & D & 2 & 4-way & $8-w$ & 1 \\
\hline $1 \mathrm{~K}$ & 0.17853 & 0.15471 & 0.14860 & 0.14498 & 0.14304 \\
\hline $2 \mathrm{~K}$ & 0.1 & 0.1 & 0.12752 & 0.1 & 0.12510 \\
\hline $4 \mathrm{~K}$ & 0.1 & 0.11642 & 0.11336 & 0.11320 & 0.11340 \\
\hline $8 \mathrm{~K}$ & 0.10442 & 0.09812 & 0.09681 & 0.09629 & 0.09627 \\
\hline $16 \mathrm{~K}$ & 0.09048 & 0.08186 & 0.07982 & 0.07866 & 0.07768 \\
\hline $32 \mathrm{~K}$ & 0.07952 & 0.07146 & 0.06886 & 0.06774 & 0.06710 \\
\hline $64 \mathrm{~K}$ & 0.0 & 0.0 & 0.06442 & 0.06403 & 0. \\
\hline $128 \mathrm{~K}$ & 0.06482 & 0.06325 & 0.06253 & 0.06257 & 0.06262 \\
\hline $256 \mathrm{~K}$ & 0.06283 & 0.06098 & 0.06061 & 0.06049 & 0.06046 \\
\hline $\mathrm{K}$ & & 0.0 & 0.0 & 0.0 & 0.05961 \\
\hline $1 \mathrm{M}$ & 0.0 & 0.0 & 0.0 & 0.05 & 0.05903 \\
\hline $2 \mathrm{M}$ & 0. & 0. & 0.05883 & 0.05883 & 0.05883 \\
\hline & & & ied & & \\
\hline ize & Dir & $2-1$ & $4-w$ & 8-way & 16-way \\
\hline $1 \mathrm{~K}$ & 0.13496 & 0.10979 & 0.10491 & 0.10415 & 0.10486 \\
\hline $2 \mathrm{~K}$ & 0.10649 & 0.08525 & 0.08300 & 0.08253 & 0.08234 \\
\hline $4 \mathrm{~K}$ & 0.0 & 0.06089 & 0.05571 & 0.05288 & 0.05213 \\
\hline $8 \mathrm{~K}$ & 0.05201 & 0.04439 & 0.04191 & 0.04065 & 0.04070 \\
\hline $16 \mathrm{~K}$ & 0.0 & 0.03473 & 0.03373 & 0.03313 & 0.03283 \\
\hline $32 \mathrm{~K}$ & 0.03215 & 0.02661 & 0.02442 & 0.02418 & 0.02449 \\
\hline $64 K$ & 0.02542 & 0.02016 & 0.01722 & 0.01609 & 0.01563 \\
\hline $128 \mathrm{~K}$ & 0.02046 & 0.01632 & 0.01504 & 0.01479 & 0.01478 \\
\hline & & 0.0 & 0.01429 & 0.01426 & 0.01426 \\
\hline $512 \mathrm{~K}$ & 0. & 0.01427 & 0.01392 & 0.01387 & 0.01386 \\
\hline $1 \mathrm{M}$ & 0.0145 & 0.01383 & 0.01374 & 0.01373 & 0.01373 \\
\hline $2 \mathrm{M}$ & 0.01440 & 0.01371 & 0.01368 & 0.01368 & 0.01368 \\
\hline
\end{tabular}

Table 8: Average Miss Ratios for Berkeley Multimedia Workload (16 byte Block Size) 
Berkeley Multimedia Workload

\begin{tabular}{|c|c|c|c|c|c|}
\hline & & & & & \\
\hline & & Instr & euction Ce & ache & \\
\hline$S j 7 \mathrm{~S}$ S & & $\frac{\text { Ass }}{\text { vay }}$ & Sociativi & $\frac{t y}{8-w a y}$ & \\
\hline $\begin{array}{l}1 \mathrm{~K} \\
2 \mathrm{~K}\end{array}$ & $\begin{array}{l}0.03410 \\
0.01865\end{array}$ & $\begin{array}{l}0.03361 \\
0.01720\end{array}$ & $\begin{array}{l}0.03194 \\
0.01594\end{array}$ & $\begin{array}{l}0.03103 \\
0.01600\end{array}$ & \\
\hline $4 \mathrm{~K}$ & & & & & \\
\hline $3 \mathrm{~K}$ & & 0.00575 & 0.00433 & 0.00410 & \\
\hline & & & & & \\
\hline & & & & & \\
\hline & & & & & \\
\hline & & & & & \\
\hline & & & & & \\
\hline & & & .0 & $0.0 \quad-1$ & \\
\hline & & & & & \\
\hline & & & & & \\
\hline & & 6 & & & \\
\hline & & & . & & \\
\hline & & & & & \\
\hline & & & & & \\
\hline & & & & & \\
\hline & & & & & \\
\hline & & & & & \\
\hline & & & & & \\
\hline & & 0.00 & & & 0.0 \\
\hline & & & $0.0+c$ & 0.0 & \\
\hline & & & & & \\
\hline & & & & & \\
\hline & & & & & \\
\hline & & & & & \\
\hline & & & & & \\
\hline & & & 0.0 & 0.0 & \\
\hline & & & & & \\
\hline & & & & & \\
\hline 14 & & & & & \\
\hline & & & & & \\
\hline
\end{tabular}

\begin{tabular}{|c|c|c|c|c|c|}
\hline & & & Document & & \\
\hline & & Instit & uction & & \\
\hline$\overline{z e}$ & irect & & 4-way & & 16-way \\
\hline & 0.05230 & 0.05437 & \begin{tabular}{|l|}
0.05363 \\
\end{tabular} & 0.05234 & 0.05268 \\
\hline & $\begin{array}{l}0.03529 \\
0.02202\end{array}$ & $\begin{array}{l}0.03171 \\
0.01694\end{array}$ & $\left|\begin{array}{l}0.02936 \\
0.01310\end{array}\right|$ & $\begin{array}{l}0.02934 \\
0.01041\end{array}$ & $\begin{array}{l}0.02980 \\
0.00822\end{array}$ \\
\hline & 0.01392 & 0.00963 & 0.00454 & 0.00404 & 0.00055 \\
\hline & 0.00535 & 0.00285 & 0.00133 & 0.00032 & 0.00031 \\
\hline & 0.00381 & 0.00027 & $\left|\begin{array}{l}0.00023 \\
0\end{array}\right|$ & 0.00020 & 0.00020 \\
\hline $8 \mathrm{KK}$ & 0.00154 & 0.00011 & $\left|\begin{array}{l}0.00014 \\
0.00011\end{array}\right|$ & 0.00010 & 0.00013 \\
\hline $256 \mathrm{~K}$ & 0.00141 & 0.00010 & 0.00010 & 0.00010 & 0.00010 \\
\hline & 0.00010 & 0.00010 & 0.00010 & 0.00010 & .00010 \\
\hline & & & 0.00010 & 0.00010 & 0.000 \\
\hline & .00010 & 0.00010 & $\frac{0.00010}{+30 c^{2}}$ & 0.00010 & 0.00010 \\
\hline$z e$ & Direct & 2-way & 4 -way & 8-way & 16-way \\
\hline & 0.15503 & 0.11245 & \begin{tabular}{|l|}
0.09384 \\
\end{tabular} & 0.08770 & 0.08201 \\
\hline & 0.06302 & 0.04526 & 0.03124 & 0.02959 & $\begin{array}{l}0.03546 \\
0.02906\end{array}$ \\
\hline & 0.04174 & 0.02793 & 0.02672 & 0.02524 & 0.02495 \\
\hline 16 & 0.03057 & 0.02228 & 0.02173 & 0.02171 & 0.02144 \\
\hline & $\begin{array}{l}0.02600 \\
0.02393\end{array}$ & $\left.\begin{array}{l}0.02042 \\
0.01921\end{array}\right]$ & 0.01979 & 0.01968 & 0.01967 \\
\hline $28 \mathrm{~K}$ & 0.01928 & 0.01886 & 0.01876 & $\begin{array}{l}.01891 \\
0.01875\end{array}$ & 0.01876 \\
\hline $256 \mathrm{~K}$ & 0.01881 & 0.01859 & 0.01858 & 0.01859 & 0.01860 \\
\hline $512 \mathrm{~K}$ & 0.01385 & 0.01718 & 0.01853 & 0.01854 & 0.01852 \\
\hline $1 \mathrm{M}$ & 0.00678 & 0.00670 & 0.00669 & 0.00669 & 0.00669 \\
\hline & 0.0 & & & .00666 & 0.00666 \\
\hline & & & & & \\
\hline & 83 & 34 & \begin{tabular}{|c|}
$4-w a y$ \\
0.08842 \\
\end{tabular} & 750 & $\frac{d y}{785}$ \\
\hline & $\begin{array}{l}.07910 \\
0.05037\end{array}$ & $\begin{array}{l}0.06591 \\
0.03890\end{array}$ & $\left|\begin{array}{l}0.059773 \\
0.03004\end{array}\right|$ & $\begin{array}{l}0.05945 \\
0.02793\end{array}$ & $\begin{array}{l}0.05850 \\
0.02974\end{array}$ \\
\hline & 032 & & 0.01307 & & \\
\hline & & 0.009 & 0.00691 & 0.00522 & \\
\hline $2 \mathrm{~K}$ & 0119 & 0.0 & $\begin{array}{l}0.00473 \\
0.00416\end{array}$ & .00 & 0.00 \\
\hline & & & 0.00400 & 0.0 & 0.0 \\
\hline $256 \mathrm{~K}$ & 05 & 0.0 & $\begin{array}{l}0.00393 \\
0.00391\end{array}$ & $\begin{array}{l}0.00 \\
0.00\end{array}$ & $\begin{array}{l}0.0 \\
0.0\end{array}$ \\
\hline & 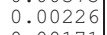 & & 0.001 & 145 & \\
\hline & & & & & \\
\hline
\end{tabular}

Audio
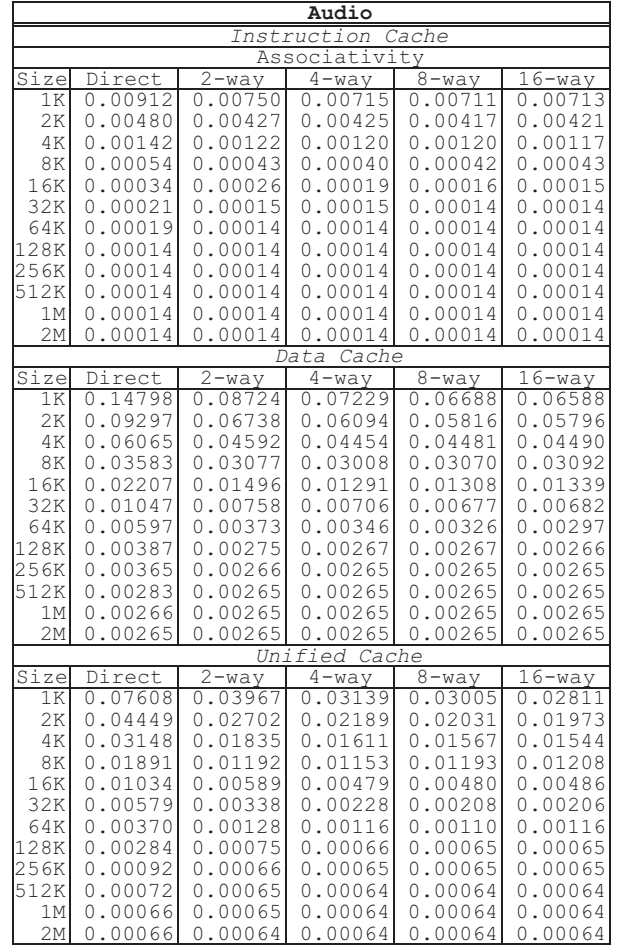

Video

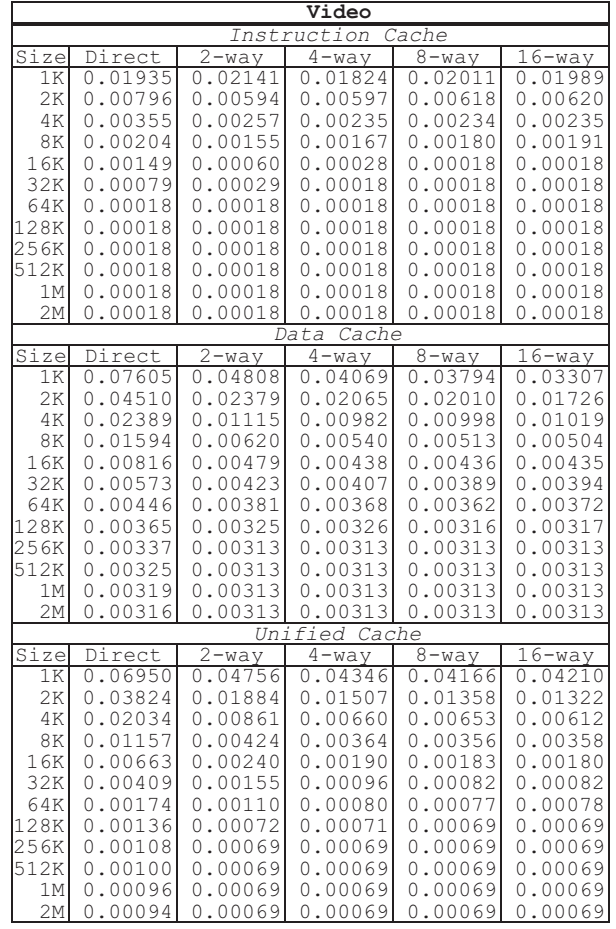

Block Size: 32 bytes Speech

\begin{tabular}{|c|c|c|c|c|c|}
\hline \\
\hline \multirow{2}{*}{\multicolumn{6}{|c|}{$\begin{array}{c}\text { Instruction Cache } \\
\text { Associativity }\end{array}$}} \\
\hline & & & & & \\
\hline$i z e$ & Direct & 2-way & 4-way & 8-way & 16-way \\
\hline $1 \mathrm{~K}$ & 0.04958 & 0.04738 & 0.04289 & 0.03847 & 0.03735 \\
\hline $2 \mathrm{~K}$ & 0.01913 & 0.01830 & 0.01505 & 0.01467 & 0.01469 \\
\hline $4 \mathrm{~K}$ & 0.01372 & 0.01219 & 0.01219 & 0.01224 & 0.01247 \\
\hline $8 \mathrm{~K}$ & 0.00814 & 0.00724 & 0.00596 & 0.00516 & 0.00479 \\
\hline $16 \mathrm{~K}$ & 0.00456 & 0.00321 & 0.00250 & 0.00259 & 0.00258 \\
\hline $32 \mathrm{~K}$ & 0.00235 & 0.00169 & 0.00147 & 0.00125 & 0.00118 \\
\hline $64 \mathrm{~K}$ & 0.00179 & 0.00122 & 0.00115 & 0.00112 & 0.00112 \\
\hline $128 \mathrm{~K}$ & 0.00129 & 0.00116 & 0.00112 & 0.00112 & 0.00112 \\
\hline $256 \mathrm{~K}$ & 0.00121 & 0.00112 & 0.00112 & 0.00112 & 0.00112 \\
\hline $512 \mathrm{~K}$ & 0.00112 & 0.00112 & 0.00112 & 0.00112 & 0.00112 \\
\hline $1 \mathrm{M}$ & 0.00112 & 0.00112 & 0.00112 & 0.00112 & 0.00112 \\
\hline $2 \mathrm{M}$ & 0.00112 & 0.00112 & 0.00112 & 0.00112 & 0.00112 \\
\hline \multicolumn{6}{|c|}{ Data Cache } \\
\hline$z e$ & Direct & 2-way & 4-way & 8-way & -way \\
\hline $1 \mathrm{~K}$ & 0.07420 & 0.04001 & 0.03593 & 0.03096 & 0.02884 \\
\hline $2 \mathrm{~K}$ & 0.04213 & 0.02068 & 0.01655 & 0.01581 & 0.01493 \\
\hline $4 \mathrm{~K}$ & 0.02118 & 0.01293 & 0.01085 & 0.00981 & 0.00985 \\
\hline $8 \mathrm{~K}$ & 0.01461 & 0.00941 & 0.00778 & 0.00766 & 0.00761 \\
\hline $16 \mathrm{~K}$ & 0.00986 & 0.0 & 0.00727 & 0.00740 & 0.00742 \\
\hline $32 \mathrm{~K}$ & 0.00616 & 0.00497 & 0.00484 & 0.00448 & 0.00417 \\
\hline $64 \mathrm{~K}$ & 0.00441 & 0.0 & 0.00267 & 0.00249 & 0.00243 \\
\hline $128 \mathrm{~K}$ & 0.00313 & 0.0 & 0.00243 & 0.00238 & 0.00238 \\
\hline $256 \mathrm{~K}$ & 0.00250 & 0.00239 & 0.00238 & 0.00238 & 0.00238 \\
\hline $12 \mathrm{~K}$ & 0.00239 & 0.0 & 0.00238 & 0.0 & 238 \\
\hline $1 \mathrm{M}$ & 0.00238 & 0.00238 & 0.00238 & 0.00238 & 0.00238 \\
\hline $2 \mathrm{M}$ & 0.00238 & 0.00238 & 0.00238 & 0.00238 & 0.00238 \\
\hline \multicolumn{6}{|c|}{ fied $\mathrm{CaC}$} \\
\hline ize & Dir & & 4-way & $8-$ & \\
\hline $1 \mathrm{~K}$ & 0.10475 & 0.07210 & 0.07099 & 0.06889 & 0.06934 \\
\hline $2 \mathrm{~K}$ & 0.06915 & 0.04156 & 0.04628 & 0.04264 & 0.03992 \\
\hline $4 \mathrm{~K}$ & 0.04254 & 0.01961 & 0.01648 & 0.01584 & 0.01578 \\
\hline $8 \mathrm{~K}$ & 0.02599 & 0.01174 & 0.01033 & 0.01025 & 0.01017 \\
\hline $16 \mathrm{~K}$ & 0.01979 & 0.00643 & 0.00492 & 0.00417 & 0.00397 \\
\hline $32 \mathrm{~K}$ & 0.01603 & 0.00412 & 0.00365 & 0.00363 & 0.00366 \\
\hline $64 \mathrm{~K}$ & 0.01372 & 0.00248 & 0.00214 & 0.00212 & 0.00186 \\
\hline $128 \mathrm{~K}$ & 0.00273 & 0.00179 & 0.00150 & 0.00139 & 0.00138 \\
\hline $256 \mathrm{~K}$ & 0.00245 & 0.00141 & 0.00139 & 0.00138 & 0.00138 \\
\hline $512 \mathrm{~K}$ & 0.00165 & 0.00138 & 0.00138 & 0.00138 & 0.00138 \\
\hline $1 \mathrm{M}$ & 0.00164 & 0.00138 & 0.00138 & 0.00138 & 0.00138 \\
\hline $2 \mathrm{M}$ & 0.00164 & 0.00138 & 0.00138 & 0.00138 & 0.00138 \\
\hline
\end{tabular}

3D

\begin{tabular}{|c|c|c|c|c|c|}
\hline & & & & & \\
\hline & & Instr & tion $C$ & ache & \\
\hline$i z$ & ires & 2-way & 4-way & 8-way & 16-way \\
\hline $1 \mathrm{~K}$ & 0.04016 & 0.03739 & 0.03780 & 0.03712 & 0.03751 \\
\hline $2 \mathrm{~K}$ & 0.02606 & 0.02578 & 0.02507 & 0.02561 & 0.02601 \\
\hline $4 \mathrm{~K}$ & 0.01703 & 0.01498 & 0.01208 & 0.01138 & 0.01098 \\
\hline $8 \mathrm{~K}$ & 0.01126 & 0.00990 & 0.00906 & 0.00905 & 0.00922 \\
\hline $16 \mathrm{~K}$ & 0.00766 & 0.00556 & 0.00530 & 0.00521 & 0.00547 \\
\hline $32 \mathrm{~K}$ & 0.00430 & 0.00218 & 0.00154 & 0.00091 & 0.00039 \\
\hline $64 K$ & 0.00251 & 0.00082 & 0.00042 & 0.00032 & 0.00030 \\
\hline $128 \mathrm{~K}$ & 0.00181 & 0.00030 & 0.00029 & 0.00028 & 0.00028 \\
\hline $256 \mathrm{~K}$ & 0.00106 & 0.00029 & 0.00028 & 0.00028 & 0.00028 \\
\hline $512 \mathrm{~K}$ & 0.00068 & 0.00028 & 0.00028 & 0.00028 & 0.00028 \\
\hline $1 \mathrm{M}$ & 0.0 & 0.00028 & 0.00028 & 0.00028 & 0.00028 \\
\hline $2 \mathrm{M}$ & 0.0 & 0.0 & 0.00028 & 0.00028 & 0.00028 \\
\hline & & & ata $\mathrm{Ca}$ & & \\
\hline $\mathrm{ze}$ & D. & $2-$ & 4-way & 8-way & way \\
\hline $1 \mathrm{~K}$ & 0.14628 & 0.11505 & 0.10865 & 0.10616 & 0.10651 \\
\hline $2 \mathrm{~K}$ & 0.1 & 0.09295 & 0.08709 & 0.08494 & 0.08356 \\
\hline $4 \mathrm{~K}$ & 0.08853 & 0.07735 & 0.07401 & 0.07355 & 0.07355 \\
\hline $8 \mathrm{~K}$ & 0.06933 & 0.06159 & 0.06007 & 0.05943 & 0.05905 \\
\hline $16 \mathrm{~K}$ & 0.05733 & 0.04986 & 0.04850 & 0.04811 & 0.04819 \\
\hline $32 \mathrm{~K}$ & 0.04743 & 0.04139 & 0.03969 & 0.03878 & 0.03806 \\
\hline $64 \mathrm{~K}$ & 0.0 & 0.0 & 0.03475 & 0.03432 & 0.03413 \\
\hline $128 \mathrm{~K}$ & 0.03505 & 0.03367 & 0.03285 & 0.03280 & 0.03282 \\
\hline $256 \mathrm{~K}$ & 0.03321 & 0.0 & 0.03142 & 0.03141 & 0.03141 \\
\hline 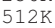 & 0.0 & 0.0 & 0.0 & 0.0 & 0.03051 \\
\hline $1 \mathrm{M}$ & 0.0 & 0.0 & 0.03 & 0.03 & 0.03014 \\
\hline $2 \mathrm{M}$ & 0. & 0.0 & 0.03002 & 0.03001 & 0.03001 \\
\hline & & & ied & & \\
\hline ize & Dir & $2-w a y$ & 4-wa & 8-way & 16-way \\
\hline $1 \mathrm{~K}$ & 0.10513 & 0.07677 & 0.07115 & 0.07008 & 0.06982 \\
\hline $2 \mathrm{~K}$ & 0.07982 & 0.05779 & 0.05483 & 0.05434 & 0.05377 \\
\hline $4 \mathrm{~K}$ & 0.0 & 0.04138 & 0.03823 & 0.03678 & 0.03686 \\
\hline $8 \mathrm{~K}$ & 0.03558 & 0.02875 & 0.02667 & 0.02552 & 0.02545 \\
\hline $16 \mathrm{~K}$ & 0.02738 & 0.02137 & 0.02043 & 0.01999 & 0.02001 \\
\hline $32 \mathrm{~K}$ & 0.02012 & 0.01634 & 0.01535 & 0.01527 & 0.01549 \\
\hline $64 \mathrm{~K}$ & 0.01537 & 0.01183 & 0.01016 & 0.00946 & 0.00906 \\
\hline $128 \mathrm{~K}$ & 0.01179 & 0.00907 & 0.00808 & 0.00782 & 0.00778 \\
\hline & 0.0102 & 0.00 & 0.00747 & 0.00746 & 0.00747 \\
\hline $512 \mathrm{~K}$ & 0.00854 & 0.00741 & 0.00717 & 0.00714 & 0.00712 \\
\hline $1 \mathrm{M}$ & 0.00771 & 0.00712 & 0.00704 & 0.00703 & 0.00704 \\
\hline $2 \mathrm{M}$ & 0.00760 & 0.00703 & 0.00701 & 0.00700 & 0.00700 \\
\hline
\end{tabular}

Table 9: Average Miss Ratios for Berkeley Multimedia Workload (32 byte Block Size) 
Berkeley Multimedia Workload

\begin{tabular}{|c|c|c|c|c|c|}
\hline & & & ultime & & \\
\hline & & Instr & cuct. & ache & \\
\hline & & & $c i a t i$ & & \\
\hline $\mathrm{ze}$ & & & & $8-$ & 16-way \\
\hline $\begin{array}{l}1 \mathrm{~K} \\
2 \mathrm{~K}\end{array}$ & $\begin{array}{l}0.0 \\
0.0\end{array}$ & $\begin{array}{l}02324 \\
01212\end{array}$ & $\begin{array}{l}162 \\
162\end{array}$ & $\begin{array}{l}0 \\
0 \\
0\end{array}$ & \\
\hline & 0.0 & 0.00699 & 0.0 & 0.00 & \\
\hline & & & & & \\
\hline & & & 0.0 & & \\
\hline & & & & & \\
\hline k & 0.08 & 0.00 & 0.0 & 0.0 & 0 . \\
\hline & & & & & \\
\hline & & & & & \\
\hline & & & & & \\
\hline & & 021 & 0.00021 & 0.00021 & 0.00021 \\
\hline & & & & & \\
\hline & & & & & \\
\hline 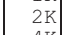 & 0. & & 0.0 & & \\
\hline 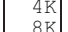 & 0. & 0. & 0.02 & 0.0 & \\
\hline & & & & & \\
\hline k & & & & & \\
\hline & & & & & \\
\hline & & & & & \\
\hline & & & & & \\
\hline 1 & & & 0.9 & & \\
\hline & & & & & \\
\hline & & & & & \\
\hline & & & & $\frac{8-1}{0.0}$ & $16-\bar{n}$ \\
\hline & & & & & \\
\hline & & & 0.0 & & \\
\hline & & & & & \\
\hline & & & & & \\
\hline & & & & & \\
\hline & & & & & \\
\hline & & & & & \\
\hline & & & 0.00 & 0.0 & 0.0 \\
\hline & & & & & \\
\hline
\end{tabular}

\begin{tabular}{|c|c|c|c|c|c|}
\hline & & & Document & & \\
\hline & & Inst & & ache & \\
\hline ize & Direct & & 4-way & 8-way & 16-way \\
\hline $\begin{array}{c}1 \mathrm{~K} \\
2 \mathrm{~K}\end{array}$ & $\begin{array}{l}0.03697 \\
0.02702\end{array}$ & $\begin{array}{l}0.03832 \\
0.02462\end{array}$ & $\left|\begin{array}{l}0.03783 \\
0.02392\end{array}\right|$ & $\left|\begin{array}{l}0.03684 \\
0.02357\end{array}\right|$ & \\
\hline & 0.01741 & 0.01402 & 0.01149 & 0.01109 & 0.01252 \\
\hline & 0.01099 & 0.00754 & 0.00377 & 0.00408 & 0.00041 \\
\hline $\begin{array}{l}16 \mathrm{~K} \\
32 \mathrm{~K}\end{array}$ & $\begin{array}{l}0.00444 \\
0.00288\end{array}$ & $\begin{array}{l}0.00196 \\
0.00019\end{array}$ & $\left.\mid \begin{array}{l}0.00123 \\
0.0016\end{array}\right]$ & $\left|\begin{array}{l}0.00023 \\
0.00013\end{array}\right|$ & $\begin{array}{l}0.00021 \\
0.00012\end{array}$ \\
\hline & 0.00278 & 0.00011 & 0.00009 & 0.00009 & 0.00009 \\
\hline $128 \mathrm{~K}$ & 0.00146 & 0.00007 & 0.00006 & 0.00006 & 0.00006 \\
\hline $\begin{array}{l}256 \mathrm{~K} \\
512 \mathrm{~K}\end{array}$ & $\begin{array}{l}0.00137 \\
0.00006\end{array}$ & $\begin{array}{l}0.00006 \\
0.00006\end{array}$ & {$\left[\begin{array}{l}0.00006 \\
0.0006\end{array}\right]$} & $\begin{array}{l}0.00006 \\
0.00006\end{array}$ & $\begin{array}{l}0.00006 \\
0.00006\end{array}$ \\
\hline $1 \mathrm{M}$ & 0.00006 & 0.00006 & 0.00006 & 0.00006 & 0.00006 \\
\hline & 0.00006 & 0.00006 & 0.00006 & 0.00006 & 0.00006 \\
\hline & & & & & 16-way \\
\hline & & & & & $16-\mathrm{way}$ \\
\hline & .11173 & 0.07683 & 0.05868 & 0.05008 & 0.04472 \\
\hline & $\begin{array}{l}0.06610 \\
0.04343\end{array}$ & $\begin{array}{l}0.04835 \\
0.0264\end{array}$ & {$\left[\begin{array}{l}0.03039 \\
0.02249\end{array}\right]$} & 0.02468 & 0.02375 \\
\hline & $\begin{array}{l}0.04343 \\
0.02949\end{array}$ & $\begin{array}{l}0.02644 \\
0.01840\end{array}$ & {$\left[\begin{array}{l}0.02249 \\
0.01775\end{array}\right.$} & 0.02056 & 0.02020 \\
\hline & 0.02 & 0.0 & 0.01640 & 0.01634 & 0.01635 \\
\hline & 0.02 & 0. & 0.01581 & 0.01578 & 0.01577 \\
\hline & 0.01628 & 0.01574 & 0.01568 & 0.01567 & 0.01568 \\
\hline $6 \mathrm{~K}$ & 001577 & 0. & 0. & 0.01556 & 0.01 \\
\hline $2 \mathrm{~K}$ & 0000368 & 0. & & 0.0 & 0.01 \\
\hline t & .0030 & .0001 & 0.003 & 0.00357 & 0.00357 \\
\hline & & & & & \\
\hline & & & & & $16-$ \\
\hline & 077 & 0.05765 & 0.0508 & 0.04910 & 0.04 \\
\hline & & 0.03 & 0.02 & & \\
\hline & $\begin{array}{l}.03259 \\
01680\end{array}$ & $\begin{array}{l}0.01895 \\
0.0881\end{array}$ & $\begin{array}{l}0.01378 \\
0.00629\end{array}$ & $\begin{array}{l}0.01259 \\
0.00424\end{array}$ & $\begin{array}{l}0.00813 \\
0.00416\end{array}$ \\
\hline & . & & 0.0 & & \\
\hline 64. & 0.00921 & 0.0 & 0.00345 & 0.0 & 0.00 \\
\hline $86 \mathrm{~K}$ & & $\begin{array}{l}0.0 \\
0.0\end{array}$ & & $\left|\begin{array}{l}0.00332 \\
0.00328\end{array}\right|$ & \\
\hline & .00 & & 0.00326 & 26 & \\
\hline & .00151 & $\begin{array}{l}0.00079 \\
0.00078\end{array}$ & 0.00079 & 0.00078 & $\begin{array}{l}0.00079 \\
0.00078\end{array}$ \\
\hline & & & & & \\
\hline
\end{tabular}

Audio
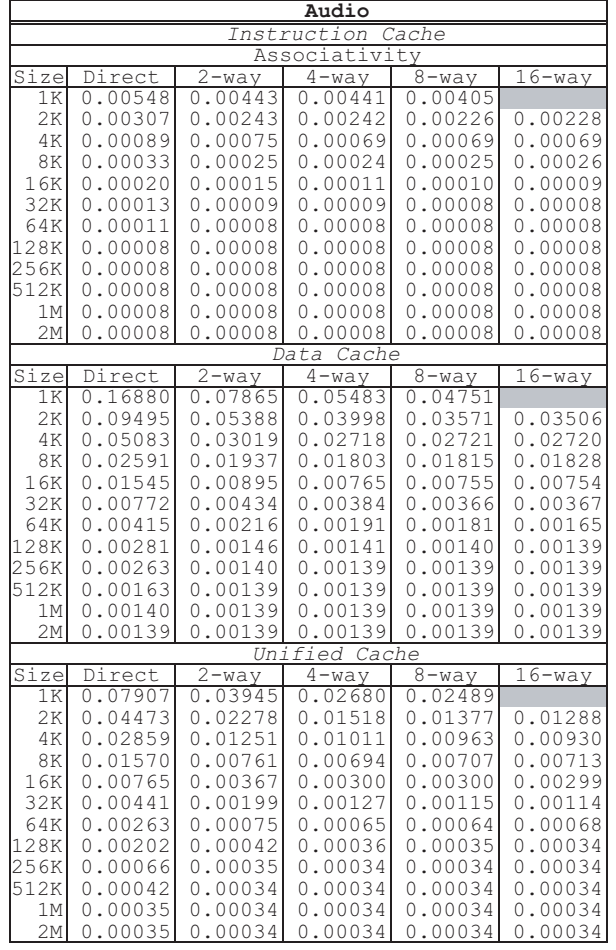

Video
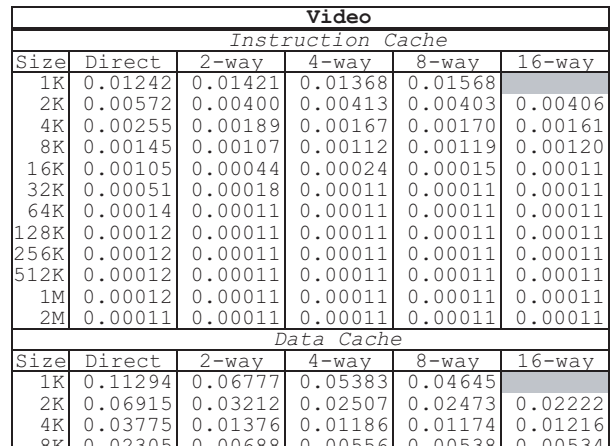

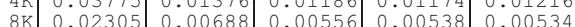

\begin{tabular}{l|l|l|l|l|l|}
$16 \mathrm{~K}$ & 0.00906 & 0.00425 & 0.00335 & 0.00314 & 0.00310
\end{tabular}

\begin{tabular}{|l|l|l|l|l|l|}
$32 \mathrm{~K}$ & 0.00557 & 0.00317 & 0.00291 & 0.00296 & 0.00296 \\
$64 \mathrm{~K}$ & 0.00393 & 0.00288 & 0.00273 & 0.00252 & 0.00250
\end{tabular}

\begin{tabular}{l|l|l|l|l|l|l|}
$128 \mathrm{~K}$ & 0.00275 & 0.00208 & 0.00208 & 0.00190 & 0.00191
\end{tabular}

\begin{tabular}{|l|l|l|l|l|l|}
$256 \mathrm{~K}$ & 0.00222 & 0.00180 & 0.00177 & 0.00177 & 0.00176 \\
$512 \mathrm{~K}$ & 0.00199 & 0.00176 & 0.00176 & 0.00176 & 0.00176 \\
\hline
\end{tabular}

\begin{tabular}{l|l|l|l|l|l|l|}
$1 \mathrm{M}$ & 0.00186 & 0.00176 & 0.00176 & 0.00176 & 0.00176 \\
$2 \mathrm{M}$ & 0.00180 & 0.00176 & 0.00176 & 0.00176 & 0.00176 \\
\hline
\end{tabular}

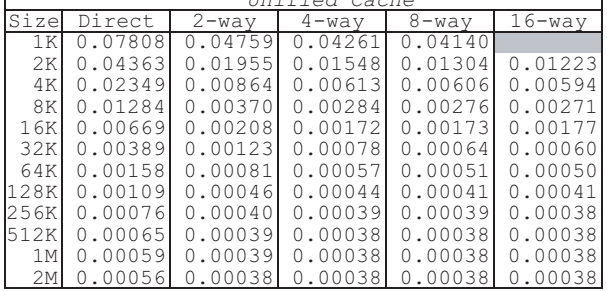

Block Size: 64 bytes Speech

\begin{tabular}{|c|c|c|c|c|c|}
\hline \\
\hline \multirow{2}{*}{\multicolumn{6}{|c|}{$\begin{array}{c}\text { Instruction Cache } \\
\text { Associativity }\end{array}$}} \\
\hline & & & & & \\
\hline $\mathrm{ize}$ & Direct & 2-way & 4-way & 8-way & 16-way \\
\hline $1 \mathrm{~K}$ & 0.03435 & 0.03221 & 0.02519 & 0.02781 & \\
\hline $2 \mathrm{~K}$ & 0.01217 & 0.01093 & 0.00928 & 0.00947 & 0.00904 \\
\hline $4 \mathrm{~K}$ & 0.00862 & 0.00766 & 0.00761 & 0.00742 & 0.00746 \\
\hline $8 \mathrm{~K}$ & 0.00531 & 0.00472 & 0.00440 & 0.00441 & 0.00435 \\
\hline $16 \mathrm{~K}$ & 0.00295 & 0.00207 & 0.00183 & 0.00146 & 0.00145 \\
\hline $32 \mathrm{~K}$ & 0.00157 & 0.00104 & 0.00085 & 0.00078 & 0.00073 \\
\hline $64 \mathrm{~K}$ & 0.00108 & 0.00067 & 0.00062 & 0.00060 & 0.00060 \\
\hline $128 \mathrm{~K}$ & 0.00070 & 0.00063 & 0.00060 & 0.00060 & 0.00060 \\
\hline $256 \mathrm{~K}$ & 0.00065 & 0.00060 & 0.00060 & 0.00060 & 0.00060 \\
\hline $512 \mathrm{~K}$ & 0.00060 & 0.00060 & 0.00060 & 0.00060 & 0.00060 \\
\hline $1 \mathrm{M}$ & 0.00060 & 0.00060 & 0.00060 & 0.00060 & 0.00060 \\
\hline $2 \mathrm{M}$ & 0.00060 & 0.00060 & 0.00060 & 0.00060 & 0.00060 \\
\hline \multicolumn{6}{|c|}{ Data Cache } \\
\hline$z e$ & Direct & $2-w a y$ & 4-way & $8-w a y$ & $16-w a y$ \\
\hline $1 \mathrm{~K}$ & 0.09726 & 0.06199 & 0.05679 & 0.05744 & \\
\hline $2 \mathrm{~K}$ & 0.06272 & 0.03005 & 0.01789 & 0.01546 & 0.01512 \\
\hline $4 \mathrm{~K}$ & 0.03631 & 0.01226 & 0.00935 & 0.00802 & 0.00752 \\
\hline $8 \mathrm{~K}$ & 0.01513 & 0.00730 & 0.00532 & 0.00514 & 0.00501 \\
\hline $16 \mathrm{~K}$ & 0.00800 & 0.00501 & 0.00467 & 0.00465 & 0.00465 \\
\hline $32 \mathrm{~K}$ & 0.00454 & 0.00353 & 0.00355 & 0.00374 & 0.00392 \\
\hline $64 \mathrm{~K}$ & 0.00312 & 0.0 & 0.00178 & 0.00162 & 0147 \\
\hline $128 \mathrm{~K}$ & 0.00207 & 0.00168 & 0.00146 & 0.00137 & 0.00137 \\
\hline $256 \mathrm{~K}$ & 0.00149 & 0.00138 & 0.00137 & 0.00137 & 0.00137 \\
\hline $12 \mathrm{~K}$ & 0.00138 & 0.00137 & 0.00137 & 0.00137 & 0137 \\
\hline $1 \mathrm{M}$ & 0.00137 & 0.00137 & 0.00137 & 0.00137 & 0.00137 \\
\hline $2 \mathrm{M}$ & 0.00137 & 0.00137 & 0.00137 & 0.00137 & 0.00137 \\
\hline \multicolumn{6}{|c|}{ fied cache } \\
\hline $\mathrm{ze}$ & & $2-$ & 4-way & 8-wa & 16-way \\
\hline $1 \mathrm{~K}$ & 0.08 & 0.06051 & 0.05602 & 0.04859 & \\
\hline $2 \mathrm{~K}$ & 0.06053 & 0.03807 & 0.03618 & 0.03470 & 0.03823 \\
\hline $4 \mathrm{~K}$ & 0.03746 & 0.01752 & 0.01321 & 0.01127 & 0.01085 \\
\hline $8 \mathrm{~K}$ & 0.01918 & 0.00874 & 0.00765 & 0.00777 & 0.00824 \\
\hline $16 \mathrm{~K}$ & 0.01295 & 0.00451 & 0.00368 & 0.00282 & 0.00263 \\
\hline $32 \mathrm{~K}$ & 0.00998 & 0.00276 & 0.00230 & 0.00219 & 0.00218 \\
\hline $64 \mathrm{~K}$ & 0.00810 & 0.00160 & 0.00149 & 0.00150 & 0.00151 \\
\hline $128 \mathrm{~K}$ & 0.00197 & 0.00108 & 0.00090 & 0.00076 & 0.00076 \\
\hline $256 \mathrm{~K}$ & 0.00167 & 0.00079 & 0.00076 & 0.00075 & 0.00075 \\
\hline $512 \mathrm{~K}$ & 0.00099 & 0.00075 & 0.00075 & 0.00075 & 0.00075 \\
\hline $1 \mathrm{M}$ & 0.00099 & 0.00075 & 0.00075 & 0.00075 & 0.00075 \\
\hline $2 \mathrm{M}$ & 0.00099 & 0.00075 & 0.00075 & 0.00075 & 0.00075 \\
\hline
\end{tabular}

3D

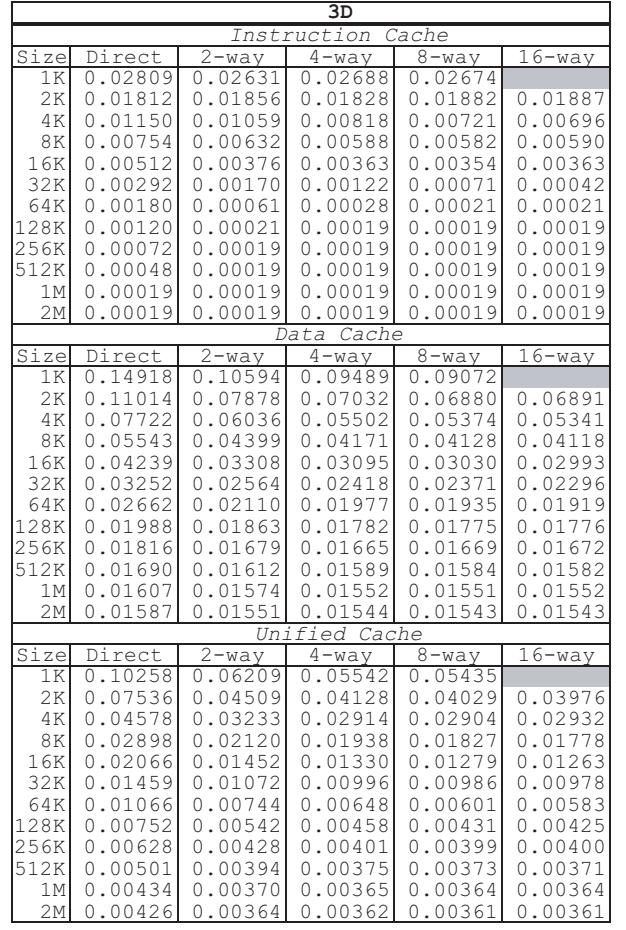

Table 10: Average Miss Ratios for Berkeley Multimedia Workload (64 byte Block Size) 
Berkeley Multimedia Workload

\begin{tabular}{|c|c|c|c|c|c|}
\hline & & & ultime & & \\
\hline & & Inst. & cuct. & ache & \\
\hline & & Ass & sociati & & \\
\hline Th & Dir & & & 8-way & 16-way \\
\hline $\begin{array}{l}1 \mathrm{~K} \\
2 \mathrm{~K}\end{array}$ & $\begin{array}{l}0.01 \\
0.00\end{array}$ & $\begin{array}{l}0.01599 \\
0.00828\end{array}$ & $\begin{array}{l}0.01564 \\
0.00831\end{array} \mid$ & & \\
\hline & 0.0 & 0.00 & 0.0 & & 0.00429 \\
\hline & 0.08 & & 0.0 & & \\
\hline & & & & & \\
\hline & & & & & \\
\hline & & & & 0.0 & \\
\hline & & & & & \\
\hline & & & & 0.0 & \\
\hline & & & .0 & & \\
\hline & & & 00 & 0.00011 & 0.00011 \\
\hline & & & & $8-1$ & \\
\hline & & & & & \\
\hline $2 \mathrm{~K}$ & 0. & & & 15 & \\
\hline & & $\begin{array}{l}96 \\
95\end{array}$ & 0.02 & & \\
\hline & & 5 & & 0.0 & \\
\hline & & & & & \\
\hline & & & & & \\
\hline & & 00 & & 0.0 & \\
\hline & & 0.0 & 0.0 & 0.00 & \\
\hline $\begin{array}{r}12 \mathrm{~K} \\
1 \mathrm{M}\end{array}$ & & 0.0 & & & \\
\hline & & & & & \\
\hline & & & & & \\
\hline & & & & & \\
\hline & & & & & \\
\hline & 0. & & 0.0 & 0.8 & \\
\hline & & & & & \\
\hline & & & & & \\
\hline & & & & & \\
\hline 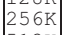 & & & & & \\
\hline & & & & & \\
\hline & & & 0.000 & 0.00 & 0.000 \\
\hline & & & & & \\
\hline
\end{tabular}

\begin{tabular}{|c|c|c|c|c|c|}
\hline & & & Document & & \\
\hline & & Inst & cuction C & ac & \\
\hline ize & Dir & & & 8-way & 16-way \\
\hline $2 \mathrm{~K}$ & 0.02119 & 0.01910 & 0.02022 & 0.02057 & \\
\hline & 0.01363 & 0.01139 & 0.01090 & 0.01003 & 0.01044 \\
\hline $\begin{array}{ll}8 \mathrm{~K} \\
6 \mathrm{~K}\end{array}$ & 0.00910 & $\begin{array}{l}0.00630 \\
0.00200\end{array}$ & $\left|\begin{array}{l}0.00460 \\
0.0018\end{array}\right|$ & $\begin{array}{l}0.00450 \\
0.00018\end{array}$ & $\begin{array}{l}0.00091 \\
0.00017\end{array}$ \\
\hline & 0.00255 & 0.00014 & 0.00012 & 0.00009 & 0.00009 \\
\hline $64 \mathrm{~K}$ & 0.00248 & 0.00008 & 0.00006 & 0.00006 & 0.00006 \\
\hline $\begin{array}{l}128 \mathrm{~K} \\
256 \mathrm{~K}\end{array}$ & 0.00091 & 0.00004 & $\begin{array}{l}0.00004 \\
0.00004\end{array}$ & 0.00004 & $\begin{array}{l}0.00004 \\
0.00004\end{array}$ \\
\hline $512 \mathrm{~K}$ & 0.00004 & 0.00004 & 0.00004 & 0.00004 & 0.00004 \\
\hline & 0.00004 & 0.00004 & 0.00004 & 0.00004 & 0.000 \\
\hline & 0.00004 & 0.00004 & 0.00004 & 0.00004 & 0.00004 \\
\hline & & & $4-$ & 8-way & $16-w a$ \\
\hline $1 \mathrm{n}$ & & & & & \\
\hline & 33 & 635 & 610 & 0.06996 & \\
\hline $8 \mathrm{~K}>2 \mathrm{x}$ & $\begin{array}{l}0.07930 \\
0.05123\end{array}$ & $\begin{array}{l}0.05551 \\
0.02638\end{array}$ & $\left|\begin{array}{l}0.03486 \\
0.02141\end{array}\right|$ & $\begin{array}{l}0.02659 \\
0.01853\end{array}$ & $\begin{array}{l}0.02278 \\
0.01773\end{array}$ \\
\hline $16 \mathrm{~K}$ & 0.03277 & 0.01720 & 0.01587 & 0.01571 & 0.01550 \\
\hline & $0.02-2$ & 0.01544 & 0.01471 & 0.01465 & 0.01 \\
\hline $\begin{array}{r}64 \mathrm{~K} \\
128 \mathrm{~K}\end{array}$ & $\begin{array}{l}0.02170 \\
0.01646\end{array}$ & $\begin{array}{l}0.01449 \\
0.01420\end{array}$ & $\begin{array}{l}0.01424 \\
0.01413\end{array} \mid$ & $\begin{array}{l}0.01421 \\
0.01412\end{array}$ & $\begin{array}{l}0.01421 \\
0.01412\end{array}$ \\
\hline $256 \mathrm{~K}$ & 0.01431 & 0.01403 & 0.01403 & 0.01404 & 0.01404 \\
\hline $512 \mathrm{~K}$ & 0.00 & 0.01 & 0.01400 & 0.01400 & 0.01401 \\
\hline $1 \mathrm{M}$ & 0.00213 & 0.00206 & 0.00205 & 0.00205 & 0.00205 \\
\hline & & & Fied & 0.00 & 2 \\
\hline & & 2 & $4-w$ & 8-way & 16-way \\
\hline & & 0.0 & 0.04690 & 0 . & \\
\hline & & 0.03484 & 0.03 & & \\
\hline & .03 & $\begin{array}{l}0.02221 \\
0.01052\end{array}$ & $\begin{array}{l}0.01509 \\
0.00735\end{array}$ & $\begin{array}{l}0.01390 \\
0.00516\end{array}$ & $\begin{array}{l}0.01312 \\
0.00374\end{array}$ \\
\hline & & & 0.00 & 0.0 & \\
\hline $64 \mathrm{~F}$ & 0.01 & 0.0 & 0.00310 & 0.00307 & 0.0 \\
\hline & 0 & $\begin{array}{l}0.0 \\
0.0\end{array}$ & & $\begin{array}{l}0.00299 \\
0.00295\end{array}$ & \\
\hline & & & 0.00294 & 0.00294 & 0.00 \\
\hline & 0 & 0.0 & 0.00045 & 0.00045 & 0.00045 \\
\hline & & & & & \\
\hline
\end{tabular}
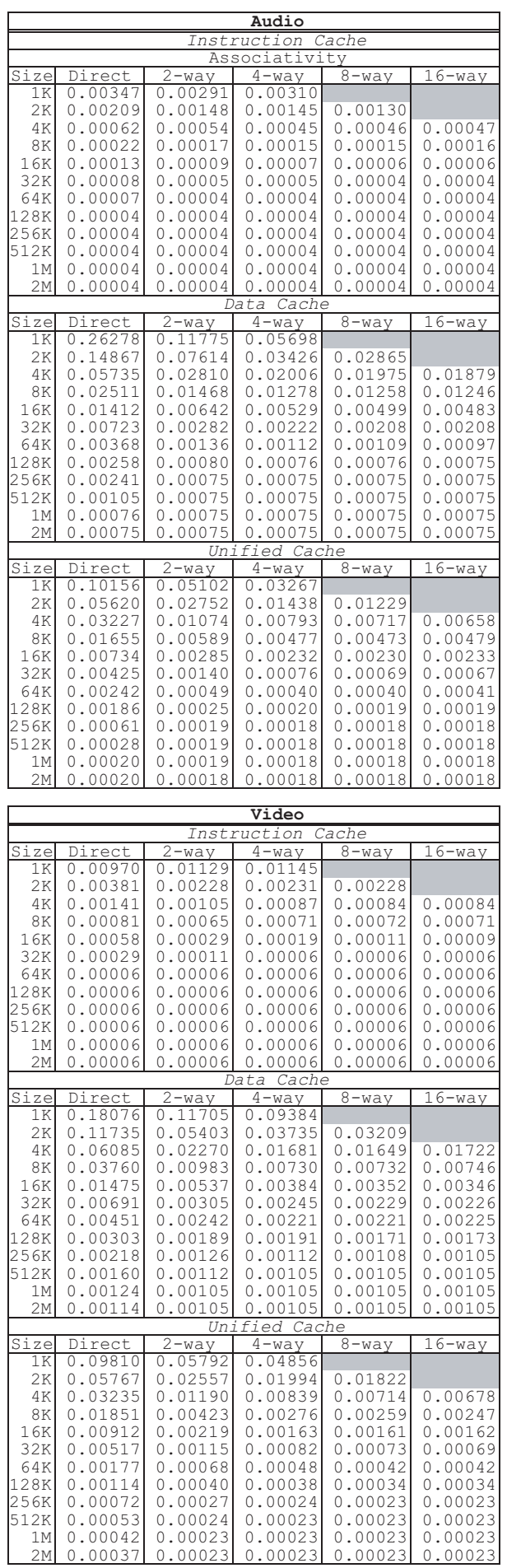

Block Size: 128 bytes Speech

\begin{tabular}{|c|c|c|c|c|c|}
\hline \\
\hline \multirow{2}{*}{\multicolumn{6}{|c|}{$\begin{array}{c}\text { Instruction Cache } \\
\text { Associativity }\end{array}$}} \\
\hline \multicolumn{3}{|c|}{ Associativity } & & & \\
\hline Size & Direct & 2-way & 4-way & 8-way & $16-\mathrm{wc}$ \\
\hline $1 \mathrm{~K}$ & 0.02762 & 0.02146 & 0.01861 & & \\
\hline $2 \mathrm{~K}$ & 0 & & 0.00620 & 0.00648 & \\
\hline $4 \mathrm{~K}$ & 0.0058 & 0.0 & 0.00499 & 0.00490 & 0.004 \\
\hline $8 \mathrm{~K}$ & & & 0.00381 & 0.00409 & \\
\hline $16 \mathrm{~K}$ & 0 & 0. & 0.00121 & 0. & \\
\hline $32 \mathrm{~K}$ & & 0.00069 & 0.00056 & 0.00057 & 0 \\
\hline $64 \mathrm{~K}$ & 0. & & & & \\
\hline $28 \mathrm{~K}$ & & & 0.00033 & & \\
\hline $56 \mathrm{~K}$ & 0.0 & & 0.00033 & 0.00033 & \\
\hline$\pi$ & & & & & \\
\hline $1 \mathrm{M}$ & & 033 & 0.00033 & 0.0 & \\
\hline $2 \mathrm{M}$ & & & 0.0 & & \\
\hline \multicolumn{6}{|c|}{ Data Cache } \\
\hline ize & & 2-way & 4-way & 8-way & 16-way \\
\hline $1 \mathrm{~K}$ & & & 0.06541 & & \\
\hline $2 \mathrm{~K}$ & & & & & \\
\hline $4 \mathrm{~K}$ & & & 0.0 & & \\
\hline $8 \mathrm{~K}$ & 0. & 0.0 & 0.00 & 55 & \\
\hline $16 \mathrm{~K}$ & & & 0. & 0. & \\
\hline $32 \mathrm{~K}$ & & & 0.0 & & \\
\hline $64 \mathrm{~K}$ & & & & & \\
\hline $28 \mathrm{~K}$ & & & 0. & & \\
\hline $56 \mathrm{~K}$ & 0.0 & 0. & 0.0 & 0. & \\
\hline $12 \mathrm{~K}$ & & & 0.0 & & \\
\hline 11 & & & 0.0 r -9 & & \\
\hline 1 & & 0. & 0.00080 & 0.0 & \\
\hline \multicolumn{6}{|c|}{ Unified Cache } \\
\hline ize & & & & $8-v$ & \\
\hline & & & & & \\
\hline $2 \mathrm{~F}$ & & & 0. & & \\
\hline $4 \mathrm{~K}$ & 0. & 0.0 & 0.01243 & 0.0 & \\
\hline 81 & & & & & \\
\hline $16 \mathrm{~K}$ & 0. & 0.0 & 0.0 a 0 & & \\
\hline $32 \mathrm{~K}$ & & & 0.0 & 0.0 & \\
\hline $64 \mathrm{~K}$ & & & 0.0 & & \\
\hline 28 & 0.0017 & & 0.00059 & & \\
\hline & 0.00145 & 0.0 & 0.00044 & 0.00042 & \\
\hline & & & 0.00042 & 0.00 & \\
\hline 1 & & & & & \\
\hline 2 & & 0. & 0.0 & 0.0 & 0. \\
\hline
\end{tabular}

3D

\begin{tabular}{|c|c|c|c|c|c|}
\hline \\
\hline \multicolumn{6}{|c|}{\begin{tabular}{l|l|l}
\multicolumn{3}{l}{ Instruction } \\
way
\end{tabular}} \\
\hline $1 \mathrm{~K}$ & & & 0. & & \\
\hline $2 \mathrm{~K}$ & & & & & \\
\hline $4 \mathrm{~K}$ & & 8 & 0.00554 & & 0.004 \\
\hline $8 \mathrm{~K}$ & & & & & \\
\hline $16 \mathrm{~K}$ & & & & & \\
\hline $32 \mathrm{~K}$ & & & & & \\
\hline $64 \mathrm{~K}$ & & & & & \\
\hline & & & & & \\
\hline 56 & & & 0. & & \\
\hline & & & & & \\
\hline 1 & & & 0. & & \\
\hline 2 & & & & & \\
\hline \multicolumn{6}{|c|}{ Data Cach } \\
\hline $\mathrm{ze}$ & & & & $8-x$ & $16-$ \\
\hline $1 \mathrm{~K}$ & & & & & \\
\hline 21 & & & & & \\
\hline 4 & & & & & \\
\hline $8 \mathrm{~K}$ & & & & & \\
\hline $16 \mathrm{~K}$ & & & & & \\
\hline $32 \mathrm{~K}$ & & & & & \\
\hline $64 \mathrm{~K}$ & & & & & \\
\hline & & & & & \\
\hline & & & & & \\
\hline & & & & & \\
\hline & & & & & \\
\hline & & & 0 & & \\
\hline \multicolumn{6}{|c|}{ Unified Cache } \\
\hline$z e$ & & & & $8-$ & I0 \\
\hline & & & & & \\
\hline $2 \mathrm{~F}$ & & & & & \\
\hline 41 & & & & & \\
\hline 811 & & & & & \\
\hline & & & & & \\
\hline 20 & & & & & \\
\hline & & & & & \\
\hline in & & & & & \\
\hline & & & & & \\
\hline & & & & & \\
\hline $1 \mathrm{~N}$ & & & 0.0 & 0. & \\
\hline & & 0.0 & 0.0 & 0.00 & 0 \\
\hline
\end{tabular}

Table 11: Average Miss Ratios for Berkeley Multimedia Workload (128 byte Block Size) 
Berkeley Multimedia Workload

\begin{tabular}{|c|c|c|c|c|c|}
\hline & & & ultimedia & & \\
\hline & & nst. & cucti & ache & \\
\hline & & & Diat & & \\
\hline $\mathrm{ze}$ & Direct & & 4-way & 8-way & 16-wa \\
\hline & 0.00800 & $\begin{array}{l}0.01 \\
0.00\end{array}$ & 0.0 & & \\
\hline $4 \mathrm{~K}$ & & & & 0.00408 & \\
\hline & 0.0 & & 0.00 & 0.0 & \\
\hline & & & 0.00 & & \\
\hline $\begin{array}{l}4 \mathrm{~K} \\
8 \mathrm{~K}\end{array}$ & & 0.0 & 0.00 & 0.0 & \\
\hline$\left|\begin{array}{l}128 \mathrm{~K} \\
256 \mathrm{~K}\end{array}\right|$ & 0.00 & 0.00 & $\begin{array}{l}0.000007 \\
0\end{array}$ & 0.00007 & 0 \\
\hline $\begin{array}{l}56 \mathrm{~K} \\
12 \mathrm{~K}\end{array}$ & $\begin{array}{l}0.00 \\
0.00\end{array}$ & 0.000007 & 0.000 & $\begin{array}{l}0.00 \\
0.00\end{array}$ & \\
\hline 10 & & 0.00007 & 0.000 & 0.00007 & \\
\hline & & 0.00007 & 0.00007 & 0.00007 & 0.00 \\
\hline & & & & & \\
\hline & & & & & \\
\hline $2 \mathrm{~K}$ & & & & & \\
\hline & & & & & \\
\hline & 0.04 & 0.0 & 0.01 & $\begin{array}{c}18 \\
77\end{array}$ & \\
\hline k & & 0. & 0.0 & & \\
\hline $4 \mathrm{~K}$ & & & & & \\
\hline 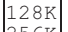 & & 0.0 & 0.0 & 0.0 & 0. \\
\hline $6 \mathrm{~K}$ & & & 0.00 & 0.0 & 0.0 \\
\hline 11 & & & 0.0 & $\begin{array}{c}39 \\
3\end{array}$ & $\begin{array}{l}0.0 \\
0.0\end{array}$ \\
\hline 1 & & 0.00 & 0.0 & & \\
\hline & & & & & \\
\hline & & & & $8-w c$ & 16-way \\
\hline & & & & & \\
\hline & & & & & \\
\hline & $\begin{array}{l}0.03 \\
0.01\end{array}$ & & $\begin{array}{l}0.9 \\
0.0\end{array}$ & 0. & \\
\hline & & & & & \\
\hline & 0.4 & & & & \\
\hline 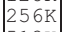 & 0. & & & & \\
\hline & & & & & \\
\hline & & & 0.0 & 0.0 & 0.00 \\
\hline & & & & & \\
\hline
\end{tabular}

\begin{tabular}{|c|c|c|c|c|c|}
\hline & & & Document & & \\
\hline & & Inst & action & ache & \\
\hline |Size| & irect & $\begin{array}{l}- \text {-way } \\
02355\end{array}$ & 4-way & 8-way & 16-way \\
\hline $2 \mathrm{~K}$ & 0.01826 & 0.01747 & 0.01823 & & \\
\hline & 0.01282 & 0.01121 & 0.01212 & 0.01119 & \\
\hline 8 & $\begin{array}{l}0.00797 \\
0.00365\end{array}$ & $\begin{array}{l}0.00607 \\
0.0234\end{array}$ & $\begin{array}{l}0.00617 \\
0.00105\end{array}$ & $\begin{array}{l}0.00444 \\
0.00015\end{array}$ & $\begin{array}{l}0.00471 \\
0.00015\end{array}$ \\
\hline & 0.00222 & 0.00012 & 0.00010 & 0.00007 & 0.00008 \\
\hline $\begin{array}{r}64 \mathrm{~K} \\
128 \mathrm{~K}\end{array}$ & 0.00215 & 0.00006 & $\begin{array}{l}0.00005 \\
0.0003\end{array}$ & 0.00005 & 0.00004 \\
\hline $256 \mathrm{~K}$ & 0.00090 & 0.00002 & 0.00002 & 0.00002 & 0.00002 \\
\hline & 0.00003 & 0.00002 & 0.00002 & 0.00002 & 0.00002 \\
\hline & 0.00002 & 0.00002 & 0.00002 & 0.00002 & 0.00002 \\
\hline & 0.00002 & 0.00002 & 0.00002 & 0.00002 & 0.00002 \\
\hline & & & $4-1$ & $8-17$ & 16 \\
\hline & & & 4-way & & $10-\mathrm{wa}$ \\
\hline & 21 & 682 & 0.11056 & & \\
\hline $\begin{array}{l}4 K \\
8 K\end{array}$ & $\begin{array}{l}0.088873 \\
0.06873\end{array}$ & $\begin{array}{l}0.0753 \\
0.0343\end{array}$ & {$\left[\begin{array}{l}0.05586 \\
0.02915\end{array}\right.$} & $\begin{array}{l}0.05178 \\
0.02038\end{array}$ & \\
\hline & 0.04088 & 0.02057 & 0.01536 & 0.01467 & 0.01435 \\
\hline & 56 & 0.01491 & 0.01372 & 0.01364 & 0.01363 \\
\hline $\begin{array}{r}64 \mathrm{~K} \\
128 \mathrm{~K}\end{array}$ & $\begin{array}{l}0.02365 \\
0.01601\end{array}$ & $\begin{array}{l}0.01366 \\
0.01325\end{array}$ & {$\left[\begin{array}{l}0.01327 \\
0.0137\end{array}\right]$} & $\begin{array}{l}0.01325 \\
0.01315\end{array}$ & $\begin{array}{l}0.01324 \\
0.01315\end{array}$ \\
\hline $256 \mathrm{~K}$ & 0.01353 & 0.01311 & 0.01310 & 0.01311 & 0.01311 \\
\hline $512 \mathrm{~K}$ & 0.00841 & 0.01172 & 0.01309 & 0.01309 & 0.01309 \\
\hline $1 \mathrm{M}$ & 0.04 & 0.00115 & 0.00114 & 0.00114 & 0.00114 \\
\hline & & & ified cand & $\frac{0 .}{e}$ & 0113 \\
\hline & & $\frac{2-n}{1}$ & 4 -way & 8-way & 16-way \\
\hline & & 0.0 & 0.05 & & \\
\hline & & & 0.03361 & & \\
\hline & $\begin{array}{r}.04340 \\
.02345\end{array}$ & 0.01352 & $\begin{array}{l}0.01885 \\
0.0096\end{array}$ & $\begin{array}{l}0.01664 \\
0.00691\end{array}$ & $\begin{array}{l}0.01584 \\
0.00735\end{array}$ \\
\hline 32 & 0.0 & 0.0 & 0.00439 & 0.00309 & \\
\hline 64. & 0.01132 & 0.00 & 0.00289 & 0.00286 & 0.00285 \\
\hline $\begin{array}{c}28 \mathrm{~K} \\
56 \mathrm{~K}\end{array}$ & & $\begin{array}{l}0.00 \\
0.00\end{array}$ & $\begin{array}{l}0.00280 \\
0.00275\end{array}$ & $\begin{array}{l}0.00278 \\
0.00275\end{array}$ & \\
\hline & & & 0.00274 & 0.00274 & 0.00274 \\
\hline & 0 & 0.00026 & 0.00025 & 0.00025 & $\begin{array}{l}0.00025 \\
0.0002\end{array}$ \\
\hline & & & & & \\
\hline
\end{tabular}
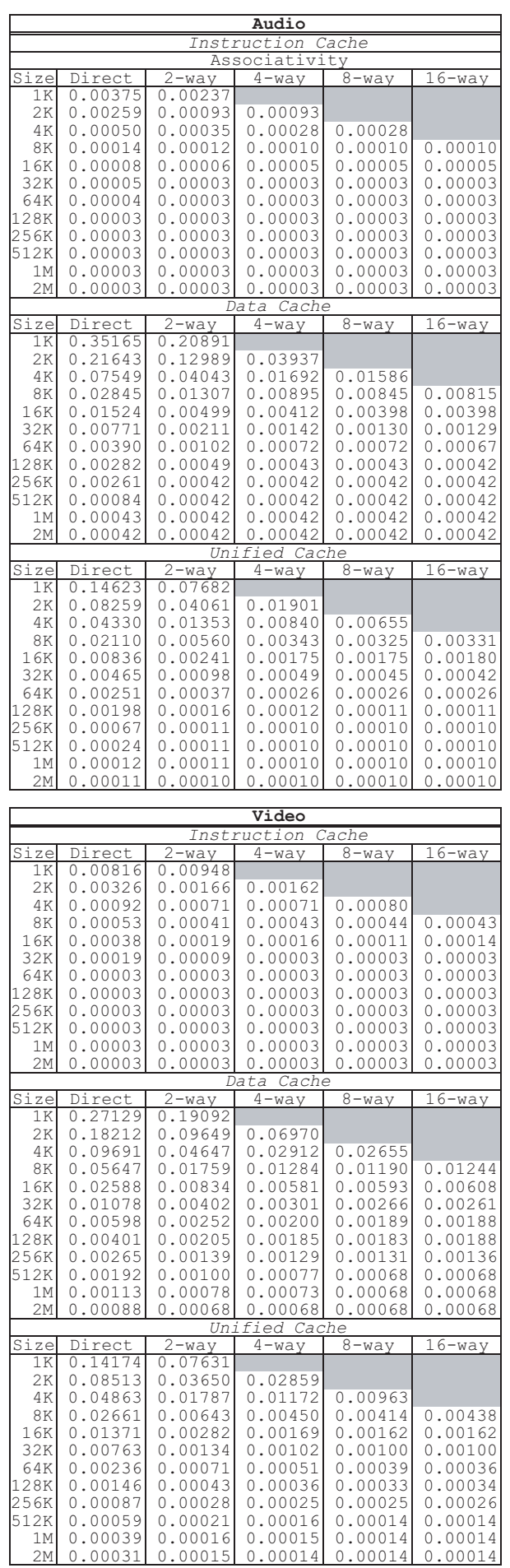

Block Size: 256 bytes Speech

\begin{tabular}{|c|c|c|c|c|c|}
\hline \multicolumn{6}{|c|}{ che } \\
\hline \multicolumn{6}{|c|}{ Associativity } \\
\hline Size & Direct & 2-way & 4-way & $8-w a y$ & 16-way \\
\hline $1 \mathrm{~K}$ & 0.02492 & 0.01613 & & & \\
\hline $2 \mathrm{~K}$ & 0.00663 & 0.00575 & 0.00603 & & \\
\hline $4 \mathrm{~K}$ & 0.00429 & 0.00381 & 0.00341 & 0.00338 & \\
\hline $8 \mathrm{~K}$ & 0.00302 & 0.00274 & 0.00276 & 0.00278 & 0.00278 \\
\hline $16 \mathrm{~K}$ & 0.00162 & 0.00127 & 0.00123 & 0.00078 & 055 \\
\hline $32 \mathrm{~K}$ & 0.00078 & 0.00049 & 0.00040 & 0.00043 & 0.00043 \\
\hline $64 \mathrm{~K}$ & 0.00044 & 0.00025 & 0.00024 & 0.00018 & 0.00018 \\
\hline $128 \mathrm{~K}$ & 0.00024 & 0.00020 & 0.00018 & 0.00018 & 0.00018 \\
\hline $256 \mathrm{~K}$ & 0.00021 & 0.00018 & 0.00018 & 0.00018 & 0.00018 \\
\hline $512 \mathrm{~K}$ & 0.00018 & 0.00018 & 0.00018 & 0.00018 & 0.00018 \\
\hline $1 \mathrm{M}$ & 0.00018 & 0.00018 & 0.00018 & 0.00018 & 0.00018 \\
\hline $2 \mathrm{M}$ & 0.00018 & 0.00018 & 0.00018 & 0.00018 & 0.00018 \\
\hline \multicolumn{6}{|c|}{ Data Cache } \\
\hline ize & Direct & 2-way & 4-way & 8-way & 16-way \\
\hline $1 \mathrm{~K}$ & 0.27453 & 0.12264 & & & \\
\hline $2 \mathrm{~K}$ & 0.22725 & 0.06121 & 0.04562 & & \\
\hline $4 \mathrm{~K}$ & 0.05611 & 0.02604 & 0.01822 & 0.01281 & \\
\hline $8 \mathrm{~K}$ & 0.02781 & 0.01160 & 0.00643 & 0.00514 & 0.00425 \\
\hline $16 \mathrm{~K}$ & 0.01590 & 0.00328 & 0.00231 & 0.00208 & 0.00199 \\
\hline $32 \mathrm{~K}$ & 0.00686 & 0.00185 & 0.00179 & 0.00179 & 0.00179 \\
\hline $64 \mathrm{~K}$ & 0.00544 & 0.00121 & 0.00108 & 0.00097 & 0.00096 \\
\hline $128 \mathrm{~K}$ & 0.00163 & 0.00074 & 0.00065 & 0.00050 & 0.00050 \\
\hline $256 \mathrm{~K}$ & 0.00065 & 0.00054 & 0.00050 & 0.00049 & 0.00049 \\
\hline $512 \mathrm{~K}$ & 0.00052 & 0.00049 & 0.00049 & 0.00049 & 0.00049 \\
\hline $1 \mathrm{M}$ & 0.00050 & 0.00049 & 0.00049 & 0.00049 & 0.00049 \\
\hline $2 \mathrm{M}$ & 0.00050 & 0.00049 & 0.00049 & 0.00049 & 0.00049 \\
\hline \multicolumn{6}{|c|}{ Unified Cache } \\
\hline Size & Direct & $2-$ & 4-way & $8-w a y$ & 16-way \\
\hline $1 \mathrm{~K}$ & 0.1 & 0.08478 & & & \\
\hline $2 \mathrm{~K}$ & 0.10520 & 0.04863 & 0.03381 & & \\
\hline $4 \mathrm{~K}$ & 0.05250 & 0.02795 & 0.01712 & 0.01600 & \\
\hline $8 \mathrm{~K}$ & 0.02824 & 0.00835 & 0.00685 & 0.00534 & 0.00486 \\
\hline $16 \mathrm{~K}$ & 0.02089 & 0.00384 & 0.00316 & 0.00326 & 0.00332 \\
\hline $32 \mathrm{~K}$ & 0.01580 & 0.00186 & 0.00119 & 0.00099 & 0.00091 \\
\hline $64 \mathrm{~K}$ & 0.01338 & 0.00091 & 0.00076 & 0.00077 & 0.00077 \\
\hline $128 \mathrm{~K}$ & 0.00191 & 0.00051 & 0.00041 & 0.00032 & 0.00030 \\
\hline $256 \mathrm{~K}$ & 0.00157 & 0.00029 & 0.00027 & 0.00025 & 0.00024 \\
\hline $512 \mathrm{~K}$ & 0.00046 & 0.00025 & 0.00024 & 0.00024 & 0.00024 \\
\hline $1 \mathrm{M}$ & 0.00045 & 0.00024 & 0.00024 & 0.00024 & 0.00024 \\
\hline $2 \mathrm{M}$ & 0.00045 & 0.00024 & 0.00024 & 0.00024 & 0.00024 \\
\hline
\end{tabular}

3D

\begin{tabular}{|c|c|c|c|c|c|}
\hline \multicolumn{6}{|c|}{ che } \\
\hline Size & Direct & 2-way & $4-w a y$ & $8-$ way & 16-way \\
\hline $1 \mathrm{~K}$ & 0.01405 & 0.01291 & & & \\
\hline $2 \mathrm{~K}$ & 0.00928 & 0.00884 & 0.00852 & & \\
\hline $4 \mathrm{~K}$ & 0.00595 & 0.00514 & 0.00478 & 0.00478 & \\
\hline $8 \mathrm{~K}$ & 0.00402 & 0.00306 & 0.00253 & 0.00243 & 0.00249 \\
\hline $16 \mathrm{~K}$ & 0.00281 & 0.00187 & 0.00181 & 0.00185 & 0187 \\
\hline $32 \mathrm{~K}$ & 0.00173 & 0.00104 & 0.00080 & 0.00080 & 0.00084 \\
\hline $64 \mathrm{~K}$ & 0.00096 & 0.00034 & 0.00017 & 0.00011 & 0.00008 \\
\hline $128 \mathrm{~K}$ & 0.00063 & 0.00007 & 0.00006 & 0.00006 & 0.00006 \\
\hline $256 \mathrm{~K}$ & 0.00040 & 0.00007 & 0.00006 & 0.00006 & 0.00006 \\
\hline $512 \mathrm{~K}$ & 0.00029 & 0.00006 & 0.00006 & 0.00006 & 0.00006 \\
\hline $1 \mathrm{M}$ & 0.00008 & 0.00006 & 0.00006 & 0.00006 & 0.00006 \\
\hline $2 \mathrm{M}$ & 0.00006 & 0.00006 & 0.00006 & 0.00006 & 0.00006 \\
\hline \multicolumn{6}{|c|}{ Data Cache } \\
\hline Size & Direct & 2-way & 4-way & 8-way & 16-way \\
\hline $1 \mathrm{~K}$ & 0.24001 & 0.18411 & & & \\
\hline $2 \mathrm{~K}$ & 0.15936 & 0.10184 & 0.08182 & & \\
\hline $4 \mathrm{~K}$ & 0.10310 & 0.06481 & 0.05106 & 0.04976 & \\
\hline $8 \mathrm{~K}$ & 0.06736 & 0.04324 & 0.03678 & 0.03506 & 0.03374 \\
\hline $16 \mathrm{~K}$ & 0.04576 & 0.03150 & 0.02810 & 0.02718 & 0.02683 \\
\hline $32 \mathrm{~K}$ & 0.02812 & 0.01936 & 0.01770 & 0.01764 & 0.01742 \\
\hline $64 \mathrm{~K}$ & 0.01736 & 0.01068 & 0.00924 & 0.00877 & 0.00863 \\
\hline $128 \mathrm{~K}$ & 0.00957 & 0.00754 & 0.00663 & 0.00650 & 0.00647 \\
\hline $256 \mathrm{~K}$ & 0.00728 & 0.00565 & 0.00552 & 0.00557 & 0.00561 \\
\hline $512 \mathrm{~K}$ & 0.00574 & 0.00485 & 0.00478 & 0.00477 & 0.00476 \\
\hline $1 \mathrm{M}$ & 0.00500 & 0.00450 & 0.00443 & 0.00442 & 0.00442 \\
\hline $2 \mathrm{M}$ & 0.00481 & 0.00435 & 0.00432 & 0.00431 & 0.00431 \\
\hline \multicolumn{6}{|c|}{ Unified Cache } \\
\hline Size & Dir & $2-w a$ & 4-way & 8-way & 16-way \\
\hline & 775 & 0.09518 & & & \\
\hline $2 \mathrm{~K}$ & 0.12 & 0.05406 & 0 . & & \\
\hline $4 K$ & 0.07513 & 0.03149 & 0.02438 & 0.02391 & \\
\hline $8 \mathrm{~K}$ & 0.03529 & 0.01937 & 0.01666 & 0.01567 & 0.01585 \\
\hline $16 \mathrm{~K}$ & 0.02286 & 0.01217 & 0.01084 & 0.01002 & 0.00979 \\
\hline $32 \mathrm{~K}$ & 0.01362 & 0.00794 & 0.00705 & 0.00688 & 0.00695 \\
\hline $64 \mathrm{~K}$ & 0.00868 & 0.00459 & 0.00393 & 0.00370 & 0.00372 \\
\hline $128 \mathrm{~K}$ & 0.00527 & 0.00266 & 0.00205 & 0.00183 & 0.001 \\
\hline $256 \mathrm{~K}$ & 0.00387 & 0.00170 & 0.00141 & 0.00135 & 0.00134 \\
\hline $512 \mathrm{~K}$ & 0.00259 & 0.00128 & 0.00115 & 0.00115 & 0.0011 \\
\hline $1 \mathrm{M}$ & 0.00203 & 0.00108 & 0.00105 & 0.00105 & 0.0010 \\
\hline $2 \mathrm{M}$ & 0.00194 & 0.00103 & 0.0010 & 0.0010 & 0.00102 \\
\hline
\end{tabular}

Table 12: Average Miss Ratios for Berkeley Multimedia Workload (256 byte Block Size) 

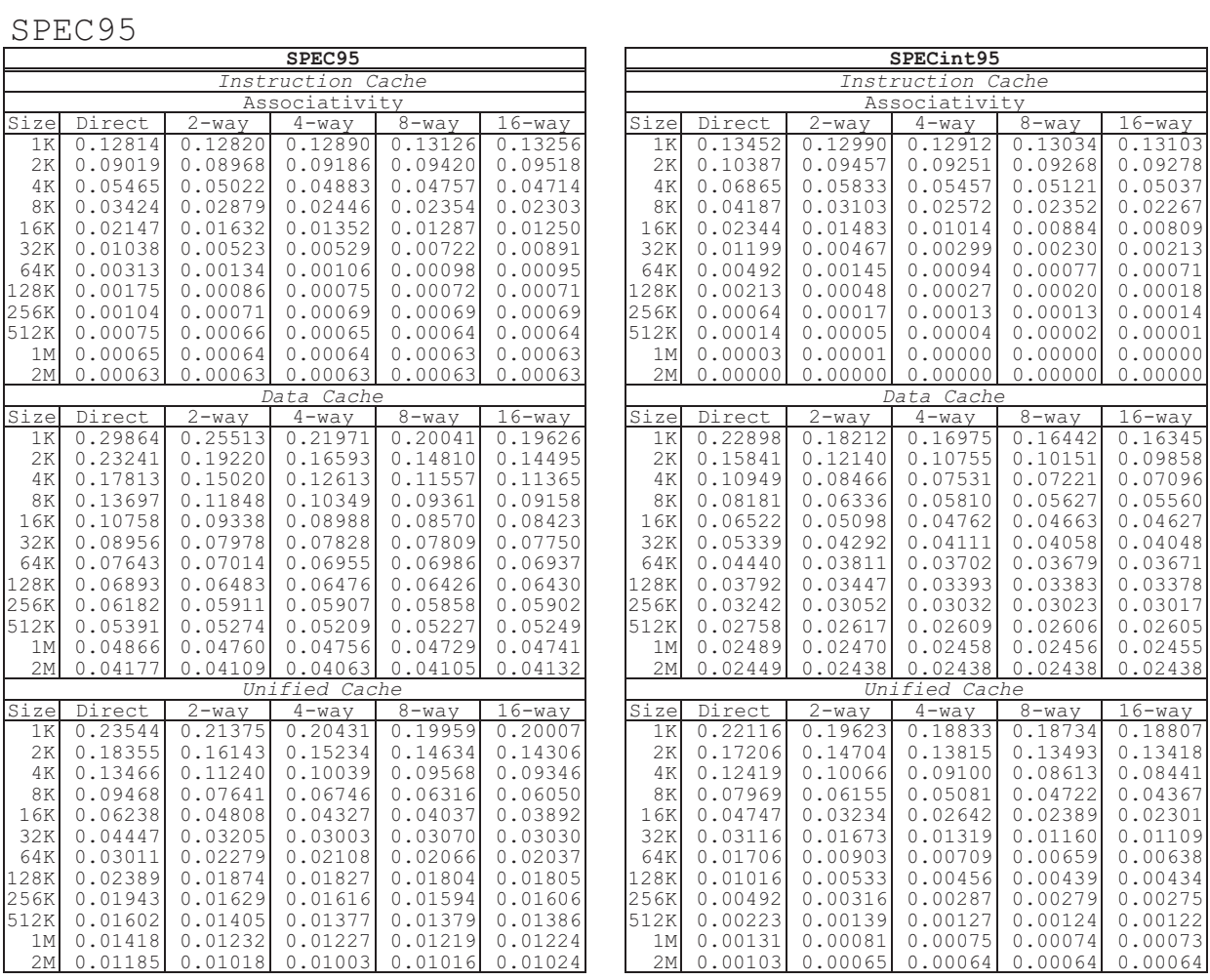

Block Size: 16 bytes SPECfp95

\begin{tabular}{|c|c|c|c|c|c|}
\hline & & & 095 & & \\
\hline & & $\frac{\overline{I n s t r}}{\mathrm{Ass}}$ & $\begin{array}{l}\text { ction C } \\
\text { ciativi }\end{array}$ & he & \\
\hline 101 & T) & & & 8-way & $\frac{16-\mathrm{wc}}{\frac{132}{132}}$ \\
\hline 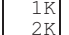 & $\begin{array}{l}0.12 \\
0.07\end{array}$ & $\begin{array}{l}0.12684 \\
0.08576\end{array}$ & $\begin{array}{l}0.12873 \\
0.09134\end{array}$ & $\begin{array}{l}0.13200 \\
0.09541\end{array}$ & \\
\hline & & & & & \\
\hline & & & & & \\
\hline & & & & & \\
\hline & & & & & \\
\hline & & & & 0.00 & \\
\hline $\begin{array}{l}8 \mathrm{~B} \\
6 \mathrm{~K}\end{array}$ & $\begin{array}{l}0.00 \\
0.00\end{array}$ & 4. & $\begin{array}{l}4 \\
4\end{array}$ & 0.00114 & 0. \\
\hline $2 \mathrm{~K}$ & 0.00 & 0.00114 & 0.00114 & 0.00114 & \\
\hline & & 0.00114 & 0.00114 & & \\
\hline & & 0.00114 & 0.00114 & 0.00114 & 0.0011 \\
\hline & & & & & \\
\hline & & & & & \\
\hline & & & & & \\
\hline & & & & & \\
\hline & 0.1 & & & & \\
\hline & & 0.12 & & 0.1 & o. \\
\hline 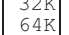 & 0.1 & & & & \\
\hline $28 \mathrm{~K}$ & 0.0 & 0. & & & \\
\hline 1 & 0.0 & & & & \\
\hline 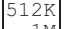 & & & & & \\
\hline 11 & & & & & \\
\hline & & & 0.0 & & \\
\hline $\mathrm{ze}$ & & & & & \\
\hline & & & & & \\
\hline & & & & & \\
\hline 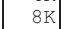 & 0.1 & & & & \\
\hline & & & & & \\
\hline & & & & & \\
\hline & & & & & \\
\hline & & & & & \\
\hline 256 & & & & & \\
\hline 512 & & & & & \\
\hline & & & & & \\
\hline & & & & & \\
\hline
\end{tabular}

\section{SPEC95}

\begin{tabular}{|c|c|c|c|c|c|}
\hline & & & SPI & & \\
\hline & & 1710 & & & \\
\hline & & 2-way & & -wa & 6-wa \\
\hline & $\begin{array}{l}0.07052 \\
0.0499\end{array}$ & $\begin{array}{l}0.07013 \\
0.0489\end{array}$ & $\begin{array}{l}25 \\
23\end{array}$ & $\begin{array}{l}0.07159 \\
0.05136\end{array}$ & 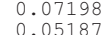 \\
\hline & 0.03025 & 0.02813 & 0.0 & 0.02661 & 0.02634 \\
\hline & & 0015 & & 001337 & \\
\hline & & & & & \\
\hline $32 \mathrm{~F}$ & 0.00 & 0.00282 & 0.00260 & 0.00352 & 00435 \\
\hline & & 0.00 & & $\begin{array}{l}.00022 \\
0.00005\end{array}$ & \\
\hline $56 \mathrm{~F}$ & & & & & \\
\hline & & & & & \\
\hline & & & & & \\
\hline & & & $\frac{0.00}{\operatorname{tac} c}$ & & \\
\hline & & & & & \\
\hline & $.283^{3}$ & & & & \\
\hline & & & & & \\
\hline & & & & & \\
\hline & 4 & & & & \\
\hline & & & & & \\
\hline & 0.0 & 6] & $\begin{array}{l}0.04 \\
0.03\end{array}$ & 0.0 & \\
\hline & & & 0.0 & & \\
\hline $66 \mathrm{~K}$ & & & & & \\
\hline $2 \mathrm{~K}$ & 0.0 & & 0.0 & 0.0 & \\
\hline & & & 0.01 & 0.0 & 0.0 \\
\hline & & & & & \\
\hline & & & & & \\
\hline & & & & & \\
\hline & 0. & & & & \\
\hline & & & & & \\
\hline & & & & & \\
\hline & & & & & \\
\hline & & & & & \\
\hline & & & & & \\
\hline & & & & & \\
\hline & & & 0.00507 & 0.00513 & 0.00 \\
\hline
\end{tabular}

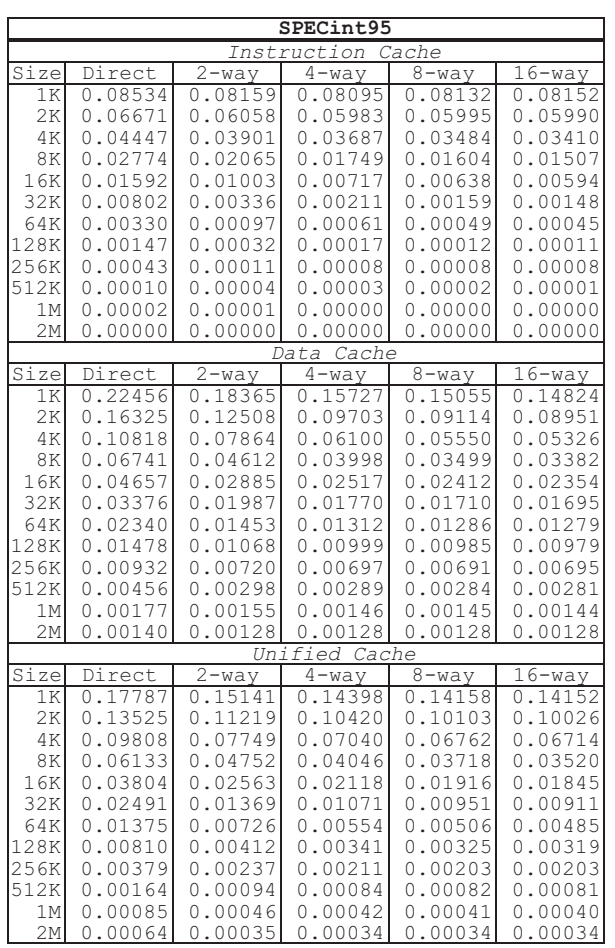

Block Size: 32 bytes

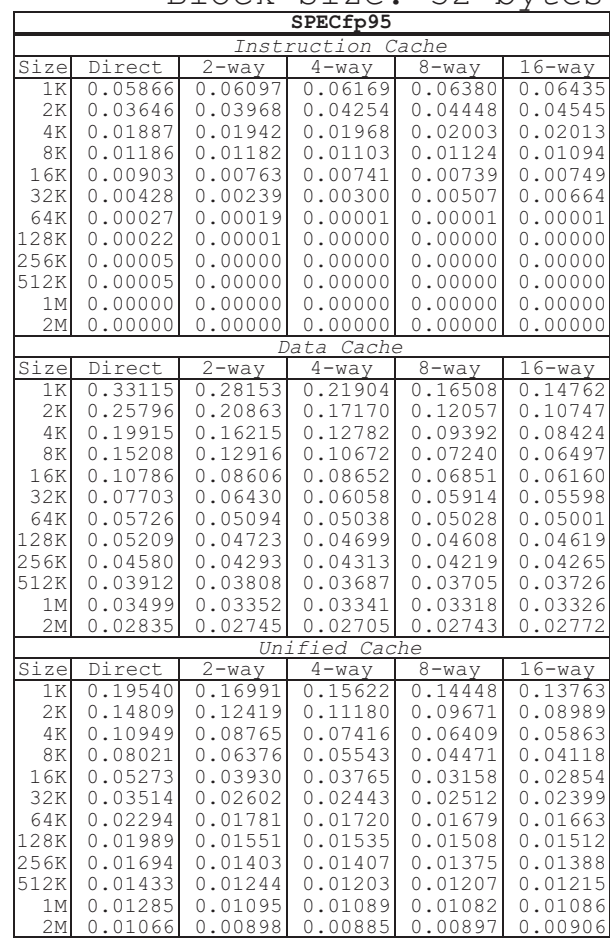

Table 13: Arithmetic Average Miss Ratios for SPEC95 (16, 32 byte Block Sizes) 

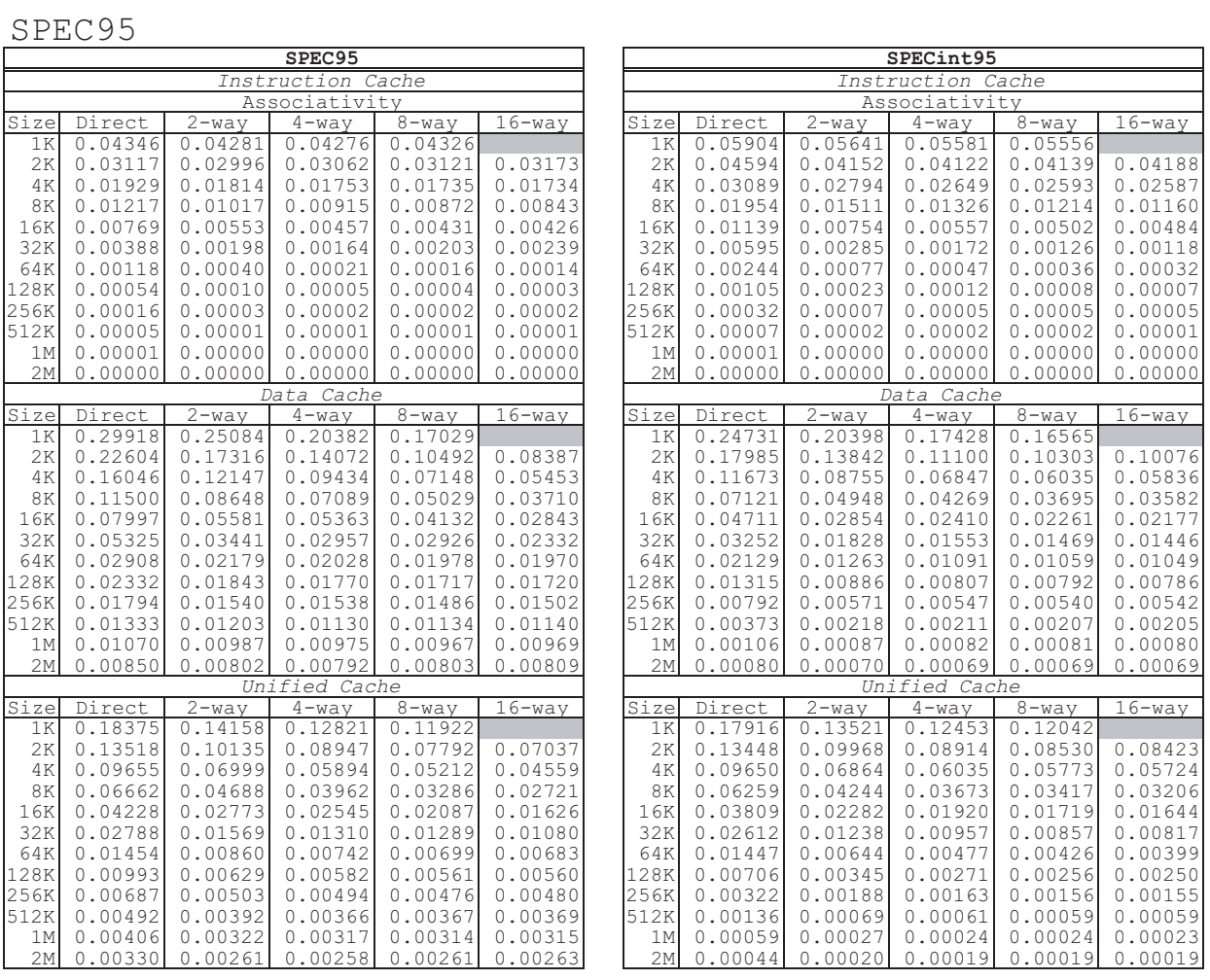

Block Size: 64 bytes

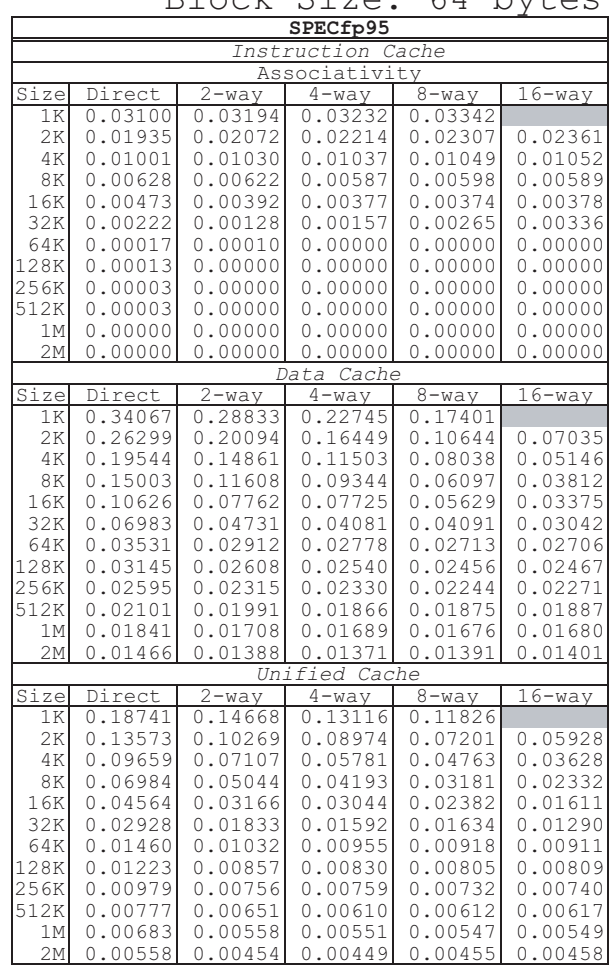

SPEC95

\begin{tabular}{|c|c|c|c|c|c|}
\hline & & & & & \\
\hline & & & on 0 & & \\
\hline & & 2-way & & 8-way & 16-way \\
\hline & $\begin{array}{l}0.03940 \\
0.02769\end{array}$ & $\begin{array}{l}0.03553 \\
0.02332\end{array}$ & & 0.02322 & \\
\hline & 0.01764 & 0.01428 & 0.01322 & & 0.01313 \\
\hline & 0.00987 & 0.00802 & 0.00705 & 684 & \\
\hline $16 \mathrm{~K}$ & 0.0 & 0.00421 & 37 & 319 & \\
\hline & 0.00353 & 0.00184 & 0.00138 & 156 & 66 \\
\hline & 0.00 & 0.00066 & & & \\
\hline $28 \mathrm{~K}$ & 0.00 & 0.00 & 0.00 & & 0.00 \\
\hline $12 \mathrm{~K}$ & ${ }_{0}^{0}$. & 0. & 0.00 & 0. & 0. \\
\hline & 0.0 & & 0.0 & & \\
\hline & & & & & \\
\hline & & & & & \\
\hline & & & & 8-way & 16-way \\
\hline & 0.2 & & & 44 & \\
\hline & 0.18 & & & & 0. \\
\hline & & & & & \\
\hline & & & & & \\
\hline & & & & & \\
\hline & & & & & \\
\hline & 0.0 & 0.0 & 0.0 & 0. & \\
\hline & 0.0 & & & & \\
\hline $12 \mathrm{~K}$ & 0.008 & 0.00 & 0.00602 & 0.00 & 0.00607 \\
\hline & 0.00 & 0.00 & 0.00 & 0.00492 & 0.00493 \\
\hline & & & $\frac{4021}{\mathrm{CaCl}}$ & $\frac{0.00406}{h e}$ & 0.00409 \\
\hline & & & & $8-w$ & 16-way \\
\hline & & & & & \\
\hline & & & 8 & & \\
\hline & & & & & \\
\hline & & & & & \\
\hline & & $\begin{array}{l}0.0 \\
0.0\end{array}$ & & $\begin{array}{l}0.0 \\
0.0\end{array}$ & \\
\hline & 0.0 & & 0.0 & & \\
\hline & & & & & \\
\hline & & & & & \\
\hline & & & & & \\
\hline & & & & & \\
\hline
\end{tabular}

\begin{tabular}{|c|c|c|c|c|c|}
\hline & & & SPECint95 & & \\
\hline & & Inst. & ruction $\mathrm{C}$ & ache & \\
\hline ize & & & & 8-way & 16-way \\
\hline & 0.06695 & & 0.0564 & & \\
\hline $2 \mathrm{~K}$ & $\begin{array}{l}0.04850 \\
0.03270\end{array}$ & $\begin{array}{l}0.03826 \\
0.02509\end{array}$ & $\begin{array}{ll}154 \\
776 \\
\end{array}$ & & \\
\hline & 0.01 & 0.01 & 0.0 & 0.0 & \\
\hline $16 \mathrm{~K}$ & 0.0 & 0.00691 & 0.0 & 0.00475 & 59 \\
\hline & 0 & $\begin{array}{r}0.00323 \\
0.00142\end{array}$ & 0.00206 & 0.00177 & $\begin{array}{l}613 \\
83 \\
\end{array}$ \\
\hline & & & & & \\
\hline $256 \mathrm{~K}$ & & 000 & 0.08 & 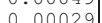 & \\
\hline $512 \mathrm{~K}$ & 0.0 & 0.00024 & 0.00021 & 0.00020 & \\
\hline & 0.0 & 0.00016 & 0.00014 & 0.00014 & .0002 \\
\hline & 0.0 & 0.00010 & 0.00009 & 0.00009 & 0.000 \\
\hline & & & & & \\
\hline & & & & & \\
\hline & & & & & \\
\hline & & & & & \\
\hline & & & & & 06906 \\
\hline & 0.08 & & 0.0 & & \\
\hline & 0.05 & & & & \\
\hline & 0 & & 0.0 & 4 & .0 \\
\hline $128 \mathrm{~K}$ & 02 & & 0.0 & 0.08 & 0.0 \\
\hline $256 \mathrm{~K}$ & (1) & 0 & 0.0 & 0.00 & 0.0 \\
\hline $2 \mathrm{~K}$ & & & 0.0 & 0.00 & .0 \\
\hline $1 \mathrm{M}$ & & & 0.0 & 0.0 & 0.0 \\
\hline & 0.0 & 0. & 0 . & 0.00 & 0.0003 \\
\hline & & & & & \\
\hline & & & & $8-w e$ & 16-way \\
\hline & & & & & \\
\hline & & & & & \\
\hline & 9 & & & & \\
\hline & & & & & \\
\hline & & & & & \\
\hline & & & & & \\
\hline & 0.02 a & 0. & & & \\
\hline 512 & & & & & \\
\hline & & & & & \\
\hline & & & & & \\
\hline
\end{tabular}

Block Size: 128 bytes

\begin{tabular}{|c|c|c|c|c|c|}
\hline & & & SPECfp95 & & \\
\hline & & & ruction C & & \\
\hline $1 \mathrm{KK}$ & 01735 & 0 & $\frac{4-\text { way }}{0.1816}$ & 8-way & 16-way \\
\hline & $\begin{array}{l}0.01735 \\
0.01104\end{array}$ & $\begin{array}{l}0.01760 \\
0.01137\end{array}$ & $\left|\begin{array}{l}0.01816 \\
0.01241\end{array}\right|$ & 0.01283 & \\
\hline & 0.00559 & 0.00563 & 0.00559 & & 005 \\
\hline & 0.00346 & 0.00341 & 0.00328 & & \\
\hline $16 \mathrm{~K}$ & 0.00252 & 0.00206 & 0.00196 & & \\
\hline & 0.00118 & 0.00072 & 0.0 & & \\
\hline & & & & & \\
\hline $\mid \begin{array}{l}128 \mathrm{~K} \\
256 \mathrm{~K}\end{array}$ & 0.0 & & 0.00 & & \\
\hline $\begin{array}{l}512 \mathrm{~K} \\
51\end{array}$ & . & 0.0 & 0.0 & 0. & 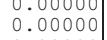 \\
\hline & & & & & \\
\hline & & & 0.00 & 0.00000 & 0.000 \\
\hline & & & & $8-w$ & -wa \\
\hline & & & & & \\
\hline & & & & 0.12 & \\
\hline & & & & & \\
\hline & & & & & \\
\hline & & & & & \\
\hline & & 4 & & & \\
\hline 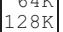 & & & 0.0 - & 0. & \\
\hline $256 \mathrm{~K}$ & & 2 & & 0.0 & 0.0 \\
\hline $512 \mathrm{~K}$ & & 0 & 0.0 & 0.00 & 0.0 \\
\hline & & 0.008 & 0.00 & 0.00847 & 0.008 \\
\hline & & & $\frac{0.0}{f i e}$ & & \\
\hline & & & & $8-$ & $6-\mathrm{w}$ \\
\hline & & & & & \\
\hline & & & & & \\
\hline & & & & & \\
\hline & & & & & \\
\hline $\begin{array}{l}32 \mathrm{~K} \\
64 \mathrm{~K}\end{array}$ & 0.0 & & 0.00 & & \\
\hline & & & & & \\
\hline & & & & & \\
\hline 512 & & & 0.0 & & \\
\hline & & & .00227 & 00229 & .00 \\
\hline
\end{tabular}

Table 14: Arithmetic Average Miss Ratios for SPEC95 (64, 128 byte Block Sizes) 


\begin{tabular}{|c|c|c|c|c|c|}
\hline \multicolumn{6}{|c|}{ SPEC95 } \\
\hline \multirow{2}{*}{\multicolumn{6}{|c|}{$\begin{array}{c}\text { Instruction Cache } \\
\text { Associativity }\end{array}$}} \\
\hline & & & & & \\
\hline Size & Direct & 2-way & 4-way & 8-way & 16-way \\
\hline $1 \mathrm{~K}$ & 0.02236 & 0.02121 & & & \\
\hline $2 \mathrm{~K}$ & 0.01619 & 0.01396 & 0.01396 & & \\
\hline $4 \mathrm{~K}$ & 0.01040 & 0.00914 & 0.00883 & 0.00893 & \\
\hline $8 \mathrm{~K}$ & 0.00623 & 0.00533 & 0.00513 & 0.00507 & 0.00496 \\
\hline $16 \mathrm{~K}$ & 0.00391 & 0.00288 & 0.00238 & 0.00225 & 0.00215 \\
\hline $32 \mathrm{~K}$ & 0.00204 & 0.00124 & 0.00097 & 0.00097 & 0.00102 \\
\hline $64 \mathrm{~K}$ & 0.00072 & 0.00037 & 0.00019 & 0.00014 & 0.00012 \\
\hline $128 \mathrm{~K}$ & 0.00029 & 0.00007 & 0.00004 & 0.00003 & 0.00002 \\
\hline $256 \mathrm{~K}$ & 0.00009 & 0.00002 & 0.00001 & 0.00001 & 0.00001 \\
\hline $512 \mathrm{~K}$ & 0.00003 & 0.00001 & 0.00001 & 0.00001 & 0.00001 \\
\hline $1 \mathrm{M}$ & 0.00000 & 0.00000 & 0.00000 & 0.00000 & 0.00000 \\
\hline $2 \mathrm{M}$ & 0.00000 & 0.00000 & 0.00000 & 0.00000 & 0.00000 \\
\hline \multicolumn{6}{|c|}{ ta } \\
\hline $\mathrm{ze}$ & Direct & 2-way & 4-way & 8-way & vay \\
\hline $1 \mathrm{~K}$ & 0.41667 & 0.36569 & & & \\
\hline $2 \mathrm{~K}$ & 0.30412 & 0.24760 & 0.21125 & & \\
\hline $4 \mathrm{~K}$ & 0.21680 & 0.16523 & 0.12738 & 0.10471 & \\
\hline $8 \mathrm{~K}$ & 0.15203 & 0.11208 & 0.08499 & 0.06067 & 0.03559 \\
\hline $16 \mathrm{~K}$ & 0.10690 & 0.07543 & 0.06130 & 0.04592 & 0.02279 \\
\hline $32 \mathrm{~K}$ & 0.07109 & 0.04624 & 0.02767 & 0.02480 & 0.01341 \\
\hline $64 \mathrm{~K}$ & 0.03 & 0.02765 & 0.01280 & 0.01040 & 0.00947 \\
\hline $128 \mathrm{~K}$ & 0.01758 & 0.01060 & 0.00993 & 0.00749 & 0.00753 \\
\hline $256 \mathrm{~K}$ & 0.01 & 0.00668 & 0.00654 & 0.00605 & 0.00611 \\
\hline $512 \mathrm{~K}$ & 0.00633 & 0.00412 & 0.00338 & 0.00338 & 0.00341 \\
\hline $1 \mathrm{M}$ & 0.00363 & 0.00272 & 0.00257 & 0.00253 & 0.00254 \\
\hline $2 \mathrm{M}$ & 0.00260 & 0.00209 & 0.00206 & 0.00207 & 0.00208 \\
\hline \multicolumn{6}{|c|}{ Unified Cache } \\
\hline Size & Direct & 2-way & 4-way & 8-way & 16-way \\
\hline $1 \mathrm{~K}$ & 0.28138 & 0.17229 & & & \\
\hline $2 \mathrm{~K}$ & 0.19118 & 0.11599 & 0.10091 & & \\
\hline $4 \mathrm{~K}$ & 0.13093 & 0.07715 & 0.06237 & 0.0 & \\
\hline $8 \mathrm{~K}$ & 0.07943 & 0.05023 & 0.03877 & 0.03066 & 0.02233 \\
\hline $16 \mathrm{~K}$ & 0.05024 & 0.03143 & 0.02595 & 0.02053 & 0.01248 \\
\hline $32 \mathrm{~K}$ & 0.03393 & 0.01835 & 0.01186 & 0.01074 & 0.00680 \\
\hline $64 \mathrm{~K}$ & 0.01741 & 0.01029 & 0.00511 & 0.00410 & 0.00368 \\
\hline $128 \mathrm{~K}$ & 0.00795 & 0.00379 & 0.00331 & 0.00243 & 0.00242 \\
\hline$\rho_{0}-\sigma^{\prime}$ & 0.0 & 0.0 & 0.00207 & 0.00190 & 0.00191 \\
\hline $512 \mathrm{~K}$ & 0.00244 & 0.00135 & 0.00109 & 0.00109 & 0.00110 \\
\hline $1 \mathrm{M}$ & 0.0 & 0.0 & 0.00084 & 0.00083 & 0.00083 \\
\hline $0 \mathrm{~s}$ & 0.0 & 0.00068 & 00007 & 0.00067 & 0.00068 \\
\hline
\end{tabular}

\begin{tabular}{|c|c|c|c|c|c|}
\hline \multicolumn{6}{|c|}{ SPECint95 } \\
\hline \multirow{2}{*}{\multicolumn{6}{|c|}{$\frac{\text { Instruction Cache }}{\text { Associativity }}$}} \\
\hline & & & & & \\
\hline Size & Direct & 2-way & 4-way & 8-way & 16-way \\
\hline $1 \mathrm{~K}$ & 0.03692 & 0.03401 & & & \\
\hline $2 \mathrm{~K}$ & 0.02813 & 0.02341 & 0.02265 & & \\
\hline $4 \mathrm{~K}$ & 0.01896 & 0.01640 & 0.01593 & 0.01610 & \\
\hline $8 \mathrm{~K}$ & 0.01115 & 0.00957 & 0.00916 & 0.00895 & 0.00867 \\
\hline $16 \mathrm{~K}$ & 0.00665 & 0.00503 & 0.00401 & 0.00373 & 0.00352 \\
\hline $32 \mathrm{~K}$ & 0.00375 & 0.00224 & 0.00160 & 0.00124 & 0.00119 \\
\hline $64 \mathrm{~K}$ & 0.00151 & 0.00079 & 0.00043 & 0.00031 & 0.00026 \\
\hline $128 \mathrm{~K}$ & 0.00059 & 0.00016 & 0.00008 & 0.00006 & 0.00005 \\
\hline $256 \mathrm{~K}$ & 0.00019 & 0.00004 & 0.00002 & 0.00002 & 0.00002 \\
\hline $512 \mathrm{~K}$ & 0.00005 & 0.00001 & 0.00001 & 0.00001 & 0.00001 \\
\hline $1 \mathrm{M}$ & 0.00001 & 0.00000 & 0.00000 & 0.00000 & 0.00000 \\
\hline $2 \mathrm{M}$ & 0.00000 & 0.00000 & 0.00000 & 0.00000 & 0.00000 \\
\hline \multicolumn{6}{|c|}{ Data Cache } \\
\hline \begin{tabular}{|l|} 
Size \\
\end{tabular} & Direct & 2-way & 4-way & 8-way & 16-way \\
\hline $1 \mathrm{~K}$ & 0.35612 & 0.30264 & & & \\
\hline $2 \mathrm{~K}$ & 0.25111 & 0.20744 & 0.17771 & & \\
\hline $4 \mathrm{~K}$ & 0.16943 & 0.12624 & 0.10715 & 0.09882 & \\
\hline $8 \mathrm{~K}$ & 0.10831 & 0.07599 & 0.06561 & 0.05567 & 0.05192 \\
\hline $16 \mathrm{~K}$ & 0.07119 & 0.04124 & 0.03438 & 0.03248 & 0.03064 \\
\hline $32 \mathrm{~K}$ & 0.04689 & 0.02272 & 0.01806 & 0.01616 & 0.01562 \\
\hline $64 \mathrm{~K}$ & 0.03068 & 0.01348 & 0.00987 & 0.00907 & 0.00881 \\
\hline $128 \mathrm{~K}$ & 0.01603 & 0.00818 & 0.00676 & 0.00648 & 0.00644 \\
\hline $256 \mathrm{~K}$ & 0.00899 & 0.00470 & 0.00437 & 0.00427 & 0.00426 \\
\hline $512 \mathrm{~K}$ & 0.00465 & 0.00159 & 0.00152 & 0.00149 & 0.00147 \\
\hline $1 \mathrm{M}$ & 0.00061 & 0.00036 & 0.00032 & 0.00031 & 0.00031 \\
\hline $2 \mathrm{M}$ & 0.00037 & 0.00025 & 0.00023 & 0.00023 & 0.00023 \\
\hline \multicolumn{6}{|c|}{ Unified Cache } \\
\hline \begin{tabular}{|l|} 
Size \\
\end{tabular} & Direct & 2-way & 4-way & 8-way & 16-way \\
\hline $1 \mathrm{~K}$ & 0.26420 & 0.15450 & & & \\
\hline $2 \mathrm{~K}$ & 0.18713 & 0.10727 & 0.09447 & & \\
\hline $4 \mathrm{~K}$ & 0.13043 & 0.07159 & 0.06196 & 0.05769 & \\
\hline $8 \mathrm{~K}$ & 0.07065 & 0.04580 & 0.03806 & 0.03514 & 0.03401 \\
\hline $16 \mathrm{~K}$ & 0.04156 & 0.02468 & 0.02112 & 0.01939 & 0.01851 \\
\hline $32 \mathrm{~K}$ & 0.02763 & 0.01314 & 0.01030 & 0.00941 & 0.00900 \\
\hline $64 \mathrm{~K}$ & 0.01455 & 0.00672 & 0.00474 & 0.00414 & 0.00384 \\
\hline $128 \mathrm{~K}$ & 0.00730 & 0.00324 & 0.00232 & 0.00205 & 0.00198 \\
\hline $256 \mathrm{~K}$ & 0.00325 & 0.00156 & 0.00130 & 0.00122 & 0.00121 \\
\hline $512 \mathrm{~K}$ & 0.00145 & 0.00053 & 0.00045 & 0.00043 & 0.00041 \\
\hline $1 \mathrm{M}$ & 0.00055 & 0.00013 & 0.00011 & 0.00010 & 0.00010 \\
\hline $2 \mathrm{M}$ & 0.00041 & 0.00008 & 0.00007 & 0.00007 & 0.00007 \\
\hline
\end{tabular}

Block Size: 256 bytes

\begin{tabular}{|c|c|c|c|c|c|}
\hline \multicolumn{6}{|c|}{ SPECfP95 } \\
\hline \multirow{2}{*}{\multicolumn{6}{|c|}{$\begin{array}{c}\text { Instruction Cache } \\
\text { Associativity }\end{array}$}} \\
\hline & & & & & \\
\hline Size & Direct & 2-way & 4-way & 8-way & 16-way \\
\hline $1 \mathrm{~K}$ & 0.01072 & 0.01097 & & & \\
\hline $2 \mathrm{~K}$ & 0.00663 & 0.00639 & 0.00701 & & \\
\hline $4 \mathrm{~K}$ & 0.00356 & 0.00333 & 0.00315 & 0.00319 & \\
\hline $8 \mathrm{~K}$ & 0.00229 & 0.00194 & 0.00190 & 0.0 & 0.00200 \\
\hline $16 \mathrm{~K}$ & 0.00171 & 0.00116 & 0.00107 & 0.00107 & 0.00105 \\
\hline $32 \mathrm{~K}$ & 0.00068 & 0.00044 & 0.00047 & 0.00076 & 0.00089 \\
\hline $64 \mathrm{~K}$ & 0.00009 & 0.00004 & 0.00000 & 0.00000 & 0.00000 \\
\hline $128 \mathrm{~K}$ & 0.00005 & 0.00000 & 0.00000 & 0.00000 & 0.00000 \\
\hline $256 \mathrm{~K}$ & 0.00001 & 0.00000 & 0.00000 & 0.00000 & 0.00000 \\
\hline $512 \mathrm{~K}$ & 0.00001 & 0.00000 & 0.00000 & 0.00000 & 0.00000 \\
\hline $1 \mathrm{M}$ & 0.00000 & 0.00000 & 0.00000 & 0.00000 & 0.00000 \\
\hline $2 \mathrm{M}$ & 0.0 & 0.00000 & 0.00000 & 0.00000 & 0.00000 \\
\hline \multicolumn{6}{|c|}{ Data Cache } \\
\hline Size & Direct & 2-way & 4-way & 8-way & 16-way \\
\hline $1 \mathrm{~K}$ & 0.46511 & 0.41613 & & & \\
\hline $2 \mathrm{~K}$ & 0.34652 & 0.27972 & 0.23807 & & \\
\hline $4 \mathrm{~K}$ & 0.25468 & 0.19641 & 0.14355 & 0.10942 & \\
\hline $8 \mathrm{~K}$ & 0.18700 & 0.14094 & 0.10050 & 0.06468 & 0.02253 \\
\hline $16 \mathrm{~K}$ & 0.13548 & 0.10278 & 0.08283 & 0.05667 & 0.01651 \\
\hline $32 \mathrm{~K}$ & 0.09046 & 0.06506 & 0.03537 & 0.03172 & 0.01164 \\
\hline $64 \mathrm{~K}$ & 0.04385 & 0.03898 & 0.01515 & 0.01146 & 0.01000 \\
\hline $128 \mathrm{~K}$ & 0.01881 & 0.01254 & 0.01247 & 0.00829 & 0.00841 \\
\hline $256 \mathrm{~K}$ & 0.01183 & 0.00826 & 0.00828 & 0.00748 & 0.00760 \\
\hline $512 \mathrm{~K}$ & 0.00767 & 0.00615 & 0.00487 & 0.00490 & 0.00496 \\
\hline $1 \mathrm{M}$ & 0.00605 & 0.00461 & 0.00436 & 0.00431 & 0.00432 \\
\hline $2 \mathrm{M}$ & 0.00438 & 0.00356 & 0.00351 & 0.00354 & 0.00357 \\
\hline \multicolumn{6}{|c|}{ Unified Cache } \\
\hline Size & Direct & 2-way & 4-way & 8-way & 16-way \\
\hline $1 \mathrm{~K}$ & 0.29513 & 0.18652 & & & \\
\hline $2 \mathrm{~K}$ & 0.19442 & 0.1 & 0.10607 & & \\
\hline $4 \mathrm{~K}$ & 0.13132 & 0.0 & 0.06271 & 0.05270 & \\
\hline $8 \mathrm{~K}$ & 0.08645 & 0.0 & 0.03934 & 0.02708 & 0.01299 \\
\hline $16 \mathrm{~K}$ & 0.05718 & 0.03 & 0.02982 & 0.02145 & 0.00766 \\
\hline $32 \mathrm{~K}$ & 0.03896 & 0.02252 & 0.01311 & 0.01181 & 0.00505 \\
\hline $64 \mathrm{~K}$ & 0.01970 & 0.01314 & 0.00541 & 0.00407 & 0.00354 \\
\hline $128 \mathrm{~K}$ & 0.00847 & 0.00423 & 0.00410 & 0.00274 & 0.00277 \\
\hline $56 \mathrm{~K}$ & 0.00558 & 0.00269 & 0.00268 & 0.00244 & 0.00247 \\
\hline $512 \mathrm{~K}$ & 0.00324 & 0.00201 & 0.00161 & 0.00162 & 0.00165 \\
\hline $1 \mathrm{M}$ & 0.00259 & 0.0 & 0.00143 & 0.00141 & 0.00141 \\
\hline $2 \mathrm{M}$ & 0.002 & 0.00117 & 0.00115 & 0.001 & 0.00 \\
\hline
\end{tabular}

Table 15: Arithmetic Average Miss Ratios for SPEC95 (256 byte Block Size) 